\title{
Role of Notch Signaling in Pulmonary Hypertension
}

\author{
Inaugural Dissertation \\ submitted to the \\ Faculty of Veterinary Medicine or Faculty of Medicine \\ in partial fulfillment of the requirements \\ for the PhD-Degree \\ of the Faculties of Veterinary Medicine and Medicine \\ of the Justus Liebig University Giessen
}

\author{
by \\ Dabral, Swati \\ of \\ Kotdwara, India
}

Giessen (2012) 
From the Department of Internal Medicine

Director/Chairman: Prof. Dr. med. Werner Seeger of the University Hospital Giessen - Marburg

First Supervisor and Committee Member: Prof. Ralph Theo Schermuly, PhD

Committee Member: PD. Dr. Hanno Leuchte

Date of Doctoral Defense: 29.06.13 


\section{TABLE OF CONTENTS}

TABLE OF CONTENTS

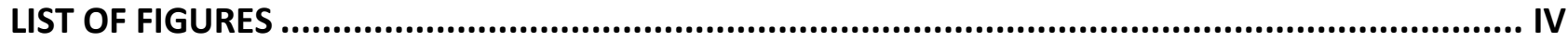

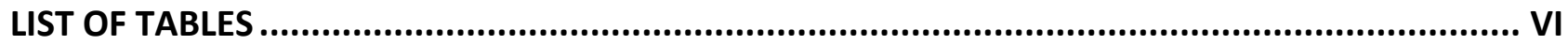

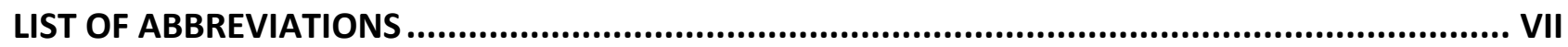

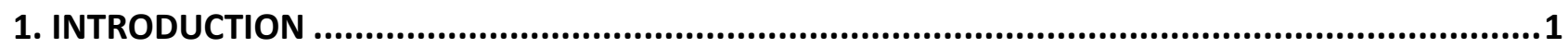

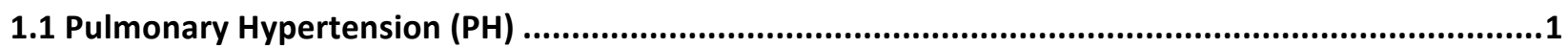

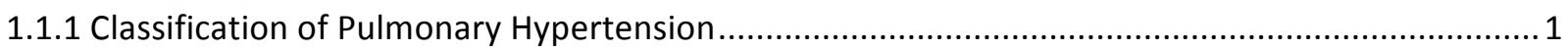

1.1.2 Patho-physiology of Pulmonary Arterial Hypertension (PAH) ..................................................

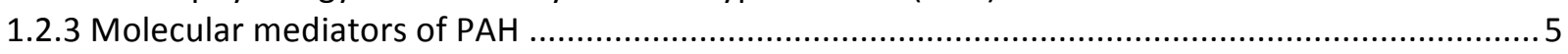

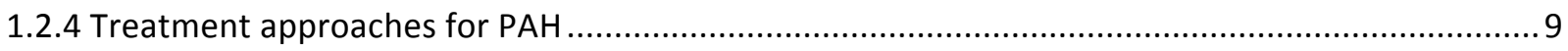

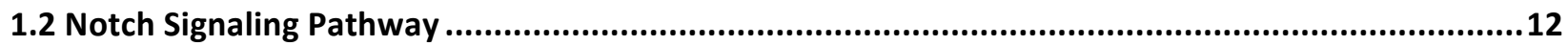

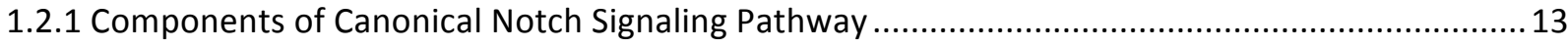

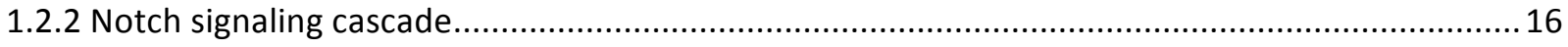

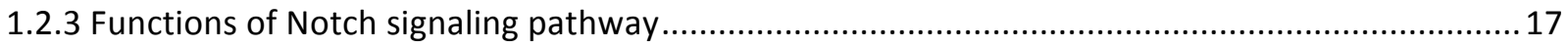

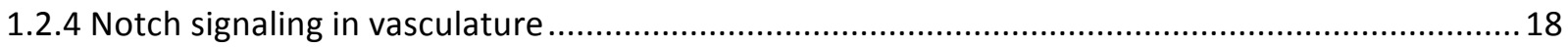

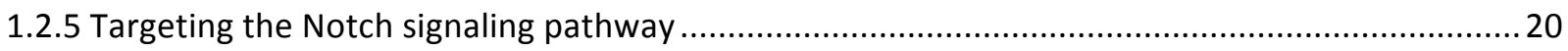

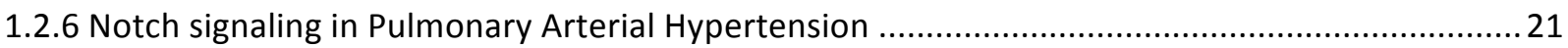

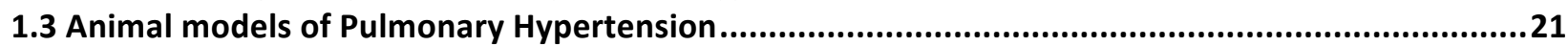

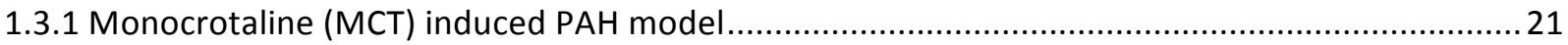

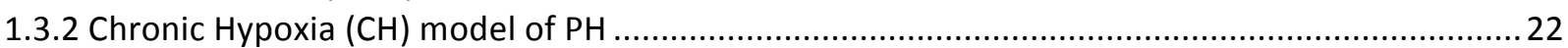

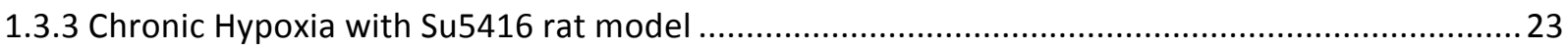

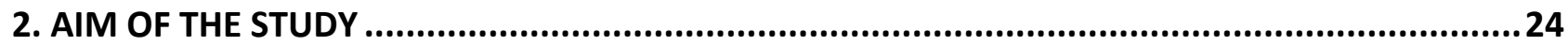

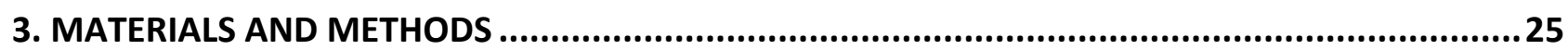

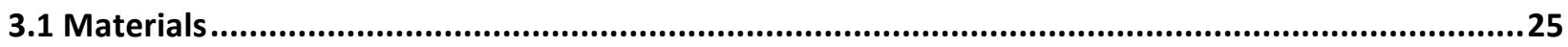

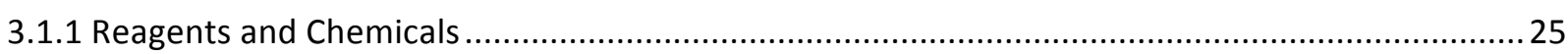

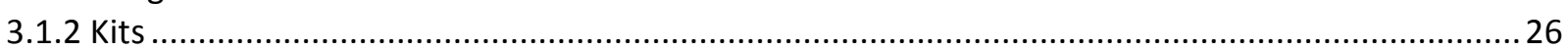

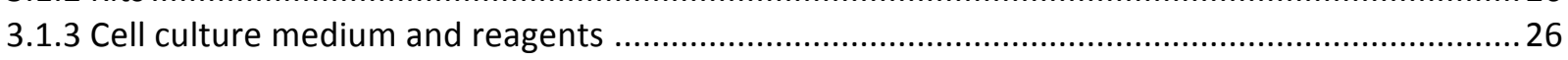

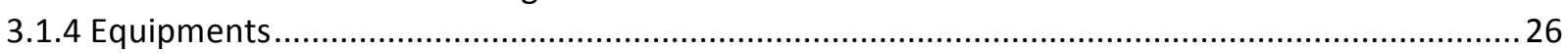

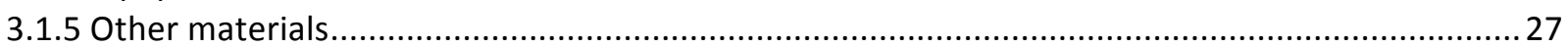

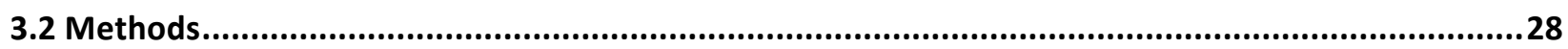

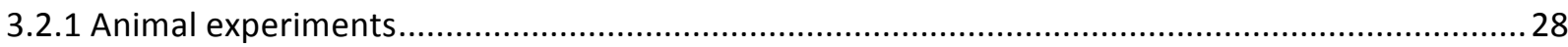

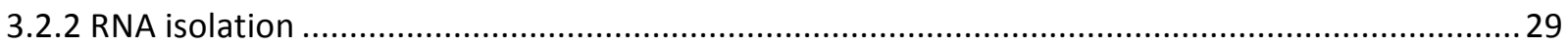

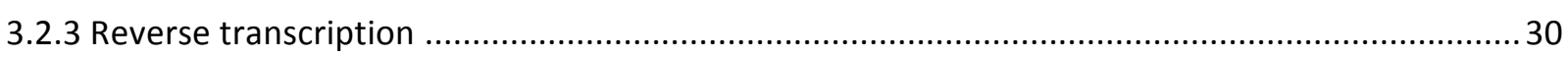

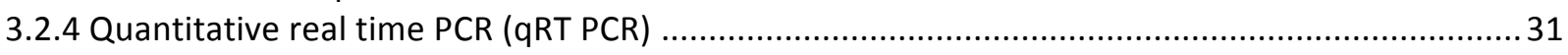

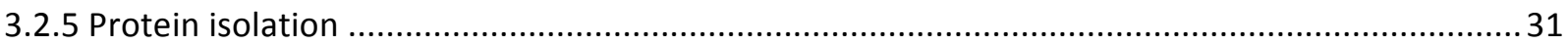

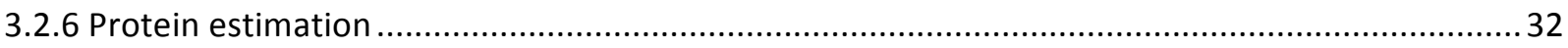

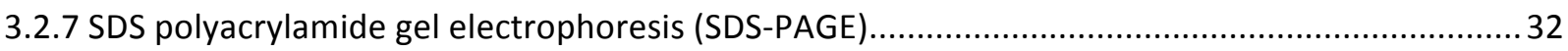

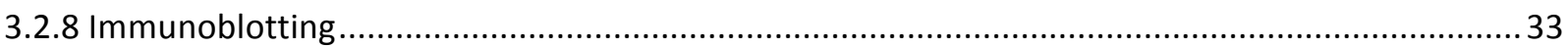

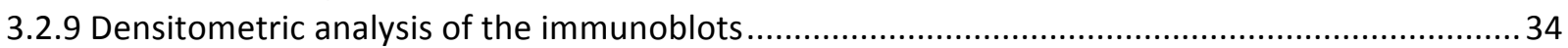

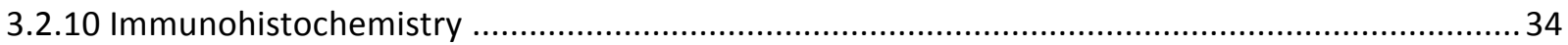

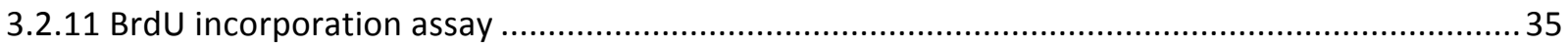

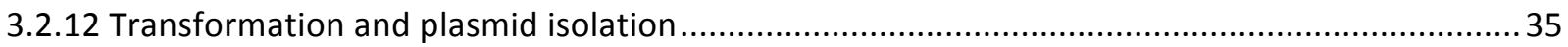

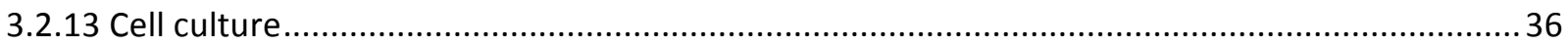

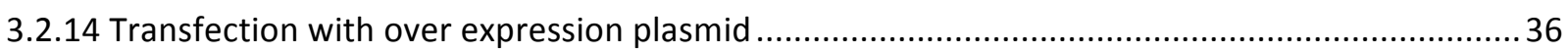

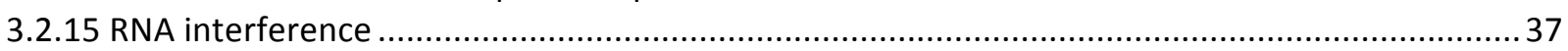

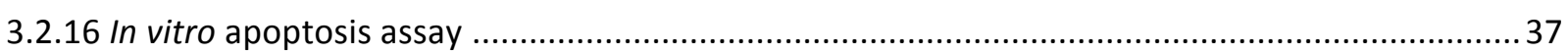




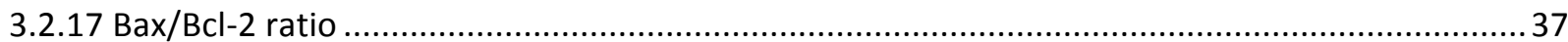

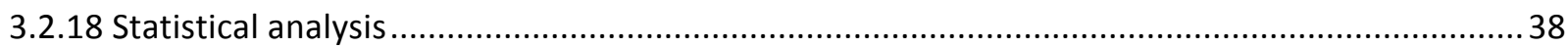

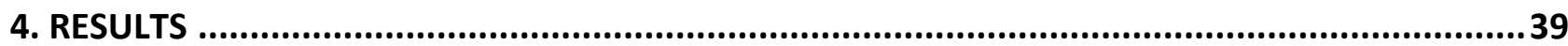

4.1 Regulation of Notch receptors and ligands in monocrotaline (MCT) induced $\mathrm{PH}$ rats ...................39

4.1.1 Hemodynamics and right heart hypertrophy in MCT rats ......................................................39

4.1.2 mRNA expression of Notch receptors and ligands in lung homogenates..................................39

4.1.3 Protein expression of Notch receptors and ligands in lung homogenates .................................40

4.2 Regulation of Notch receptors and ligands in hypoxia (Hox) induced PH mice ...........................41

4.2.1 Hemodynamics and right heart hypertrophy in Hox mice .............................................41

4.2.2 mRNA expression of Notch receptors and ligands in lung homogenates.................................42

4.2.4 Protein expression of Notch receptors and ligands in lung homogenates ................................43

4.3 Regulation of Notch receptors and ligands in Hypoxia+Su5416 (Hox+Su5416) induced PAH rats....43

4.3.1 Hemodynamics and right heart hypertrophy in Hox+Su5416 rats ......................................43

4.3.2 mRNA expression of Notch receptors and ligands in lung homogenates.................................44

4.3.3 Protein expression of Notch receptors and ligands in lung homogenates .............................44

4.4 Regulation of Notch receptors and ligands in IPAH patients..............................................45

4.4.1 mRNA expression of Notch receptors and ligands in lung homogenates................................45

4.4.2 Protein expression of Notch receptors and ligands in lung homogenates ..............................45

4.4.3 mRNA and protein expression of Notch1 in pulmonary arteries ..........................................46

4.3 Localization of Notch1 in IPAH and donor lungs ..............................................................47

4.5 Effect of Notch1 knockdown on cellular proliferation and apoptosis in hPAECs ...........................48

4.5.1 Transient Notch1 knockdown by siRNA ...........................................................................49

4.5.2 Notch1 knockdown attenuates hPAECs proliferation .........................................................50

4.5.3 Inhibition of Notch1 modulates cell cycle markers ..........................................................50

4.5.4 Notch1 knockdown promotes apoptosis in hPAECs ...........................................................5

4.6 Effect of Notch1ICD over expression on cellular proliferation and apoptosis in human pulmonary

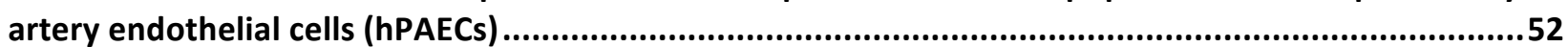

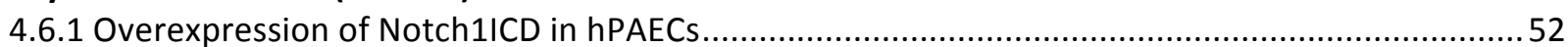

4.6.2 Notch1 ICD overexpression increases proliferation of hPAECs .................................................53

4.6.3 Notch1 ICD over-expression modulates cell cycle markers ..................................................53

4.6.4 Notch1 mediates anti-apoptotic effect on hPAECs .........................................................5

4.7 Effect of Notch1 knock down and over-expression on proliferation of human pulmonary artery smooth muscle cells (hPASMCs) .....................................................................................55

4.7.1 Notch1 knock down has no effect on proliferation of hPASMCs ............................................55

4.7.2 Notch1 over-expression has no effect on proliferation of hPASMCs .......................................56

4.8 Effect of pharmacological inhibition of Notch signaling using $\boldsymbol{\gamma}$-secretase inhibitor, DBZ on hPAECs and hPASMCs

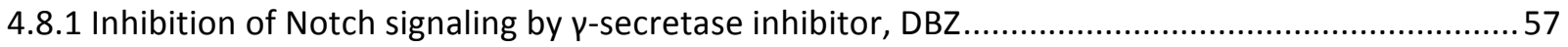

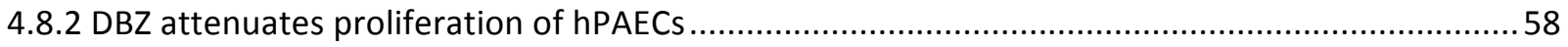

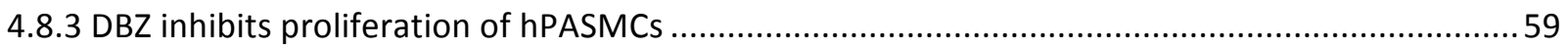

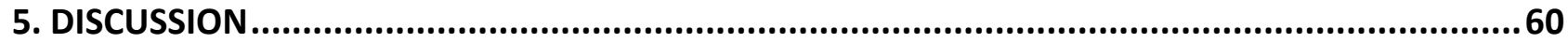

5.1 Expression of Notch receptors and ligands in IPAH patients.................................................60

5.2 Expression of Notch receptors and ligands in experimental PH models.....................................61

5.3 Localization of Notch1 in IPAH lungs.....................................................................63

5.4 Influence of Notch1 on proliferation of hPAECs ..........................................................63

5.5 Effect of Notch1 on survival of hPAECs ............................................................................65

5.6 Effect of Notch1 on proliferation of hPASMCs ...............................................................66

5.7 Pharmacological inhibition of Notch signaling inhibits hPASMCs and hPAECs proliferation............66

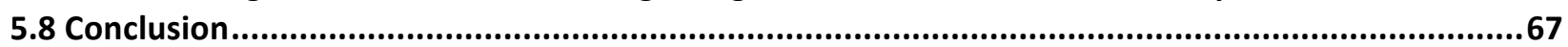

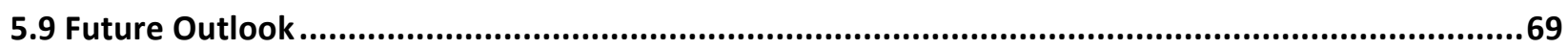




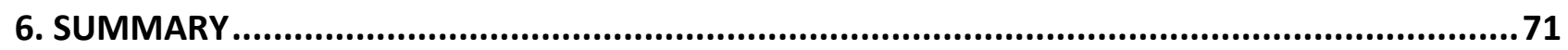

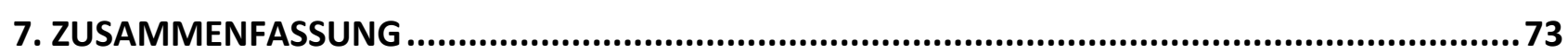

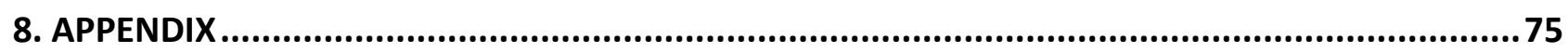

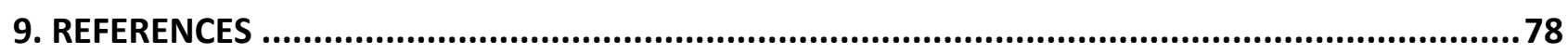

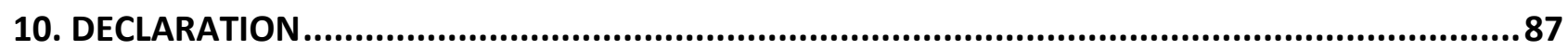

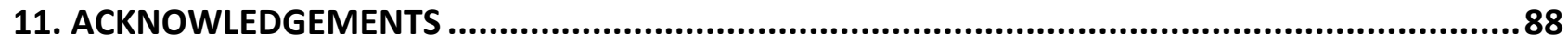

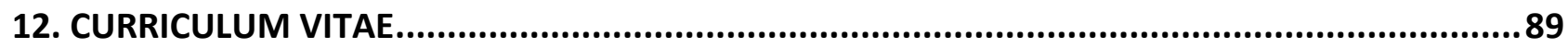




\section{LIST OF FIGURES}

Figure 1.1 Histology of PAH.

Figure 1.2 Current and emerging targets and therapies for PAH.

Figure 1.3 Schematic representation of general domain organization of Notch pathway receptors in mammals.

Figure 1.4 Schematic representation of general domain organization of DSL ligands in mammals.

Figure 1.5 Domain organisation of Hes and Hey proteins.

Figure 1.6 Schematic representation of canonical Notch Signaling pathway.

Figure 1.7 Pleiotropic effects of Notch Signaling.

Figure 1.8 Schematic representation of putative therapeutic targets in Notch signaling.

Figure 1.9 Pulmonary artery remodeling in animal models compared to IPAH.

Figure 4.1 Hemodynamic measurements and right heart hypertrophy in Monocrotaline induced $\mathrm{PH}$ rats.

Figure 4.2 mRNA expression of Notch receptors and ligands in lung homogenates of MCT induced $\mathrm{PH}$ rats.

Figure 4.3 Protein expression of Notch receptors and ligands in lung homogenates of MCT induced $\mathrm{PH}$ rats.

Figure 4.4 Hemodynamic measurements and right heart hypertrophy in Hypoxia (Hox) induced $\mathrm{PH}$ mice.

Figure 4.5 Expression of Notch receptors and ligands in lung homogenates of Hox induced PH mice.

Figure 4.6 Hemodynamic measurements and right heart hypertrophy in Hypoxia+Su5416 (3wHox+Su5416) induced $\mathrm{PH}$ rats.

Figure 4.7 Expression of Notch receptors and ligands in Hypoxia+Su5416 rat lungs.

Figure 4.8 Expression of Notch receptors and ligands in human donors and IPAH lungs.

Figure 4.9 Expression of Notch1 in human donors and IPAH pulmonary arteries.

Figure 4.10 Immunolocalization of Notch1 in lungs of IPAH patients.

Figure 4.11 Knockdown of Notch1 in hPAECs.

Figure 4.12 Notch1 knockdown attenuates proliferation of hPAECs.

Figure 4.13 Notch1 knockdown induces apoptosis in hPAECs. 
Figure 4.14 Overexpression of Notch1 Intracellular domain (N1ICD) in hPAECs.

Figure 4.15 N1ICD overexpression increases proliferation of hPAECs.

Figure 4.16 N1ICD overexpression promotes survival of hPAECs.

Figure 4.17 Effect of Notch1 knockdown on proliferation of hPASMCs.

Figure 4.18 Effect of N1ICD overexpression on proliferation of hPASMCs.

Figure 4.19 $\quad y$-secretase inhibitor (DBZ) inhibits Notch signaling in hPASMCs and hPAECs.

Figure 4.20 $\quad y$-secretase inhibitor (DBZ) inhibits Notch signaling in hPAECs.

Figure 4.21 $\quad \gamma$-secretase inhibitor (DBZ) inhibits Notch signaling in hPASMCs.

Figure 5.1 Schematic representation of proposed role of Notch1 in endothelial proliferation and survival in $\mathrm{PH}$. 


\section{LIST OF TABLES}

Table 1. Updated Classification of PH

Table 1 App. List of primers used for real time PCR

Table 2 App. List of primary antibodies

Table 3 App. List of secondary antibodies 


\section{LIST OF ABBREVIATIONS}

$\begin{array}{ll}\text { 5-HT } & \text { 5-Hydroxytryptamine, Serotonin } \\ \text { 5-HTT } & \text { 5-Hydroxytryptamine Transporter } \\ \text { BAX } & \text { Bcl2 associated X protein } \\ \text { Bcl-2 } & \text { B cell lymphoma 2 } \\ \text { bHLH } & \text { basic helix loop helix } \\ \text { BrdU } & \text { Bromodeoxyuridine } \\ \text { BMPRII } & \text { Bone Morphogenetic Protein 2 } \\ \text { BSA } & \text { Bovine Serum Albumin } \\ \text { Ca }^{2+} & \text { Calcium }\end{array}$

CADASIL Cerebral Autosomal Dominant Arteriopathy with Subcortical Infarcts and Leukoencephalopathy

cDNA single stranded DNA

CGMP cyclic guanosine monophosphate

CCL2 Chemokine receptor 2

$\mathrm{CH} \quad$ Chronic Hypoxia

CML Chronic MyelogenousLeukemia

CSL $\quad$ CBF1, Su(H) and Lag1

CTEPH Chronic Thromboembolic Pulmonary Hypertension

CX3CR1 Chemokine receptor 1

DBZ Dibenzapine (gamma-secretase inhibitor)

DDAH2 Dimethylarginine dimethyl aminohydrolase 2

DLL Delta like Ligand

DPBS Dulbecco's Phosphate Buffer Saline

DNA Deoxyribo Nucleic Acid

DSL Delta Serrate Lag-2

EC Endothelial cell

ECM Extracellular Matrix

EDTA Ethylenediaminetetraacetic acid

EGF Epidermal Growth Factor

eNOS endothelial Nitric Oxide Synthase

ER Endoplasmic Reticulum

ET-1 Endothelin 1

ETRA Endothelin Receptor A

ETRB Endothelin Receptor B

FCS Fetal Calf Serum

FGF2 Fibroblast Growth Factor 2

GAPDH Glycelraldehyde 3-phosphate dehydrogenase

GSI gamma-secretase inhibitor

HES Hairy/enhancer of split

HERP Hes related-repressor protein 


\begin{tabular}{|c|c|}
\hline HIF-1 $\alpha$ & Hypoxia Inducible Factor $1 \alpha$ \\
\hline Hox & Hypoxia \\
\hline hPAEC & human Pulmonary Artery Endothelial Cell \\
\hline hPASMC & human Pulmonary Artery Smooth Muscle Cell \\
\hline HPV & Hypoxic Pulmonary Vasoconstriction \\
\hline HRP & Horseradish Peroxidase \\
\hline IAP & Inhibitor of Apoptosis \\
\hline ICD & Intracellular domain \\
\hline IGF & Insulin-like Growth Factor \\
\hline IgG & Immunoglobin \\
\hline IL-6 & Interlukin-6 \\
\hline IPAH & Idiopathic Pulmonary Arterial Hypertension \\
\hline KO & Knock Out \\
\hline Kv channel & Potassium channel \\
\hline LNRs & Lin-12 notch repeats \\
\hline MCT & Monocrotaline \\
\hline $\mathrm{MgCl}_{2}$ & Magnesium Chloride \\
\hline mRNA & messenger Ribonucleic Acid \\
\hline N1ICD & Notch1 Intracellular Domain \\
\hline NEXT & Notch Extracellular Truncation fragment \\
\hline NO & Nitric Oxide \\
\hline NOS & Nitric Oxide Synthase \\
\hline PA & Pulmonary Artery \\
\hline PAH & Pulmonary Arterial Hypertension \\
\hline PAP & Pulmonary Arterial Pressure \\
\hline PAEC & Pulmonary Artery Endothelial Cell \\
\hline PAFBs & Pulmonary Artery Adventitial Fibroblasts \\
\hline PASMC & Pulmonary Artery Smooth Muscle Cell \\
\hline PBGD & Phorphobilinogen Deaminase \\
\hline PDE-5 & Phosphodiesterae-5 \\
\hline PDGF & Platelet Derived Growth Factor \\
\hline PDGFR $\beta$ & Platelet Derived Growth Factor $\beta$ \\
\hline PGI2 & Prostacyclin \\
\hline PPH & Primary Pulmonary Hypertension \\
\hline PVR & Pulmonary Vascular Resistance \\
\hline RBJK & Recombination signal sequence-Binding Protein JK \\
\hline RIPA & Radioimmunoprecipitation Assay \\
\hline RNA & Ribonucleic Acid \\
\hline RT & Reverse Transcriptase \\
\hline RVSP & Right Ventricular Systolic Pressure \\
\hline SAP & Systemic Arterial Pressure \\
\hline
\end{tabular}


SU5416 SUGEN (Vascular Endothelial Growth Factor receptor-2 inhibitor)

SYBR Green asymmetrical cyanine dye used as a nucleic acid stain in molecular biology

TAE Tris base, acetic acid and EDTA buffer

TBS Tris Buffered Saline

TBST Tris Buffered Saline + Tween 20

TGF $\beta \quad$ Transforming Growth Factor $\beta$

TNF Tumor Necrosis Factor

TUNEL Terminal deoxynucleotidyltransferasedUTP nick end labeling

VEGF Vascular Endothelial Growth Factor

VEGFR2 Vascular Endothelial Growth Factor receptor 2

VIP Vasoactive Intestinal Peptide

vWF von Willebrand Factor 


\section{INTRODUCTION}

\subsection{Pulmonary Hypertension (PH)}

Pulmonary Hypertension $(\mathrm{PH})$ is a progressive disease characterized by increased pulmonary vascular resistance (PVR) leading to right heart hypertrophy and ultimately death of the patient due to right heart failure [1]. Generally, pulmonary circulation is a low pressure and high flow system, maintaining pressure of about $15 \mathrm{~mm} \mathrm{Hg}$; however, in $\mathrm{PH}$ due to increased pulmonary vascular resistance, the pressures reach up to $25 \mathrm{~mm} \mathrm{Hg}$ at rest and $30 \mathrm{~mm} \mathrm{Hg}$ during exercise [2]. In all its different forms, $\mathrm{PH}$ is estimated to effect 100 million people worldwide, with the median survival rate of 2.8 years after diagnosis [3].

\subsubsection{Classification of Pulmonary Hypertension}

Dresdale and colleagues first reported a hemodynamic account of IPAH in 1951[4]. The first classification of $\mathrm{PH}$ was carried out in 1973 at an international conference on primary $\mathrm{PH}$, dividing the patients into two categories, primary $\mathrm{PH}$ and secondary $\mathrm{PH}$, depending on absence or presence of recognizable causes [5]. A new classification of $\mathrm{PH}$ was introduced in 1998 , at the $2^{\text {nd }}$ World Symposium on Pulmonary Arterial Hypertension held in Evian, France. The "Evian classification" attempted to create categories of $\mathrm{PH}$ on the basis of shared pathologic and clinical features as well as similar therapeutic options. This was a much broader, more precise classification, with 5 major categories, allowing investigators to conduct clinical trials in a welldefined group of patients with a shared underlying pathogenesis [1]. Since then, the classification has undergone some revisions, owing to newfound knowledge about the disease. A major change was proposed in the classification at $3^{\text {rd }}$ World Conference on PH in 2003. In this classification, PPH was replaced with idiopathic PAH (IPAH) or, when supported by genetic basis, familial PAH (FPAH) [6]. Current classification, agreed upon in $4^{\text {th }}$ World Symposium on Pulmonary Hypertension in 2008 at Dana Point, still maintains the structure of Evian classification with minor adjustments (Table 1). 


\section{Pulmonary arterial hypertension (PAH)}

1.1 Idiopathic $\mathrm{PAH}$

1.2 Heritable

1.2.1 BMPR2

1.2.2 ALK1, endoglin (with or without hereditary hemorrhagic telangiectasia)

1.2.3 Unknown

1.3 Drug- and toxin-induced

1.4 Associated with

1.4.1 Connective tissue diseases

1.4.2 HIV infection

1.4.3 Portal hypertension

1.4.4 Congenital heart diseases

1.4.5 Schistosomiasis

1.4.6 Chronic hemolytic anemia

1.5 Persistent pulmonary hypertension of the newborn

1' Pulmonary veno-occlusive disease (PVOD) and/or pulmonary capillary hemangiomatosis (PCH)

\section{Pulmonary hypertension owing to left heart disease}

2.1 Systolic dysfunction

2.2 Diastolic dysfunction

2.3 Valvular disease

\section{Pulmonary hypertension owing to lung diseases and/or hypoxia}

3.1 Chronic obstructive pulmonary disease

3.2 Interstitial lung disease

3.3 Other pulmonary diseases with mixed restrictive and obstructive pattern

3.4 Sleep-disordered breathing

3.5 Alveolar hypoventilation disorders

3.6 Chronic exposure to high altitude

3.7 Developmental abnormalities

\section{Chronic thromboembolic pulmonary hypertension (CTEPH)}

\section{Pulmonary hypertension with unclear multifactorial mechanisms}

5.1 Hematologic disorders: myeloproliferative disorders, splenectomy

5.2 Systemic disorders: sarcoidosis, pulmonary Langerhans cell histiocytosislymphangioleiomyomatosis, neurofibromatosis, vasculitis

5.3 Metabolic disorders: glycogen storage disease, Gaucher disease, thyroid disorders

5.4 Others: tumoral obstruction, fibrosingmediastinitis, chronic renal failure on dialysis

Table 1. Updated Classification of PH (Dana Point, 2008) [6] ALK1, activin receptor-like kinase type 1; BMPR2, bone morphogenetic protein receptor type 2; HIV, human immunodeficiency virus

\subsubsection{Patho-physiology of Pulmonary Arterial Hypertension (PAH)}

PAH has a multifactorial pathobiology. However, regardless of the initial trigger, the increased pulmonary artery pressure and vascular resistance observed in patients suffering from $\mathrm{PAH}$, are an outcome of remodeling and in-situ thrombosis of small- and medium-sized pulmonary 
arteries and arterioles, as well as sustained vasoconstriction [7].

\subsubsection{Pulmonary vasoconstriction}

Vasoconstriction defined as narrowing of lumen of the blood vessel is a major contributor to PVR, and, hence, elevated pulmonary artery pressure (PAP) [8]. Under normal conditions, pulmonary vascular tone is maintained by balancing actions of endothelium derived vasodilators, mainly prostacyclin (PGI2), nitric oxide (NO) and vasoconstrictors, namely thromboxane A2 and endothelin-1(ET-1) [9]. Endothelial dysfunction caused due to shear stress or inflammation, can disturb this balance contributing to persistent vasoconstriction. Patients with PAH have been shown to have reduced circulating levels of vasodilator, prostacyclin as compared to levels of vasoconstrictor, thromboxane [10]. Hypoxia mediated pulmonary vasoconstriction, an adaptive mechanism unique to lungs, is also believed to be a major factor behind high altitude-PH [8].

\subsubsection{In-situ thrombosis}

One of the major histological representations of $\mathrm{PH}$ is in situ thrombosis of pulmonary arterioles [11]. Endothelial dysfunction, abnormalities in clotting cascade and platelet activation, creating a pro-coagulant environment within the pulmonary vascular bed are believed to be responsible for this pathological finding $[7,8]$. Increased plasma levels of fibrinopeptide1, a marker for fibrin generation and plasminogen activator inhibitor1, inhibitor of fibrinolysis have been reported in IPAH patients [12]. Von Willebrand factor (VWF), essential for interaction of endothelial cells with platelets has been shown to have increased activity in patients with IPAH [12]. Imbalance in vasoactive mediators: increase in proaggregatory thromboxane A2 and decrease in aggregation inhibiting prostaglandins and NO observed in PAH patients, further results in heightened platelet aggregation and enhanced thrombosis in pulmonary vasculature [13]. Though thrombotic lesions in pulmonary vasculature are found in all severe forms of $\mathrm{PH}$, these lesions have never been associated with the severity of disease [14].

\subsubsection{Vascular remodeling}

Vascular remodeling refers to structural changes that result in hypertrophy and/or luminal occlusions. A fine balance between proliferation and apoptosis of fibroblasts, pulmonary artery smooth muscle cells (PASMCs) and -endothelial cells (PAECs) are maintained under normal conditions. Disturbing this balance in favour of proliferation as observed in $\mathrm{PH}$, results in thickening of pulmonary arterial wall and eventually, obliteration of the vessel lumen leading to 
increased PVR [8]. In PAH, the common vascular histological findings involve remodeling of all three layers characterized by intimal hyperplasia and plexiform lesions, medial hypertrophy and adventitial proliferation [2] (Figure 1.1).
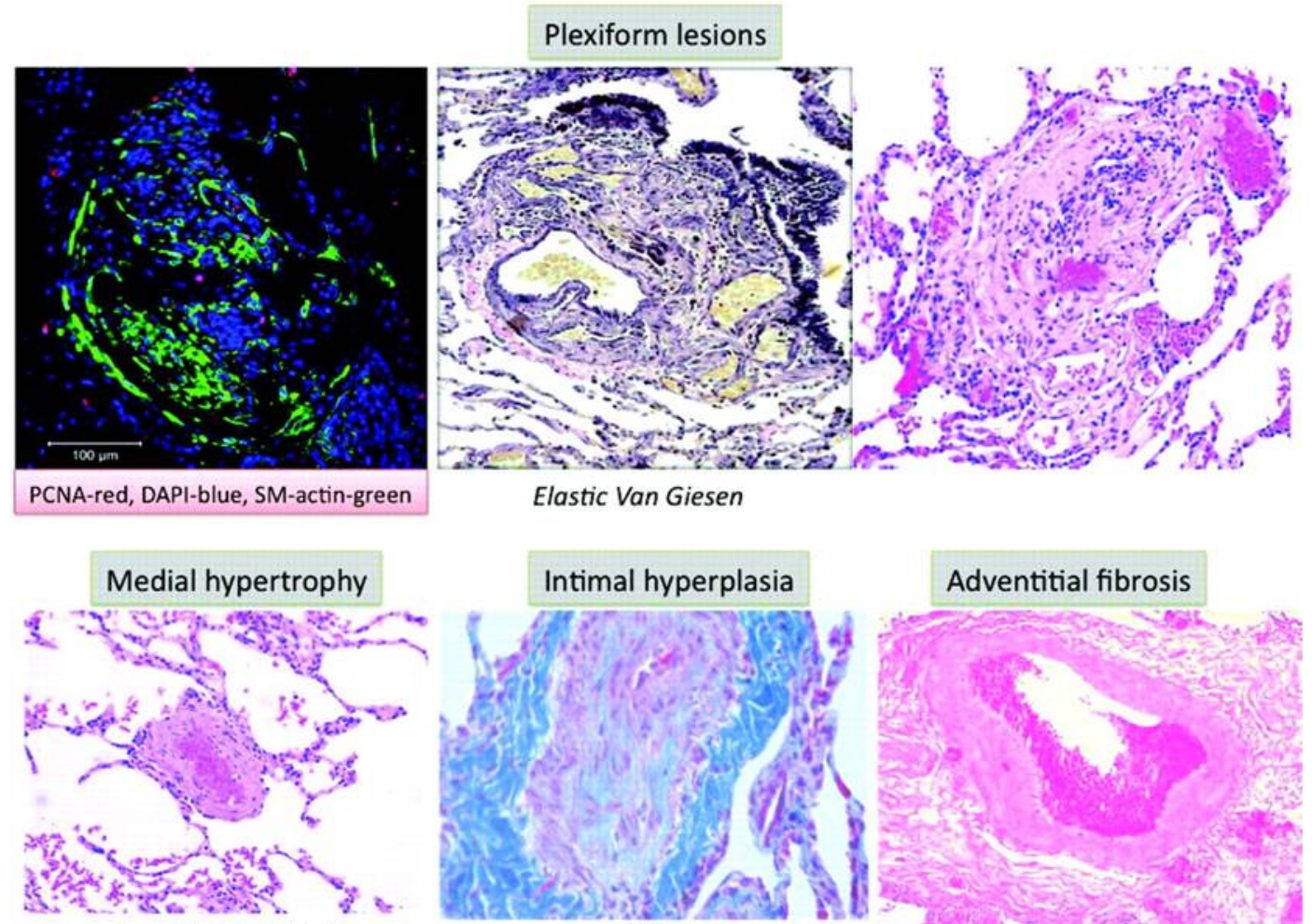

Elastic Van Giesen

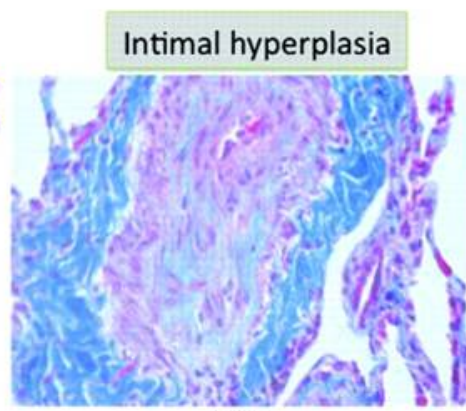

Trichrome
Adventitial fibrosis

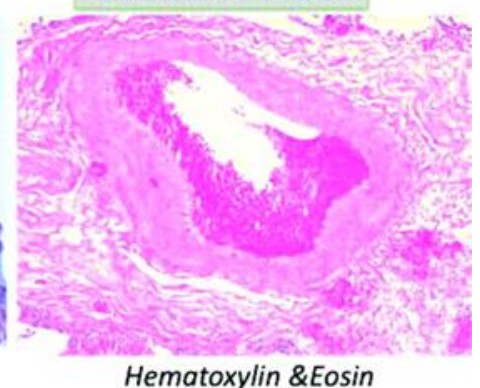

Figure 1.1 Histology of PAH. Top: Plexiform lesions. Upper Left: Evidence of cell proliferation (red is proliferating cell nuclear antigen [PCNA], green is smooth muscle [SM] actin, and blue is DAPI). Bottom: Medial hypertrophy, intimal fibrosis, and adventitial proliferation. (Archer et.al, Circulation, 2010) [15]

\section{Intimal lesions}

Intimal lesions account for major reduction in luminal area of small pulmonary arteries and hence, largely influence the overall PVR. These lesions consist of eccentric intima thickening and concentric and fibrotic plexiform lesions [16].

Intima thickening can be concentric laminar, eccentric, or concentric nonlaminar. Cells present in the thickening exhibit properties of fibroblasts, myofibroblasts and smooth muscle cells $[16,17]$. In severe cases, lesions are characterized by "fibrotic" pattern, with abundant extracellular matrix (ECM) deposition.

Plexiform lesions, generally observed at branch points, are complex and disorganized pulmonary arterial proliferative lesion consisting of a network or plexus of channels lined by endothelial cells supported by stroma containing matrix proteins and myofibroblasts [7, 18]. 


\section{Medial hypertrophy}

A prominent feature of vascular remodeling is medial smooth muscle cell hypertrophy involving muscularized arteries (70-800 $\mu \mathrm{m}$ in diameter) and pre-capillary vessels (below $70 \mu \mathrm{m}$ in diameter) [16]. In previously muscularized vessels, medial thickening is caused by hypertrophy and hyperplasia of the existing smooth muscle cells (SMCs) [19]. Increased proliferation of the cells is accompanied with a decreased rate of apoptosis. Additionally, SMCs and fibroblasts produce extracellular matrix proteins which further contribute to remodeling [16]. In pre-capillary (nonmuscularized) vessels, de novo formation of muscular media is observed. Smooth muscle cells, which form the new media, are suggested to be derived from either differentiation of intermediate cells present in the vessels or from migration and differentiation of adventitial fibroblasts $[19,20]$.

\section{Adventitial proliferation}

Adventitial layer, mostly composed of fibroblasts, initially considered a mere structural support for vessels has come up in recent research as an important player in pulmonary vascular remodeling. In animal models of hypoxia, the earliest and most dramatic structural changes are observed in the adventitial layer of the vessel wall, which further initiate remodeling of the medial and intimal compartments [21]. It has been established that the heterogenous population of pulmonary artery adventitial fibroblasts (PAFBs), can proliferate and secrete various chemokines facilitating recruitment of inflammatory cells and differentiate into myofibroblasts in response to environmental stress [20]. Myofibroblasts are the major producers of collagen and other ECM proteins and can further, migrate to medial or intimal layer effecting neointima formation. Accumulation of myofibroblasts in intima of patients with PAH is well documented [18].

\subsubsection{Molecular mediators of PAH}

With the growing amount of research, perturbations in a number of molecular mechanisms have been described as the players in pathogenesis of PAH. Various changes in signaling pathways involving vasoconstriction, growth factors and inflammation have shown to drive the disease progression.

\subsubsection{Vascular tone modulators and enhancers}

\section{Prostacyclin}

Prostacyclin (PGI2), a product of arachidonate metabolism by cyclooxygenase, is a strong endothelium derived vasodilator. Additionally, it exerts an anti-proliferative effect on vascular 
smooth muscle cells and anti-aggregation effect on platelets[22]. Prostacyclin production is decreased in endothelial cells from PAH patients [10], attributable to suppression of prostacyclin synthase expression in small and medium sized pulmonary arteries [23]. PGI2 -receptor knockout mice develop highly severe hypoxia-induced PH [24] while PGI2 receptor overexpressing mice demonstrate a protection against hypoxia induced $\mathrm{PH}[23]$.

\section{Nitric oxide (NO)}

NO is synthesized from L-arginine in endothelium by endothelial NO synthase (eNOS). It stimulates soluble guanylate cyclase (sGC) to produce cyclic guanosine monophosphate (cGMP) in PASMCs, exerting vasodilatory and anti-proliferative effects [25]. All forms of PH are believed to be associated with a state of reduced NO bioavailability, as an outcome of reduced NOS expression [26], oxidative stress [27] and/or inhibition of NO synthesis [28]. Oxidative stress leads to production of free radicals, like superoxide which react with NO to form peroxynitrite, reducing NO levels [27]. Asymmetric dimethyl arginine (ADMA), an endogenous competitor of NOS is increased in IPAH patients while enzyme responsible for its hydrolysis, dimethyl arginine dimethyl aminohydrolase (DDAH2) is decreased in IPAH patients [28].

\section{Vasoactive intestinal peptide (VIP)}

VIP, primarily functioning as a neurotransmitter, also acts as a potent pulmonary vasodilator and anti-proliferative agent [29]. Low serum concentrations and decreased VIP immuno-reactivity were shown in pulmonary arteries from IPAH patients [30].

\section{Endothelin-1 (ET-1)}

ET-1 is a potent vasoconstrictor and mitogenic factor for PASMCs and has been found to be upregulated in lungs and plasmaof patients with PH of various etiologies [31] [32]. ET-1 mediates its proliferative functions in PASMCs via binding to ET-A and ET-B receptors (ETRA, ETRB) [33]. As ETRB on endothelial cells is involved in release of vasodilators [34], hence, selective inhibition of ETRA has moved into focus in therapeutic targeting of ET-1 pathway in PH [33].

\section{Serotonin}

Serotonin (5-Hydroxytryptamine, 5-HT), produced in pulmonary artery endothelial cells (PAECs), causes vasoconstriction and remodeling in PASMCs and PAFBs in a paracrine manner, via serotonin transporter (5-HTT) and receptors [35]. Increased circulating 5-HT levels, along with increased 5-HTT expression on PASMCs are observed in PAH patients [36, 37]. 5-HTT knockout mice are protected to an extent against hypoxia-induced $\mathrm{PH}$ [38], while 5-HTT overexpressing mice demonstrate an increased severity of hypoxia induced PH [39]. 


\section{$K^{+} / \mathrm{Ca}^{2+}$ channels}

The classical function of these ion channels is to regulate membrane potential and vascular tone. Microarray studies have shown downregulation of voltage gated potassium channels (Kv channel) genes in PAH lungs [40].A selective loss of pulmonary artery smooth muscle Kv channels has been described in lung tissue from humans with PAH (notably Kv1.5) and from rats with hypoxia induced PH (Kv1.5 and Kv1.2), leading to membrane depolarization, increase in intracellular calcium and induction of vasoconstriction and proliferation [29]. Elevated expression of transient receptor potential channels (TRPC), TRPC3 and TRPC6, in PASMCs of IPAH patients, indicate important role of $\mathrm{Ca}^{2+}$ signaling through TRP channels in IPAH [41].

\subsubsection{Pro-proliferative mediators}

\section{Transforming growth factor $B$ (TGF B)}

The TGF $\beta$ superfamily comprises of a large number of growth factors that control many cellular functions including proliferation, migration, differentiation and extracellular matrix secretion and deposition. Alterations in two TGF $\beta$ family members have been linked to pathogenesis of PAH. Germline mutations in gene coding for BMPR2 have been identified in more than $60 \%$ cases of familial PAH and 10 to $30 \%$ cases of IPAH $[42,43]$. Furthermore, mutations in genes for type I TGF $\beta$ receptors ALK-1 and endoglin have been linked with severe PAH development in families with hereditary hemorrhagic telangiectasia [44].

\section{Platelet derived growth factor (PDGF)}

PDGFacts as potent mitogen and chemoattractant for SMCs [45]. An increased expression of PDGF and PDGF receptors has been demonstrated in pulmonary arteries of IPAH patients [46]. Additionally, upregulation of PDGF receptor $\beta$ (PDGFR $\beta$ ) has been observed in animal models of $\mathrm{PAH}$, contributing primarily to the proliferative and migratory phenotype of PASMCs [47]. Administration of Imatinib (PDGFR antagonist) after establishment of disease in monocrotaline and hypoxia models, dose dependently reversed the vascular remodeling and improved hemodynamics [48].

\section{Other growth factors}

Similar to PDGF, several other growth factors have been implicated in pathogenesis of PAH. Activation of tyrosine kinase receptors by these growth factors leads to initiation of intracellular signaling cascades leading to cellular proliferation, migration and apoptotic resistance.

Increased expression of vascular endothelial growth factor (VEGF) and its receptor 
(VEGFR2) has been reported in plexiform lesions found in lungs of IPAH and secondary PH patients [49].

Epidermal growth factor (EGF) was found to be co localized with Tenascin C, an ECM component, in the vascular lesions in PAH patients [50]. EGFR signaling was further demonstrated to be deregulated in MCT induced PAH rats and treatment with EGF receptor inhibitor, PKI166, induced PASMC apoptosis and increased survival of the rats [51].

Increased levels of basic fibroblast growth factor (bFGF/FGF-2) have been found in plasma and urine samples from PAH patients [52]. Recent findings demonstrate that PAECs from PAH patients produce increased levels of FGF-2, which contributes to increased proliferation of PASMCs and additionally, inhibition of FGF2 signaling using SU5402 reversed established PH in the same model [53].

\section{Survivin}

Survivin is the smallest member of family of "inhibitor of apoptosis" (IAP) proteins, known to be essentially expressed in all cancers but not in normal adult tissues [54]. It was found be exclusively expressed in remodelled pulmonary arteries in PAH patients and MCT induced PAH rats. Administration of mutant survivin with dominant negative features via inhaled adenoviral gene therapy reversed $\mathrm{MCT}$ induced $\mathrm{PAH}$, while administration of wild type survivin to normal rats induced $\mathrm{PH}[55]$.

\subsubsection{Inflammation}

Presence of inflammatory cells including monocytes, macrophages, T lymphocytes and dendritic cells in plexiform and other vascular lesions in PAH patients and occurrence of PAH secondary to classical inflammatory syndromes (HIV infection, schistosomiasis) clearly indicate a strong role of inflammation in pathogenesis of PAH [56]. In response to infection and inflammatory events, lung vascular cells produce inflammatory mediators (chemokines and cytokines), thereby recruiting the inflammatory cells.

\section{Cytokines}

Heightened levels of circulating cytokines, Tumor necrosis factor (TNF), Interlukin-1 $\beta$ and Interlukin-6 (IL-6) has been shown in IPAH patients [13]. These findings are further supported by animal models as MCT induced PH rats exhibit an increased expression of IL-6 in their lungs and rats injected with IL-6 develop PH. IL-6 knockout mice are protected to an extent against hypoxiainduced PH [57], while IL-6 overexpressing mice developed spontaneous vascular remodeling and 
$\mathrm{PH}$ [58]. Further data suggested interaction between IL-6 and BMPRII where loss of BMPRII in mice lead to upregulation of IL-6 production [59] and increased IL-6 levels were found to suppress expression of BMPRII [60].

\section{Chemokines}

Chemokines play a role in leukocyte recruitment and trafficking in $\mathrm{PH}$, such as rolling, activation, adhesion and extravasation into the inflamed tissue along a chemoattractant gradient [56]. Fractalkine (CX3CL1) a soluble chemotactic protein which is also found as a membrane cell adhesion molecule on ECS, promotes chemokine receptor 1 (CX3CR1) expressing leukocyte recruitment [13]. CXCL3 expression is found to be upregulated in circulating CD4+ and CD8+ Tlymphocytes and inflammatory cells surrounding PA lesions in IPAH patients [61]. Other chemokines upregulated in PAH patients are RANTES (CCL5) and chemokine ligand 2 (CCL2). Elevated levels of CCL2 are produced from PAECs of IPAH patients and interestingly, PASMCs isolated from the patients also exhibit a stronger migratory and proliferative response to CCL2 as compared with control subjects [62].

\subsubsection{Treatment approaches for PAH}

Pulmonary Hypertension is characterized by poor prognosis and until now, no cure exists for the disease. However, improved understanding of the patho-biological mechanisms of the disease has resulted in the development of effective therapies targeting specific aberrant pathways. At present, there are three classes of vasodilatory medications available : prostanoids, endothelin receptor antagonists and phosphodiesterase-5 (PDE-5) inhibitors, which have shown efficacy in treatment of PAH by targeting different pathways involved in proliferation and contraction of PASMCs (Figure 1.3, green lines). Several other potential pathways targeting pulmonary vascular remodeling have been identified and are under investigation for drug development (Figure 1.3, red lines). 


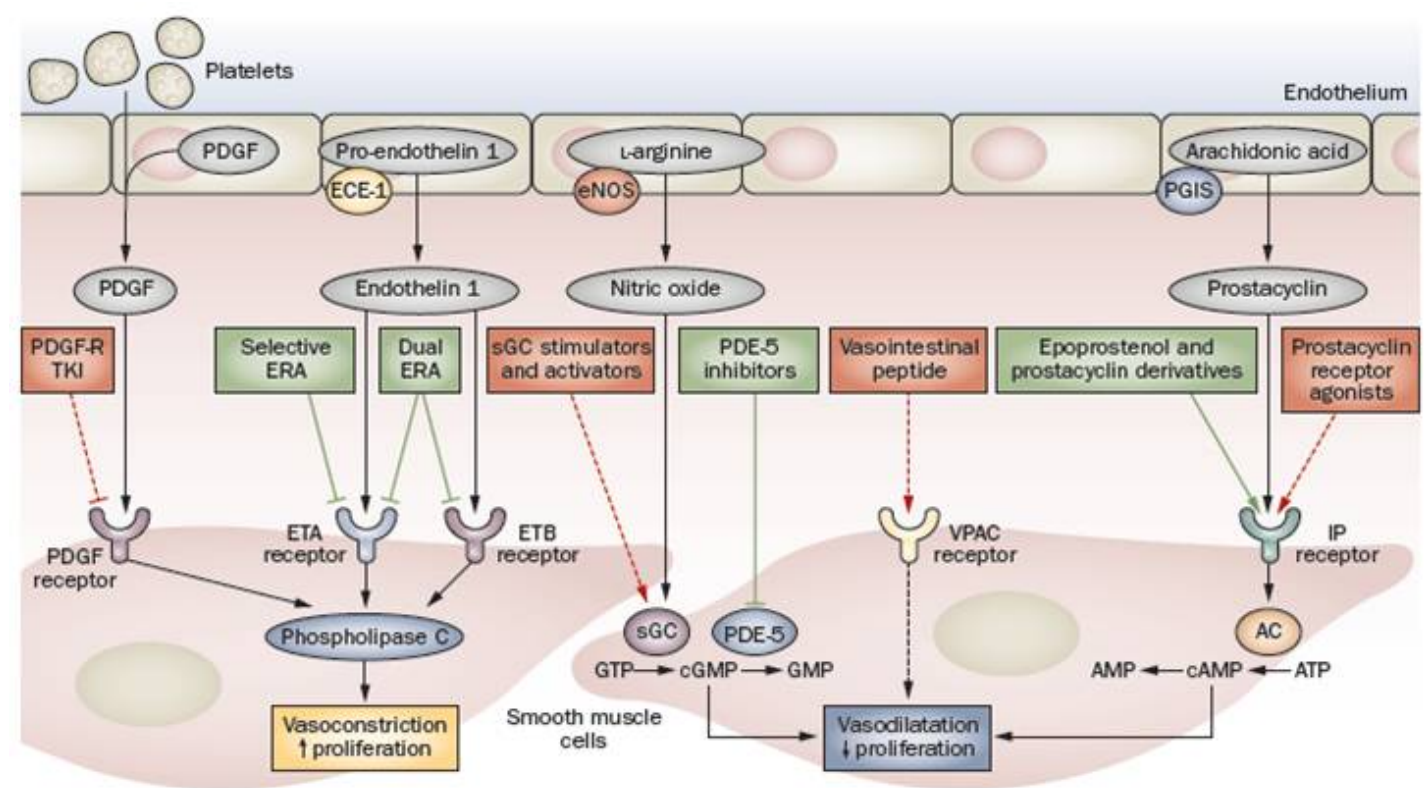

Figure 1.2 Current and emerging targets and therapies for Pulmonary Arterial Hypertension. Pulmonary artery smooth muscle cell therapeutic targets and corresponding licensed (green boxes) and investigational (red boxes) treatment approaches for pulmonary arterial hypertension. Arrows represent receptor stimulation, whereas terminated lines show receptor blockade. AC, adenylate cyclase; cAMP, cyclic AMP; cGMP, cyclic GMP; ECE-1, endothelin converting enzyme 1; eNOS, endothelial nitric oxide synthase ; ETA, endothelin receptor type $A$; ETB, endothelin receptor type $B$; ERA, endothelin receptor antagonists; IP, prostaglandin 12; PDE-5, phosphodiesterase type 5; PDGF, platelet derived growth factor; PDGF-R TKI, PDGF receptor tyrosine kinase inhibitors; PGIS, prostaglandin I synthase; SGC, soluble guanylate cyclase; VPAC, vasointestinal peptide receptor. (O'Callaghan et. al., Nat. Rev. Cardiol., 2011) [63]

\subsubsection{Currently available therapies}

\section{Prostanoids}

Endothelium derived prostacyclin is a potent pulmonary vasodilator, also exerting antiproliferative and anti-thrombotic effects [22]. Intravenous Epoprostenol was the first approved therapeutic approach to show improved conditions and survival in PAH patient [64]. Due to the disadvantage of short half-life and systemic side effects, new prostacyclin analogs with longer halflife and alternative routes of administration were developed. They include: Treprostenol with halflife of 4.5 hours [65], Iloprost [66] and Beraprost [67], administered intravenous, subcutaneous or by inhalation in $\mathrm{PAH}$ patients.

\section{Endothelin receptor antagonists (ERAs)}

ET-1, produced by endothelial cells, is a potent vasoconstrictor and PASMCs mitogen playing an important role in pathogenesis of PAH [31]. Bosentan, an oral non selective antagonist (binding to both receptor A and B), has proved its efficacy for treatment of PAH in two randomized clinical trials [68].However, severe liver dysfunction has been reported in a significant number of 
patients taking the drug. As binding of endothelin to receptor $A$ has been described in vascular remodeling, ERA specific antagonists, Sitaxentan [69] and Ambrisentan [70] were developed. Though Sitaxentan was withdrawn from the market in 2010 as an outcome of a number of patients death due to acute liver failure [63], Ambrisentan is an approved drug for PAH. A novel, highly potent, tissue-targeting ERA, Macitentan characterized by high lipophilicity is under investigation [71].

\section{Phosphodiesterase-5 (PDE-5) inhibitors}

PDE-5, enzyme abundantly found in pulmonary vasculature, inactivates cGMP, the secondary messenger responsible for mediating the vasodilatory activity of NO [25]. Hence, PDE-5 inhibitor, Sildenafil, initially used for erectile dysfunction, has emerged as an important drug therapy for PAH [72, 73]. Beneficial effects including improved symptoms, hemodynamics, exercise capacity and survival have been shown by a one-year extension study after a daily oral treatment with sildenafil for 12 weeks [73]. Another PDE-5inhibitor, Tadalafil, having the advantage of once-daily dosing, has been granted regulatory approval for use in patients with PAH in 2009 [74].

\subsubsection{Emerging treatment approaches}

VIP

As described above (section 1.2.3.1), VIP has been documented to contribute to the pathogenesis of PAH, suggesting that targeting VIP might be a useful approach. Aviptadil, VIP analog, administered by inhalation, has demonstrated modest increase in cardiac output along with transient vasodilation and increased stroke volume in PAH patients with various etiologies [75].

\section{sGC stimulators and activators}

As the therapeutic effect of PDE-5 inhibitors is dependent on baseline NO expression, typically reduced in PAH [76], therefore, treatments that act directly on soluble Guanalyte cyclase (sGC) could potentially have a greater efficacy than them. Interestingly, one such NO independent SGC stimulator, Riociguat has proven to be effective in hypoxia induced $\mathrm{PH}$ in mice and MCT induced PAH in rats [77]. Improvements in symptoms, NYHA functional class, exercise capacity, NT-proBNP level, and pulmonary hemodynamics was observed in PAH and CTEPH patients in Phase II trials with oral Riocioguat for 12 weeks [78]. Preliminary results of Phase III trials are expected soon. 


\section{Prostacyclin receptor agonists}

Effectiveness demonstrated by prostanoids in therapy of PAH has led to development of a new class of non-prostanid agonists specific for prostacyclin IP receptors. Selexipag is first-in-class orally active prodrug which is metabolized to highly selective IP receptor agonist MRE-269, having half-life of over 6hrs [79]. Treatment of 43 patients with this drug in Phase Ila trials has shown significant improvements in PVR values and 6 min walk distance, leading to phase III randomized trials.

\section{Tyrosine kinase inhibitors}

The current therapeutic approaches as well as above mentioned emerging strategies mainly provide symptomatic relief and some improvement in prognosis. Thus, a need of compounds that reverse the remodeling observed in the disease has led to use of receptor tyrosine kinase inhibitors (RTKs) in PAH. Most promising drug from this class is Imatinib (ST1571), an orally active selective inhibitor of RTK BCR-ABL and PDGFRß, currently licensed for treatment of chronic myleogenous leukemia (CML) and gastrointestinal tumors. RTKs including PDGFR $\beta$ have been implicated in pathogenesis of PAH in humans and in rodent $\mathrm{PH}$ models (as discussed in section 1.2.3). Imatinib was shown to reverse experimental pulmonary hypertension via inhibition of PDGFR $\beta$ signaling [48].

The drug was first used in patients with advance disease receiving other PAH specific drugs [80] and in PAH patients with CML [81] in 2005. Results showed favourable clinical and hemodynamic responses. Enrolment for the phase III randomized clinical trials has been completed and preliminary results are expected soon [63].

The muitikinase inhibitor, Sorafenib and the EGFR inhibitor, Iressa, have already shown to attenuate vascular remodeling in the animal models of $\mathrm{PH}[51,82]$ and appear promising for future therapy of PAH.

\subsection{Notch Signaling Pathway}

Notch gene was discovered more than 90 years ago and was named after the notched wing phenotype observed in mutant Drosophila [83]. It is an evolutionarily conserved signaling pathway essential for cell-fate determination during embryonic development in organisms ranging from sea urchins to humans [84]. There are many unique features of this cascade that sets it apart from other signaling pathways. Firstly, both the receptors and ligands are transmembrane proteins. Secondly, signaling relies on proteolytic cleavage of the receptor due to ligand binding, releasing 
an active fragment which translocates to the nucleus and activates transcription of downstream target genes [85].

\subsubsection{Components of Canonical Notch Signaling Pathway}

\section{Notch receptors}

Notch receptors are large single pass type I transmembrane proteins first identified in Drosophila [86-88] and later in mammals, Notch1 [89], 2 [90], 3 [91] and 4 [92]. Drosophila melanogaster possesses only one Notch receptor, C. Elegans possesses two redundant notch receptors while mammals have 4 notch receptors (Notch1-4), displaying both redundant and unique functions [85]. Notch is synthesized as a single polypeptide in ER where it is further fucosylated by O-fucosyl- transferase (OFUT1) and glycosylated by O-glucosyl-transferase (Rumi). It is then translocated to Golgi where it is cleaved by Furin protease at the S1 site and further, modified by $\mathrm{N}$-acetylglucosaminyltransferase, Fringe [93].

Notch receptor is displayed on cell surface as a heterodimer, consisting of an $\mathrm{N}$-terminal extracellular domain joined non-covalently to C-terminal fragment consisting of transmembrane domain and intracellular domain. The $\mathrm{N}$ terminal part of notch extracellular domain consists of EGF (Epidermal growth factor)-like repeats and LNRs (lin-12 Notch repeats). In mammals, EGF repeats 11 and 12 interact with the ligands. NICD (Notch Intra-cellular Domain) consists of N terminal RAM (recombination binding protein-Jk associated molecule) domain, an ankyrin domain, and less conserved regions, a variable trans activation domain and a C-terminal PEST (Pro-Glu-SerThr) degradation sequence (Figure 1.4) [94]. In addition, Notch receptors 1-3 contain two nuclear localization signals (NLS) compared to one NLS in Notch4.

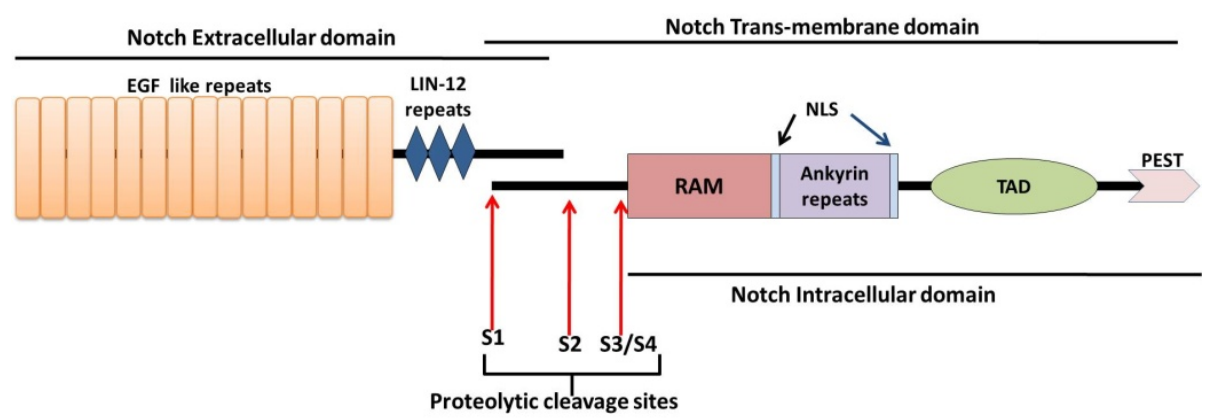

Figure 1.3 Schematic representation of general domain organization of Notch pathway receptors in mammals. NLS, nulclear localization signal; TAD, transcriptional activation domain PEST, Pro-Glu-Ser-Thr; RAM, RBP-J association molecule domain. 


\section{DSL Ligands}

The canonical DSL (Delta, Serrate, Lag2) ligands are responsible for most of Notch signaling mediated effects. Mammals have 5 DSL family ligands. On the basis of structural homology to the Drosophila ligands, the mammalian ligands are either termed as Delta Like (DLL1, DLL3 and DLL4) or Serrate like (Jagged1 and Jagged2). Like the Notch receptors, DSL ligands are also transmembrane proteins having extracellular domain consisting of tandem EGF repeats and a cysteine rich N-terminal DSL domain [95]. DSL domain mediates binding of the ligands to the Notch receptors [96]. The intracellular regions lack sequence homology except some, contain multiple lysine residues and a C-terminal PDZ (PSD-95/Dlg/ZO-1) ligand motif, needed for ligand signaling activity and interactions with the cytoskeleton respectively (Figure 1.5) [97].

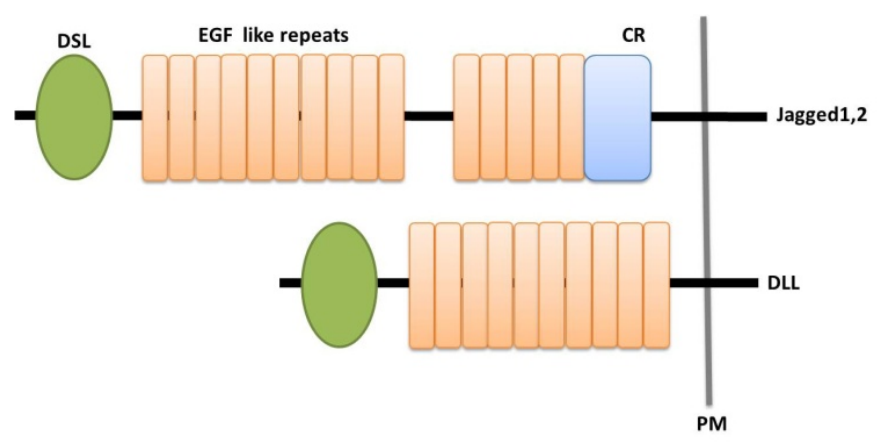

Figure 1.4 Schematic representation of domain organization of DSL ligands in mammals. DLL, Delta like ligand; DSL, Delta Serrate Lag2; EGF, epidermal growth factor; PM, plasma membrane; CR, cysteine rich domain.

\section{$\boldsymbol{v}$-Secretase complex}

Initial clues for identification of the $\gamma$-Secretase complex came from genetic studies of autosomal dominant forms of familial Alzheimer's disease (AD) in mid 1990s. Missense mutations in two unknown genes, Presenilin 1 and 2 (Psen-1 and 2) were sufficient to cause an aggressive and inherited form of $A D$, though their proteolytic functions were identified much later $[98,99]$. Psen-1 knockout not only revealed a notch phenotype but also, substantiated its requirement for $\gamma$-secretase proteolytic activity [100]. Since then, $\gamma$-secretase complex has been shown to have three additional proteins, Nicastrin, Aph and Pen-2 [98]. Psen1 forms the catalytic core of the complex, though the other three proteins are also needed for proper $\gamma$-secretase activity [101]. Two conserved aspartyl residues, located within transmembrane domain 6 and 7 harbor the catalytic activity [102]. 


\section{CSL transcription factor}

Key effector molecule of the Notch signaling pathway is a DNA binding protein, CSL ((CBF1/Suppressor of Hairless/LAG-1), which is similar to the Rel family of transcription factors. Like the Rel family, CSL has the two Rel-homology domains (RHR-N, RHR-D), required for DNA binding and dimerisation, along with an extra $\beta$-trefoil domain (BTD) between the two Rel domains. This BTD domain is believed to mediate interaction with NICD [94]. The target DNA sequence for CSL is CGTGGGA [103]. Interaction between Notch intra-cellular domain and CSL transcription factor became clear when a nuclear complex bound to a promoter regulated by Notch was shown to contain CSL and Notch [104].

\section{Notch target genes}

Although signals mediated through the Notch receptors have diverse outcomes, only a limited number of Notch target genes have been identified. These genes belong to the family of bHLH (basic helix loop helix) transcription factors, namely, HES (hairy/enhancer of split) and HERP (Hes related-repressor protein, also called HEY) family [83]. Both families share structure similarities like bHLH domain, responsible for DNA binding and hetero- or homo-dimerization and orange domain. The most striking difference between Hes and Hey proteins is replacement of WRPW tetrapeptide in former to YRPW peptide in latter (Figure 1.6). In mammals, seven Hes (Hes1-7) and three Hey (Hey1, 2 and L) genes have been identified, which are highly similar in structure to their Drosophila ancestors, Hairy, E (spl) and Hey [105]. Out of these, all three Hey genes and Hes1, 5 and 7 are known to be induced by Notch, as activation of their promoters by NotchICD over expression was observed in reporter gene assays [106].

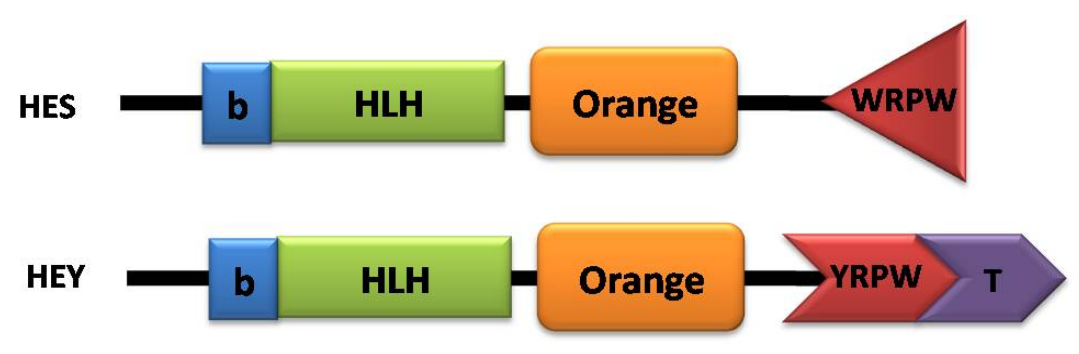

Figure 1.5 Domain organization of Hes and Hey proteins. HLH, helix-loop-helix; YRPW, tyrosine-arginineprolie-tryptophan; WRPW, tryptophan-arginine-proline-tryptophan.

Though Hes and Hey genes are the major targets of notch pathway, other targets have also been identified, as their promoters harbour CSL binding sites. Some of the notch target genes 
identified are c-myc [107], cyclin D1 [108] and p21/Waf1 [109]. Most importantly, regulation of these genes is tissue-specific and context-dependent.

\subsubsection{Notch signaling cascade}

Notch signaling is triggered on direct cell-cell contact. Interaction between Notch receptors and ligands (Jagged or DLL), expressed on adjacent cell membranes results in activation of signaling. Binding of the ligand, triggers two proteolytic cleavages in Notch receptor. A Disintegrin And Metalloproteinase (ADAM)-family of proteases catalyses first cleavage called S2 cleavage generating membrane -anchored Notch extracellular truncation (NEXT) fragment. The NEXT fragment acts as a substrate for gamma-secretase enzyme complex. $\gamma$-secretase mediated S3/S4 cleavage releases Notch intra-cellular domain, which then translocates to the nucleus. In nucleus, NICD binds to the DNA binding protein CSL, also called RBJK (recombination signal sequencebinding protein JK). In absence of NICD, CSL is associated with ubiquitous corepressor proteins or histone deacetylases (HDACs) to prevent transcription of target genes. Upon NICD binding, allosteric changes occur in CSL, facilitating displacement of repressors. Coactivators, including MAML (Mastermind-like), CBP and others are subsequently recruited, leading to transcription of Notch target genes (Figure 1.7) [83, 110, 111].

MAML further recruits CyclinC/ Cdk8 pair which strongly enhances NICD phosphorylation. This facilitates unbiquitinylation of NICD by the Fbw7/ Sel10 ubiquitin ligase resulting in rapid NICD degradation and formation of RBP-JK corepressor complexes [112].

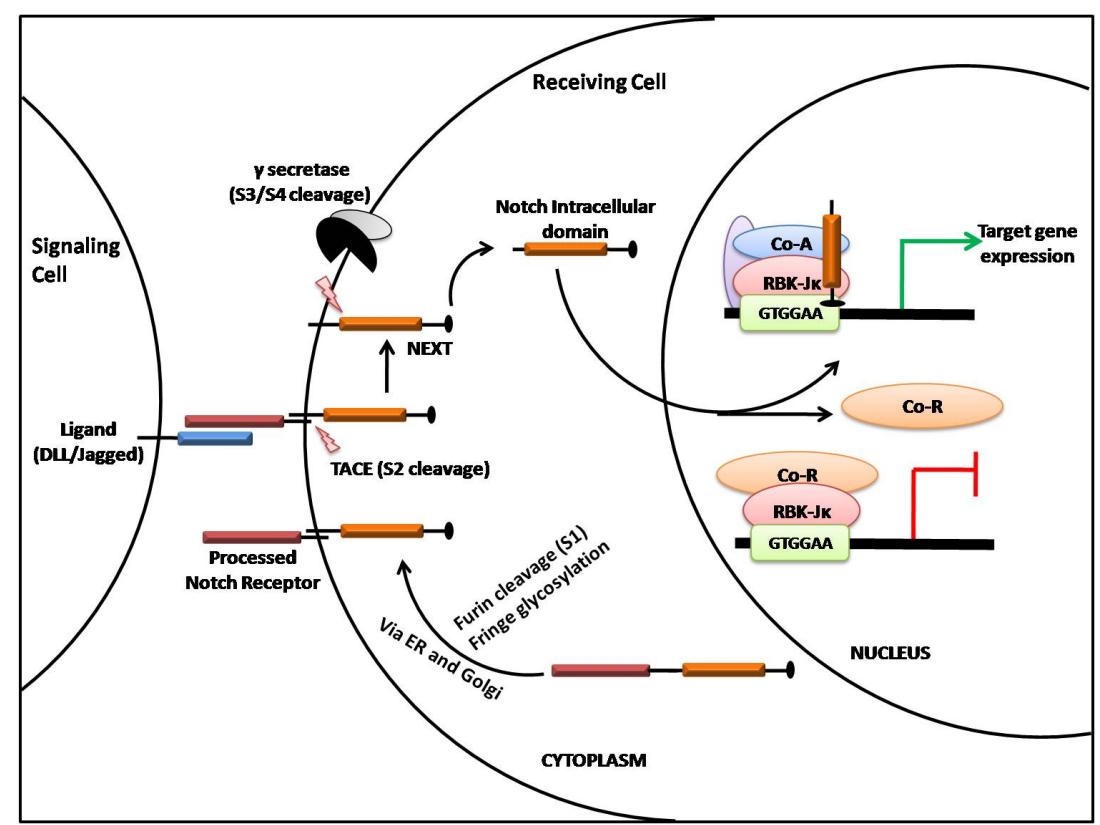

Figure 1.6 Schematic representation of canonical Notch signaling Pathway. Co-A, coactivators; Co- 
R,corepressors; ER, endoplasmic reticulum; NEXT, notch extracellular truncation fragment; RBK-JK, recombination signal sequence binding protein JK; TACE, tumor necrosis factor- $\alpha$ converting enzyme.

\subsubsection{Functions of Notch signaling pathway}

Notch Signaling controls numerous cellular processes like stem cell maintenance, cell fate specification, differentiation, proliferation and apoptosis. The three main ways of its function are:

\section{Stem cell maintenance}

Signal from the ligand expressing differentiated progeny cells prevent the notch expressing precursor cells to differentiate and thus, maintain stem cell characters (Figure 1.8a) as observed in vertebrate nervous system where Notch signaling is believed to maintain balance between precursor and differentiated cell pools $[113,114]$.

\section{Binary cell fate decision}

This term refers to a condition where precursor cell has to choose between two different cell fates. Notch signaling influences binary cell fate decisions by:

Lateral signaling: Initially two equipotent cells express equal amounts of the ligand (L) and notch $(\mathrm{N})$ but gradually the concentrations start differing. The mechanism behind this change remains unknown. The ligand expressing cells send activating signals to notch expressing cells adopting different cell fates (Figure 1.8b) [115].

Inductive signaling: It involves signaling between two different cell types where a precursor cell is instructed to differentiate into a particular cell fate after interacting with a distinct neighboring cell expressing ligands. In absence of the signal from ligands, precursor would adopt another fate (Figure 1.8c) [116].

\section{Induction of terminal differentiation}

Notch signaling between two developmentally related cells could initiate terminal differentiation and induce the cells to exit from cell cycle (Figure 1.7d). For example, DLL1 induced notch signaling was shown to initiate terminal differentiation program in keratinocytes from human skin [117]. 

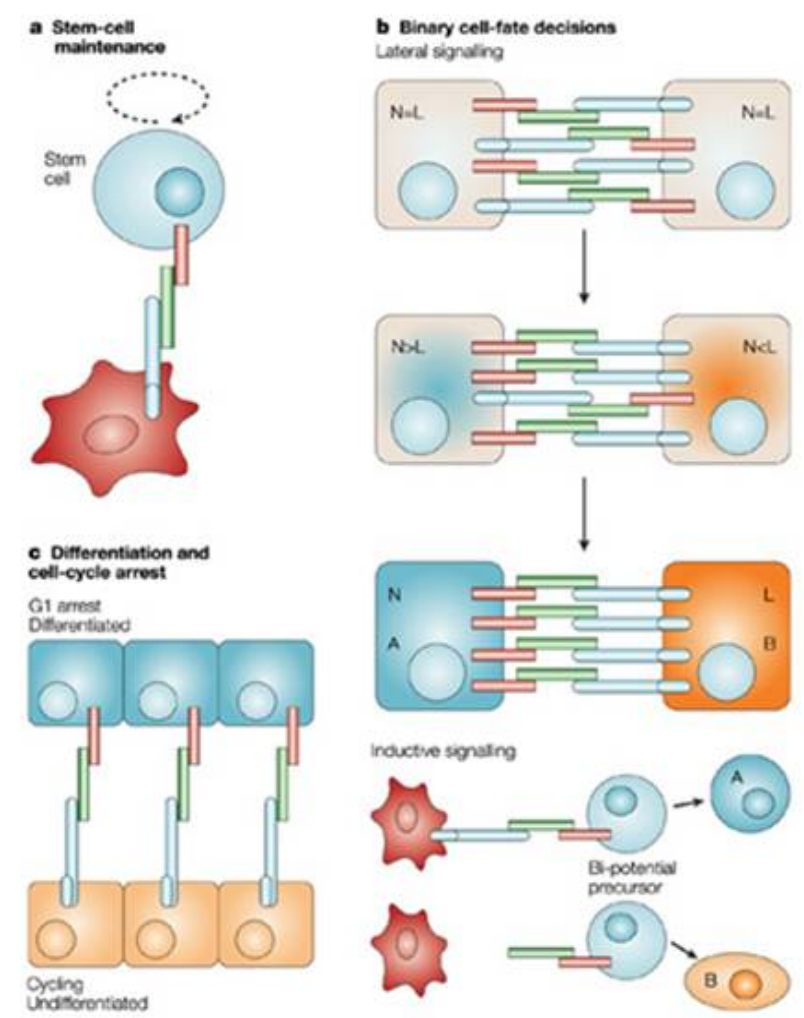

Figure 1.7 Pleiotropic effects of Notch signaling. Three main effects are represented schematically. (a) Notch signaling can maintain stem cells or precursor populations in an undifferentiated state. (b) Notch signaling influences binary cell-fate decisions via lateral or inductive signaling. (c) A third property of Notch is its ability to influence differentiation and cell-cycle progression. (Radtke and Raj, Nat. Rev. Cancer, 2003) [114]

\subsubsection{Notch signaling in vasculature}

\subsubsection{Notch and vascular development}

Transgenic studies in mice, involving loss and gain of function, have shown that Notch signaling is essential for vascular development. Altering notch signaling leads to abnormal vascular development at different stages and to various degrees. Notch1 homozygous knockout results in embryonic lethality due to defects in somitogenesis and cardiovascular abnormalities [118]. Cre mediated deletion of endothelial Notch1 during embryonic development is also lethal, proving that arrested growth is associated with loss of gene in vessels/endocardium [119]. Double knock out of Notch4 and Notch1, have a more severe vascular phenotype than embryonic Notch1 knock out [118], indicating that though Notch4 is not essential for embryonic vascular development, Notch1 and Notch4 have partially over lapping functions. Moreover, endothelial specific gain of function of Notch 4 also reveals vascular defects [120], indicating that precise levels of Notch are necessary for vascular development. Vascular defects and lack of arterial specific markers in CSL 
knock outs provide additional argument supporting importance of notch signaling in vascular development [121].

Analysis of zebrafish embryos lacking Notch signaling gave initial clue that primary function of Notch signaling in vascular development is specification of arterial fate in endothelial cells. These embryos exhibit decreased expression of arterial markers like ephrinB2 with expansion of venous markers [122]. Notch was found to act downstream of VEGF and sonic hedgehog (shh) pathway via activation of gridlock (zebrafishortholog of mammalian Hey) to regulate arterial identity [123]. Furthermore, Notch3 null mice exhibit marked arterial defects including enlarged arteries with abnormal distribution of elastic laminae, revealing Notch3 as a gene necessary for differentiation and acquisition of arterial identity of vascular smooth muscle cells [124, 125].

In addition to these findings, the role of notch signaling in vascular development is highlighted by the fact that mutations in notch signaling components leads to inherited vascular disorders. Mutations in Notch3 gene causes cerebral autosomal dominant arteriopathy with subcortical infarcts and leukoencephalopathy (CADASIL) characterized by adult onset conditions of stroke and dementia [126]. The disease is associated with progressive degeneration of vascular smooth muscle cells (vSMCs) and accumulation of granular osmiophilic material (GOM) in smooth muscle cell vascular membrane [127].

\subsubsection{Notch signaling in adult vasculature}

All four notch receptors are expressed in adult vasculature. Notch1, Notch2 and Notch4 have been reported to be expressed in endothelial cells in vivo, while Notch3 expression appears to be restricted to the smooth muscle cells. Among ligands, only Jagged1, Jagged 2 and DLL4 are found to be involved in activating Notch signaling in vasculature [128]. Notch4 activation was shown to promote survival in endothelial cells by up regulating anti-apoptotic protein Bcl-2 [129]. On the other hand, constitutive activation of Notch ICD or activation of Notch pathway by Jagged1 inhibited proliferation of endothelial cells by upregulating p21Cip1 [130,131]. Expression of Notch3 is shown to repress expression of genes encoding contractile proteins and to promote proliferation in vSMCs $[132,133]$. During hypoxia, hypoxia inducible factor $1 \alpha$ (HIF-1 $\alpha$ ), is shown to interact with NICD to activate notch responsive genes, to maintain cells in undifferentiated state [134]. Notch3 is also shown to regulate expression of platelet-derived growth factor receptor $\beta$ in vSMCs [135].

Several studies have characterized expression of notch pathway genes in response to 
vascular injury [132, 136-138]. The expression of several Notch pathway components, comprising of Notch1, Notch3, Jag1, Jag2 and downstream target genes, Hey1 and Hey2 are regulated in a temporal manner after experimentally induced vascular injury. Decreased neointima formation in heterozygous $\mathrm{Notch}^{+/-}$mice [139] and $\mathrm{Hey}^{-/-}$[140] mice after carotid artery ligation, provide strong evidence for modulation of notch signaling components during response to vascular injury.

\subsubsection{Targeting the Notch signaling pathway}

Accumulating evidence support the role of Notch signaling in several diseases, in particular cancer [141], has led to an increased interest in therapeutic modulation of the pathway. Many genetic and pharmacologic approaches are either available or theoretically possible to block the signaling at different levels (Figure 1.8). Targeting could be achieved by selective strategies including monoclonal antibodies and RNA interference; non-selective strategies comprising of soluble ligands and receptor decoys; or inhibition of enzymes involved in glycosylation or cleavage of receptors, like $\gamma$-secretase inhibitors (GSIs) and ADAM inhibitors [142, 143].

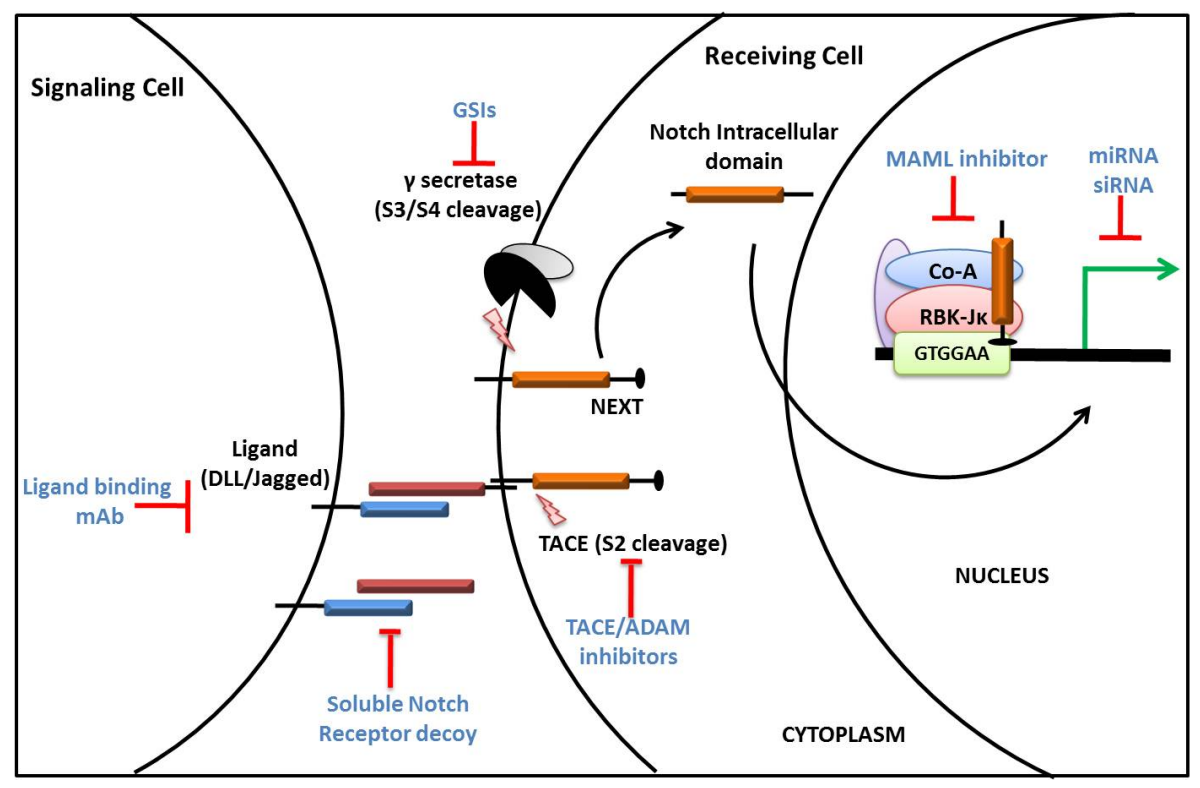

Figure 1.8 Schematic representation of putative therapeutic targets in Notch Signaling. (Red) Flat tiped arrows indicate inhibition. GSIs, $\gamma$ secretase inhibitors; $\mathrm{mAb}$, monoclonal antibody; MAML, mastermind like; miRNA, micro RNA.

GSIs, a class of small molecule inhibitors of $\gamma$-secretase enzyme, form the pioneering group of notch inhibitors, both in lab and clinic. They were found to be active in several experimental models of tumors and some of them, MK-0752 (Merck) and R04929097 (Roche) have already entered clinical trials for breast cancer [143]. GSIs have the advantage of relative ease of 
administration, oral bioavailability and low cost. Furthermore, a single molecule can block the cleavage of all four Notch homologs which may be favorable in conditions where more than one Notch receptors needs to be targeted. However, main challenges with use of GSIs are their offtarget effects as $\gamma$-secretase has numerous targets other than notch and gastrointestinal toxicity observed in pre-clinical models [144] and early clinical studies. Though, intermittent administration of GSIs was shown to greatly ameliorate the intestinal toxicity [142].

A more specific alternative to GSI based therapy is to use antibodies directed against receptors, ligands or other components of the pathway. In vitro studies on human tumor lines indicated that antibodies targeting Notch1 and Notch3 were able to inhibit oncogenic Notch signaling, though not as potently as cell-penetrating, small molecule GSI compounds [145]. Several Notch-targeting antibodies are now being evaluated in preclinical studies, and the anti-Notch monoclonal antibody OMP-59R5 is in phase I clinical trials [146].

\subsubsection{Notch signaling in Pulmonary Arterial Hypertension}

Notch3 and its downstream target gene, Hes5 were found to be elevated in lung biopsies as well as in PASMCs of non-familial PH patients. In vitro, Notch3 induced a proliferative phenotype in PASMCs which was obliterated by Hes 5 knock down. Interestingly, Notch3 ${ }^{-/-}$mice were resistant to development of hypoxia induced PH. Furthermore, treatment with DAPT, a $\gamma$ secretase inhibitor, effectively attenuated hypoxia induced PH in mice [147]. This work elegantly addresses the role of Notch3 in phenotypic alterations of PAH-PASMCs. However, the expression and regulation of different Notch receptors and ligands in human PAH as well as in experimental models of PH need to be addressed in detail. Most importantly, the role of Notch signaling in initiation/ progression of complex vascular lesions in PH remains unexplored.

\subsection{Animal models of Pulmonary Hypertension}

Recent advances in our understanding of the pathophysiological mechanisms underlying the pathogenesis of $\mathrm{PH}$ can be attributed to the use of experimental animal models. These animal models have not only been the source of scientific information such as role of molecular mechanisms and genetic contributions in $\mathrm{PH}$ but also, played an indispensable role in discovery and testing of possible therapeutic approaches.

\subsubsection{Monocrotaline (MCT) induced PAH model}

Initially used over 40 years ago, it is the oldest classical model used in field of PAH. MCT, a 
toxic pyrrolizidine alkaloid obtained from plant Crotalaria Spectabilis, is injected once either intraperitoneally or subcutaneously to induce PAH. Injected MCT is metabolized in liver by cytotchrome P450 3A to MCT pyrole, the active form. Severe pulmonary vascular disease occurs within a few days, followed by pulmonary vascular remodeling (Figure 1.3) and elevated PAP [148]. Basic underlying mechanism of MCT induced PAH is unknown, though direct endothelial damage triggering development and progression of severe $\mathrm{PH}$ [149] and infiltration of mononuclear inflammatory cells into adventitia leading to medial hypertrophy [150] have been suggested. Response to MCT varies among strains and species of animals. While rats are the preferred choices, exhibiting consistent and predictable response, and mice are resistant to the effects of MCT. Despite being used extensively, this model is not perfect for human PAH as neointima lesions observed in PAH patients are not formed in MCT rats. Moreover, it is relatively easy to cure as so far 30 agents have been reported to prevent or cure the remodeling, very unlike the human with PAH [151].

\subsubsection{Chronic Hypoxia (CH) model of PH}

Normo- and hypo-baric hypoxia are commonly used to induce PH in wide variety of animal species. Chronic hypoxia in rats exhibit pulmonary vasoconstriction, medial hypertrophy, and increased muscularization of the small arteries with elevated smooth muscle actin (Figure 1.3) [148], mimicking human disease pathogenesis. Though $\mathrm{CH}$ rats develop more severe vascular remodeling as compared to mice, but availability of genetically modified strains of mice has led to more frequent use of $\mathrm{CH}$ model in mice. Exposure of mice to chronic hypoxia is associated with minimal vascular remodeling causing an elevation in PAP and RV hypertrophy. The most common pathological findings are muscularization of previously non-muscularized walls and a moderate medial thickening of muscular resistance walls [151]. $\mathrm{CH}$ mouse model has been used to investigate the role of NO pathway, reactive oxygen species (ROS) and cytoskeletal architecture in development of $\mathrm{PH}[77,152,153]$.

The main limitation of this model is that $\mathrm{CH}$ induced $\mathrm{PH}$ in mice and rats is reversed once the animals are exposed to normal oxygen concentrations [148]. 


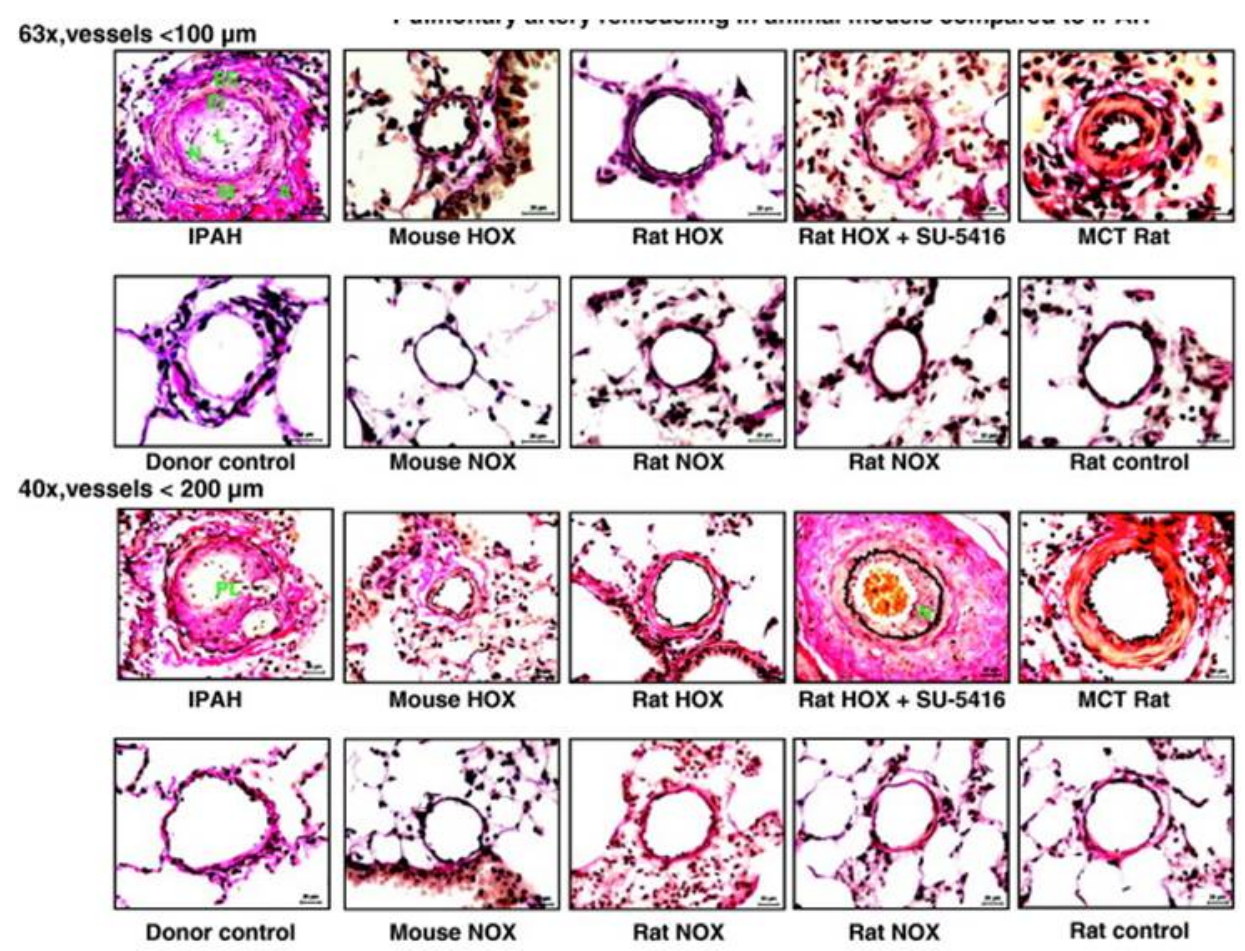

Figure 1.9 Pulmonary artery remodeling in animal models compared to IPAH. Staining, elastic van Gieson. Elastic fibers, blue/black/brown; nuclei, black/brown; collagen fibers, red; media, epithelia, nerves, erythrocytes, yellow; IPAH, idiopathic pulmonary arterial hypertension; HOX, 3 weeks hypoxic exposure; NOX, normoxic control; MCT, monocrotaline injection; EE, elastic externa; El, elastic interna; L, lumen; $N$, neointima, M, media; A adventitia; PL, plexiform lesion. (Pak et.al., Drug Discovery Today, 2010) [154]

\subsubsection{Chronic Hypoxia with Su5416 rat model}

In contrast to the human disease, both above mentioned classical models, MCT and Chronic hypoxia model, lack formation of plexiform lesions, clustered proliferative endothelial cells in lumen of pulmonary arteries. A modification of the $\mathrm{CH}$ model involving administration of SU5416, vascular endothelial growth factor receptor 2 (VEGFR2) antagonist, to $\mathrm{CH}$ rats has been developed, which emulates the hyperproliferative endothelial cell etiology and irreversible $\mathrm{PH}$ (Figure 1.3) [155]. It was postulated that VEGFR2 blockade with SU5416 initiates an initial endothelial cell apoptosis and this endothelial cell death together with chronic hypoxia results in selection of apoptosis resistant hyper-proliferative endothelial cell phenotype. Because of the severity of $\mathrm{PH}$, the formation of neointimal lesions and the progressive nature of the model, this model has been used to evaluate new treatments for PAH such as bradykinin antagonist, B-9430 [156] and the anticancer drug, Sorafenib [155]. 


\section{AIM OF THE STUDY}

Despite the plethora of scientific work carried out in the field of pulmonary hypertension, clinical interventions can only alleviate the symptoms but cannot cure the disease.

In the cardiovascular system, Notch signaling modulates multiple aspects of vascular development including vasculogenesis, angiogenesis and differentiation of vascular smooth muscle cells. Notch signaling molecules are not only instrumental in vascular development, but also key mediators in pathological conditions, including vascular injury. Hence, we hypothesized that Notch signaling pathway may be involved in pathogenesis of $\mathrm{PH}$.

The overall focus of the work presented in this thesis is on elucidating the role of Notch Signaling in PH. Specifically, we aimed to:

- Study the expression profile of Notch receptors and ligands in different animal models of $\mathrm{PH}$.

- Study the expression profile of Notch receptors and ligands in lungs and pulmonary arteries from patients with IPAH and donors.

- Investigate the localization of candidate Notch in lungs from patients with IPAH and Hox+Su5416 rats.

- Investigate the effect of candidate Notch receptor knockdown and overexpression on proliferation of hPAECs and hPASMCs.

- Investigate the effect of candidate Notch receptor knockdown and constitutive activation on survival of hPAECs.

- Investigate the effect of pan Notch inhibitor ( $\gamma$ secretase inhibitor) on proliferation of hPAECS and hPASMCs in vitro. 


\section{MATERIALS AND METHODS}

\subsection{Materials}

\subsubsection{Reagents and Chemicals}

\begin{tabular}{|c|c|}
\hline Reagent & Company \\
\hline Acetic acid, glacial 99\% & Sigma, USA \\
\hline Acrylamide solution (30) & Sigma, USA \\
\hline Agarose & Carl Roth, Germany \\
\hline Ammonium Persulfate & Sigma, USA \\
\hline Ampicilin & Sigma, USA \\
\hline$\beta$ mercaptoethanol & Sigma, USA \\
\hline Bromophenol Blue & Roche, Germany \\
\hline Bovine serum albumin & Carl Roth, Germany \\
\hline BSA solution $(2 \mathrm{mg} / \mathrm{ml})$ & BioRad, USA \\
\hline Chloroform & Carl Roth, Germany \\
\hline DAPI & Invitrogen, USA \\
\hline Dimethyl sulfoxide & Sigma, USA \\
\hline Digest All 2 (Trypsin) & Invitrogen; USA \\
\hline DNA ladder (100bp,1Kb) & Fermentas, USA \\
\hline Ethanol absolute & Carl Roth, Germany \\
\hline Ethidium Bromide & Carl Roth, Germany \\
\hline Ethylenediamine- Tetraceticacid (EDTA) & Carl Roth, Germany \\
\hline Fluorescence mounting medium & Dako, USA \\
\hline Formaldehyde & Carl Roth, Germany \\
\hline Glycerol & Sigma, USA \\
\hline Glycine & Sigma, USA \\
\hline Hydrochloric acid & Carl Roth, Germany \\
\hline IQ SYBR ${ }^{\circledR}$ Green Supermix & BioRad, USA \\
\hline Isopropanol & Carl Roth, Germany \\
\hline JM109 competent cells & Promega, USA \\
\hline Luria Broth agar & Sigma, USA \\
\hline Luria Broth & Sigma, USA \\
\hline Lipofectamine 2000 & Invitrogen, USA \\
\hline Methanol & Carl Roth, Germany \\
\hline Milk powder & Carl Roth, Germany \\
\hline $\mathrm{N}, \mathrm{N}, \mathrm{N}^{\prime}, \mathrm{N}^{\prime}$-Tetramethyl-1,2-diaminomethane (TEMED) & Sigma, USA \\
\hline Ponceau S Solution & Sigma, USA \\
\hline Potassium dihydrogenphosphate & Carl Roth, Germany \\
\hline Potassium phosphatemonobasic & Carl Roth, Germany \\
\hline Rainbow protein molecular weight marker & AmershamBiosciences, USA \\
\hline RIPA lysis Buffer & Thermo Scientific, USA \\
\hline RNase Away & Invitrogen, USA \\
\hline SOC medium & Carl Roth, Germany \\
\hline Sodium chloride & Carl Roth, Germany \\
\hline
\end{tabular}




\begin{tabular}{|l|l|}
\hline Sodium citrate tribasictrihydrate & Carl Roth, Germany \\
\hline Sodium dodecylsulfate $(20 \% \mathrm{w} / \mathrm{v})$ & Carl Roth, Germany \\
\hline Sodium hydroxide & Carl Roth, Germany \\
\hline Stripping Buffer & Thermo Scientific, USA \\
\hline TOPRO-3 & Invitrogen, USA \\
\hline Trisbase & Sigma, USA \\
\hline Tris $1.5 \mathrm{M}(\mathrm{pH} 8.9)$ & Amresco, Germany \\
\hline Tris 0.5 M (pH 6.8) & Amresco, Germany \\
\hline Trizol reagent & Invitrogen, USA \\
\hline Tween 20 & Sigma, USA \\
\hline Xtreme gene siRNA transfection reagent & Roche, Germany \\
\hline Xylol & Carl Roth, Germany \\
\hline
\end{tabular}

\subsubsection{Kits}

\begin{tabular}{|l|l|}
\hline Names & Company \\
\hline BrdU Incoportation assay kit & Roche, Germany \\
\hline Dc Protein assay kit & BioRad, USA \\
\hline EndoFree plasmid maxiprep kit & Qiagen, USA \\
\hline InProm-II Reverse Transcriptase kit & Promega, USA \\
\hline In situ apoptosis & Roche, Germany \\
\hline NovaRed Substrate kit & Vector, USA \\
\hline RNeasy mini kit & Qiagen, USA \\
\hline $\begin{array}{l}\text { Supersignal west femto maximum sensitivity substrate } \\
\text { kit }\end{array}$ & Thermo Scientific, USA \\
\hline NovaRED substrate kit & Vector, USA \\
\hline
\end{tabular}

\subsubsection{Cell culture medium and reagents}

\begin{tabular}{|l|l|}
\hline Names & Company \\
\hline Dulbecco's Phosphate Bufffer saline (DPBS) & PAA, USA \\
\hline DBZ ( $\gamma$ secretase inhibitor) & AXON, Netherlands \\
\hline Endothelial growth medium (EGM-2) & Lonza, USA \\
\hline Fetal calf serum & PAA, USA \\
\hline OptiMEM-I+ GutaMax-I & Gibco, Germany \\
\hline Pencillin/Streptomycin & Lonza, USA \\
\hline Smooth muscle cell medium (SmGM) & Lonza, USA \\
\hline Trypsin/EDTA & Lonza, USA \\
\hline
\end{tabular}

\subsubsection{Equipments}

\begin{tabular}{|l|l|}
\hline Names & Company \\
\hline Agarose electrophoresis chambers & Biometra, USA \\
\hline BioDoc Analyzer & INTAS, Germany \\
\hline Cell culture incubator, Hera cell & Heraus, Germany \\
\hline
\end{tabular}




\begin{tabular}{|l|l|}
\hline Centrifuge & Thermoscientific \\
\hline CFX96 tm Real-Time PCR detection system & BioRad, USA \\
\hline Fluorescence microscope & Leica, USA \\
\hline Fujifim LAS 4000 & Fujifilm, Japan \\
\hline Light microscope & Hund, Germany \\
\hline Precellys Homogenizer & PeQLab, Germany \\
\hline Microplate reader Infinite 200 & TECAN, Germany \\
\hline PCR thermocycler & Eppendorf, USA \\
\hline Power supply & BioRad, USA \\
\hline Water bath (cell culture) & Heraus, Germany \\
\hline Western blot chambers & BioRad, USA \\
\hline
\end{tabular}

\subsubsection{Other materials}

\begin{tabular}{|l|l|}
\hline Names & Company \\
\hline Cell scrapers & BD Falcon, USA \\
\hline Cell culture dishes $(10 \mathrm{~cm}, 3 \mathrm{~cm}, 6$ well, 48well, 96well) & Griener bio-one, Germany \\
\hline Cell culture flasks $\left(75 \mathrm{~cm}^{2}, 25 \mathrm{~cm}^{2}\right)$ & Griener bio-one, Germany \\
\hline Centrifugal protein concentrators & Millipore, USA \\
\hline Filter tips $(10,100,1000 \mu \mathrm{l})$ & Griener bio-one, Germany \\
\hline Gel blotting paper & Whatman, USA \\
\hline Microcentrifuge tubes & Eppendorf, USA \\
\hline Nitroceullulose membrane & Pall Corporation, USA \\
\hline Polypropylene tubes $(15 \mathrm{ml}, 50 \mathrm{ml})$ & Griener bio-one, Germany \\
\hline Precellys Tubes with beads & PeQLab, Germany \\
\hline Real time PCR plates & BioRad, USA \\
\hline Tips $(10,100,1000 \mu l)$ & Eppendorf, USA \\
\hline Tissue culture chamber slides & BD Falcon, USA \\
\hline
\end{tabular}




\subsection{Methods}

\subsubsection{Animal experiments}

Animals (mice and rats) were purchased from Charles River Laboratories (Sulzfeld, Germany). All the experiments were performed in accordance with the National Institute of Health Guidelines on the Use of Laboratory Animals. Study protocols (Nr. B2/191, Nr. 40/2009 and GI 2010-Nr. 03/2009 and Nr. 80/2009) were approved by both, the University Animal Care Committee and the Federal Authorities for Animal Research of the Regierungspräsidium Giessen (Hessen, Germany).

\subsubsection{Monocrotaline (MCT) induced PH rat model}

The alkaloid MCT was dissolved in $1 \mathrm{~mol} / \mathrm{L} \mathrm{HCl}$ and then adjusted to $\mathrm{pH} 7.4$ with $1 \mathrm{~mol} / \mathrm{L}$ $\mathrm{NaOH}$, resulting in a clear solution with a final concentration of $20 \mathrm{mg} / \mathrm{ml}$. Adult male rats (250$300 \mathrm{~g}$ in body weight) were randomized and injected with a single subcutaneous (s.c.) injection of MCT $(60 \mathrm{mg} / \mathrm{kg}$ body weight) to induce pulmonary hypertension, or an injection of the same volume of saline as a control. Drinking water supplemented with antibiotic Baytril was given to MCT-injected rats for 2 weeks at the second day of MCT injection [48]. Hemodynamic studies and freezing of tissues were carried out 3 weeks, 4 weeks and 5 weeks after MCT injection.

\subsubsection{Hypoxia (Hox) induced PH mouse model}

C57BL/6 mice (8-week-old) were exposed to chronic hypoxia $\left(10 \% \mathrm{O}_{2}\right)$ in a ventilated chamber or kept under normoxic conditions as control, as described previously [48]. Hemodynamic studies and freezing of tissues were carried out after 3 weeks and 5 weeks of hypoxia exposure.

\subsubsection{Hypoxia+Su5416 induced PH rat model}

Su5416 (Semaxanib) was dissolved in DMSO at a concentration of $25 \mathrm{mg} / \mathrm{ml}$. Adult rats (200-250g in body weight) were randomized and injected with a single subcutaneous injection of freshly prepared Su5416 $(20 \mathrm{mg} / \mathrm{kg}$ body weight) in the area of neck. Animals were put into the ventilated hypoxia chamber $\left(10 \% \mathrm{O}_{2}\right)$ immediately after the injection. Saline injected control animals were kept in normoxic conditions for the same time period. Hemodynamic studies and freezing of tissues were carried out after 3 weeks of hypoxia exposure. 


\subsubsection{Hemodynamics measurement}

At the end of the treatment, the rats were anesthetized with an intraperitoneal injection (i.p.) of ketamine $(9 \mathrm{mg} / \mathrm{kg}$ body mass) and medetomidine $(100 \mu \mathrm{g} / \mathrm{kg}$ body mass), followed by an intramuscular (i.m.) injection of heparin (50lU/kg body mass) to measure the hemodynamic parameters. The rats were then tracheotomized and ventilated at a frequency of $60 \mathrm{breaths} / \mathrm{min}$, with a positive end expiratory pressure at $1 \mathrm{~cm} \mathrm{H}_{2} \mathrm{O}$ throughout. To measure right ventricular pressure, a right heart catheter (PE 50 tube) was inserted through the right jugular vein and to measure arterial pressure a polyethylene catheter was inserted into the left carotid artery.

\subsubsection{RNA isolation}

\subsubsection{RNA isolation from tissues}

Total RNA was isolated from lung homogenates using Qiagen RNeasy mini kit. 30mg of tissue was placed in $600 \mu \mathrm{l}$ of RLT buffer. Tubes were put into tissue homogenizer for 30 sec spins in three repeats. In between the spins, tubes were incubated on ice. After homogenization, samples were centrifuged at full speed for $3 \mathrm{~min}$. Supernatant was removed by pipetting into a new microcentrifuge tube. Equal volume of $70 \%$ ethanol was added to the lysate, mixed well and transferred to RNeasy spin column. Column was centrifuged at $10,000 \mathrm{rpm}$ for $15 \mathrm{sec}$ and flow through was discarded. $700 \mu$ l of buffer RW1 was added to the column, followed by centrifugation at $10,000 \mathrm{rpm}$ for $15 \mathrm{sec}$ and removal of flow through. To wash the column, $500 \mu \mathrm{l}$ of Buffer RPE was added to the column and centrifuged at 10,000rpm for 15sec. Flow through was discarded and the wash was repeated. For elution, column was placed in a new $1.5 \mathrm{ml}$ collection tube and $30 \mu \mathrm{l}$ of RNase free water was added directly to the spin column membrane, followed by centrifugation at $10,000 \mathrm{rpm}$ for $1 \mathrm{~min}$.

\subsubsection{RNA isolation from cells}

RNA isolation from primary hPAECs and hPASMCs was carried out using TRIzol ${ }^{\mathrm{TM}}$ reagent. Media was removed from the wells, followed by a PBS wash. $250 \mu$ l of TRIzol ${ }^{\mathrm{TM}}$ was directly added to each well of 6 well plates and after $2 \mathrm{~min}$ of incubation; lysates were scratched with cell scrapers and transferred to microcetrifuge tubes. $50 \mu \mathrm{l}$ of chloroform was added to each sample followed by vortexing, incubation for $10 \mathrm{~min}$ at RT and centrifugation at $4^{\circ} \mathrm{C}$ for $30 \mathrm{~min}$ at $12,000 \mathrm{~g}$. The upper aqueous phase was carefully transferred into a new tube, mixed with $125 \mu$ l of isopropanol, 
incubated at RT for $10 \mathrm{~min}$ and centrifuged for $20 \mathrm{~min}$ at $4^{\circ} \mathrm{C}$ at $12,000 \mathrm{~g}$. Supernatant was discarded and the precipitated RNA was washed with freshly prepared $75 \%$ ethanol and centrifuged for $5 \mathrm{~min}$ at $4^{\circ} \mathrm{C}$ at 7,500g. RNA pellet was dried for $10 \mathrm{~min}$ and dissolved in $12 \mu \mathrm{l}$ of RNase free water at $60^{\circ} \mathrm{C}$.

Nanoquant analyser was used to analyze the RNA concentration and purity. $1.5 \mu$ l of sample was applied to the nanoquant plate and spectroscopical absorance analysis was set as the ratio of $260 / 280 \mathrm{~nm}$ wavelengths. RNA samples with $260 / 280$ ratio around 1.8 were used for further experiments.

\subsubsection{Reverse transcription}

Reverse transcription refers to the enzymatic method of synthesizing complementary single stranded cDNA from mRNA with the help of a robust RNA dependent DNA polymerase, Reverse Transcriptase.

cDNA was synthesized from RNA isolated from different samples by two step PCR reaction using ImProm-IIM Reverse transcription system according to the manufacturer's instructions. In step I, template-primer mix consisting of $1 \mu \mathrm{g}$ of RNA and $1 \mu \mathrm{l}$ of oligo dT primer was incubated at $70^{\circ} \mathrm{C}$ for $5 \mathrm{~min}$ to allow denaturation of RNA, followed by incubation at $4^{\circ} \mathrm{C}$ for $5 \mathrm{~min}$. For step II, a reverse transcription mix was prepared on ice as follows:

\begin{tabular}{|c|c|c|}
\hline Components & $\begin{array}{c}\text { Final } \\
\text { Concentration }\end{array}$ & Volume (in $\mu \mathrm{l}$ ) \\
\hline InProm-II ${ }^{\mathrm{TM}} \mathbf{5 X}$ reaction buffer & $1 \mathrm{X}$ & 4 \\
\hline $\mathrm{MgCl} 2,25 \mathrm{mM}$ & $5 \mathrm{mM}$ & \\
\hline dNTP mix, 10mM & $0.5 \mathrm{mM}$ & 1 \\
\hline RNasin $^{\circledR}$ ribonuclease inhibitor & $0.5 U$ & 0.5 \\
\hline InProm-II ${ }^{\mathrm{TM}}$ reverse transcriptase & $1 U$ & 1 \\
\hline Nuclease free water (to a final volume of $15 \mu \mathrm{l}$ ) & & \\
\hline
\end{tabular}

Reverse transcription mix was added to the template-primer mix and was subjected to $25^{\circ} \mathrm{C}$ for $5 \mathrm{~min}$ to allow annealing, followed by an incubation at $42^{\circ} \mathrm{C}$ for $1 \mathrm{hr}$. Finally, the mix was subjected to $70^{\circ} \mathrm{C}$ for $15 \mathrm{~min}$ to allow thermal inactivation of the reverse transcriptase. cDNA product was stored at $-20^{\circ} \mathrm{C}$. 


\subsubsection{Quantitative real time PCR (qRT PCR)}

Real time PCR is a technique based on polymerase chain reaction that allows simultaneous amplification and quantification of target cDNA sequences by employing target specific primers. Quantity of the target sequence is expressed as a relative amount normalized to the housekeeping gene.

Intron spanning primers were designed using Primer-Blast program (NCBI) and are shown in Appendix Table 1. Product size was kept in between 100-250bp. Real time PCR was performed from the cDNA samples in $25 \mu$ l reactions using $\mathrm{iQ}^{\mathrm{TM}} \mathrm{SYBR}^{\circledR}$ Green Supermix (BioRad). The reaction was prepared as follows:

\begin{tabular}{lc}
\hline \multicolumn{1}{c}{ Component } & Volume added (in $\mu \mathrm{l})$ \\
\hline iQ $^{\mathrm{TM}}$ SYBR $^{\circledR}$ Green Supermix, 2X & 12.5 \\
Forward primer, $10 \mu \mathrm{M}$ & 0.5 \\
Reverse primer, $10 \mu \mathrm{M}$ & 0.5 \\
cDNA template & 2 \\
Nuclease free water & 9.5 \\
\hline
\end{tabular}

The reaction mix were pipetted into 96 well plate and centrifuged for $1 \mathrm{~min}$. qRT PCR was carried out in CFX96 ${ }^{\mathrm{TM}}$ Real-Time PCR detection system, using the following program:

\begin{tabular}{lccc}
\hline \multicolumn{1}{c}{ qRT PCR steps } & Temperature & Time & Number of repeats \\
\hline Initial denaturation & $95^{\circ} \mathrm{C}$ & 3 minutes & 1 \\
Denaturation & $95^{\circ} \mathrm{C}$ & $10 \mathrm{sec}$ & \\
Annealing & $58^{\circ} \mathrm{C} / 60^{\circ} \mathrm{C}$ & $30 \mathrm{sec}$ & 40 \\
Elongation & $72^{\circ} \mathrm{C}$ & $30 \mathrm{sec}$ & 1 \\
Denaturation & $95^{\circ} \mathrm{C}$ & $10 \mathrm{sec}$ & 1 \\
Melting curve & $65^{\circ} \mathrm{C}$ to $95^{\circ} \mathrm{C}$ & Increment $0,5^{\circ} \mathrm{C}$ for $5 \mathrm{sec}$ & \\
\hline
\end{tabular}

$*$ depending on the melting temperature of the primer set (Appendix Table 1 )

\subsubsection{Protein isolation}

\subsubsection{Protein isolation from tissues}

$100 \mathrm{mg}$ of tissue was weighed and put into $500 \mu \mathrm{l}$ of RIPA buffer (Thermo Scientific) containing protease and phosphatase inhibitor cocktail. Tissues were homogenized as described above (Section3.2.1.1), followed by centrifugation for $30 \mathrm{~min}$ at $4^{\circ} \mathrm{C}(13,000 \mathrm{xg})$. Supernatants were transferred to $1.5 \mathrm{ml}$ tubes and directly quantified or stored at $-80^{\circ} \mathrm{C}$. 


\subsubsection{Protein isolation from cells}

Protein isolation from primary hPAECs and hPASMCs was carried out using RIPA buffer (Thermo Scientific) containing protease and phosphatase inhibitor cocktail (Thermo Scientific). Media was removed from the wells, followed by a PBS wash. $75 \mu$ l of RIPA buffer was directly added to each well of 6 well plates and after $10 \mathrm{~min}$ of incubation at $4^{\circ} \mathrm{C}$; plates were scratched with cell scrapers and supernatants were put into $1.5 \mathrm{ml}$ tubes. The tubes were centrifuged for $30 \mathrm{~min}$ at $4^{\circ} \mathrm{C}(13,000 \mathrm{xg})$. Supernatants were transferred to $1.5 \mathrm{ml}$ tubes and directly quantified or stored at $-80^{\circ} \mathrm{C}$.

\subsubsection{Protein estimation}

Protein quantification was carried out using Bio-Rad DC Protein Assay kit. It is a colorimetric assay based on Lowry's method involving reaction of protein with an alkaline copper tartrate solution and Folin reagent giving rise to a characteristic blue color showing absorbance at $750 \mathrm{~nm}$. Different concentrations of BSA in range of $0.125-2 \mathrm{mg} / \mathrm{ml}$ were used as standard. Protein samples were prediluted in range of the standard and the absorbance was measured at 750nm using a microplate reader (TECAN). Exact concentrations were calculated depending on the standards.

\subsubsection{SDS polyacrylamide gel electrophoresis (SDS-PAGE)}

SDS PAGE is carried out to separate various proteins in a sample according to their molecular weights for further immunoblot analysis. Protein samples from cells and tissues were equalized to same concentrations and mixed with $5 \mathrm{X}$ gel loading buffer at a ratio of $4: 1$ and denatured at $95^{\circ} \mathrm{C}$ for $10 \mathrm{~min}$. Protein samples were loaded along with molecular weight marker into the wells of $7 \%$ or $10 \%$ (depending on protein sizes to be separated) polyacrylamide gels. Gels were run in vertical electrophoretic assembly using $1 \mathrm{X}$ running buffer at $100-120 \mathrm{~V}$ for $2-3 \mathrm{hrs}$. Buffers used are as follows:

\begin{tabular}{lc}
\hline $\mathbf{5 X}$ gel loading buffer components & Final concentration \\
\hline Tris- $\mathrm{HCl}(\mathbf{2 M}, \mathrm{pH}-6.8)$ & $375 \mathrm{mM}$ \\
SDS & $10 \%(\mathrm{w} / \mathrm{v})$ \\
Glycerol & $50 \%(\mathrm{v} / \mathrm{v})$ \\
$\boldsymbol{\beta}-$ Mercaptoethanol & $12.5 \%(\mathrm{v} / \mathrm{v})$ \\
Bromophenol Blue & $0.02 \%(\mathrm{w} / \mathrm{v})$ \\
\hline
\end{tabular}




\begin{tabular}{lc}
\hline 1X SDS Running buffer components & Final concentration \\
\hline Tris & $25 \mathrm{mM}$ \\
Glycine & $250 \mathrm{mM}$ \\
SDS $10 \%(w / v)$ & $0.1 \%(w / v)$ \\
\hline
\end{tabular}

Stacking gel (5\%)

\begin{tabular}{lc}
\hline Gel Component & Final Concentration \\
\hline Tris-HCl (0.5M, pH-6.8) & $125 \mathrm{mM}$ \\
Acrylamide/Bis-acrylamide & $6 \%$ \\
SDS 10\% (w/v) & $0.10 \%$ \\
APS 10\% (w/v) & $0.05 \%$ \\
TEMED & $0.10 \%$ \\
Water upto the final volume & \\
\hline
\end{tabular}

\section{Resolving gel}

\begin{tabular}{lcc}
\hline Gel component & \multicolumn{2}{c}{ Percentage of gel } \\
\hline & $7 \%$ & $10 \%$ \\
Tris-HCl (1.5M, pH-8.8) & $375 \mathrm{mM}$ & $375 \mathrm{mM}$ \\
Acrylamide/Bis-acrylamide & $7 \%$ & $10 \%$ \\
SDS 10\% (w/v) & $0.10 \%$ & $0.10 \%$ \\
APS 10\% (w/v) & $0.05 \%$ & $0.05 \%$ \\
TEMED & $0.10 \%$ & $0.10 \%$ \\
Water upto the final volume & & \\
\hline
\end{tabular}

\subsubsection{Immunoblotting}

Proteins separated on gel were transferred to a nitrocellulose membrane by electrophoretic transfer. The transfer was carried out for $1 \mathrm{hr}$ at $100 \mathrm{~V}$ in transfer buffer.

\begin{tabular}{lc}
\hline Blotting buffer components & Final concentration \\
\hline Tris & $25 \mathrm{mM}$ \\
Glycine & $192 \mathrm{mM}$ \\
Methanol & $20 \%(\mathrm{v} / \mathrm{v})$ \\
\hline
\end{tabular}

After the transfer, membranes were removed and blocked in blocking buffer for $1 \mathrm{hr}$ on shaker at RT, followed by overnight incubation in primary antibodies diluted in blocking buffer (for dilutions, Appendix Table 2) at $4^{\circ} \mathrm{C}$. Following day, membranes were washed 3 times for $10 \mathrm{~min}$ with 1xTBST buffer and subsequently incubated in secondary HRP-conjugated antibodies diluted in blocking buffer for $1 \mathrm{hr}$ at RT. After $1 \mathrm{hr}$ of incubation, membranes were washed thrice for $10 \mathrm{~min}$ in 1x TBST and incubated with ECL substrate (Thermo Scientific) in the Image reader (Fujifilm) to detect the signal. The time of exposure was determined on the basis of signal intensity. 
Tris buffer saline (TBS) components

\section{Tris}

Sodium chloride

Tween 20

$\mathrm{HCl}$

Water
Final Concentration

\section{$20 \mathrm{mM}$}

$137 \mathrm{mM}$

$0.1 \%(\mathrm{v} / \mathrm{v})$

to set the $\mathrm{pH}$ to 7.4

Upto final volume

Blocking buffer (5\% non-fat dry milk in 1xTBST)

In order to re-probe the membranes for housekeeping genes, membranes were stripped in a stripping buffer (Thermo Scientific) for $30 \mathrm{~min}$ at $37^{\circ} \mathrm{C}$, washed in TBST and probed again with primary antibody.

\subsubsection{Densitometric analysis of the immunoblots}

Western blots were quantified using the multi gauge software (Fujifilm). Expression was quantified using bands intensity values (in arbitrary units), which were normalized to the housekeeping genes (GAPDH or $\beta$-actin).

\subsubsection{Immunohistochemistry}

$4 \mu \mathrm{m}$ thick sections were cut from paraffin embedded lung tissues. Sections were incubated at $65^{\circ} \mathrm{C}$ for $20 \mathrm{~min}$, followed by deparaffinization in xylene and rehydration in series of gradedecreasing ethanol solutions. Sections were washed with PBS and incubated in boiling 10mM citrate buffer for $8 \mathrm{~min}$ at 630 watt in microwave for antigen retrieval. When needed, antigen retrieval was carried out by using $0.25 \%$ trypsin for $10 \mathrm{~min}$ at $37^{\circ} \mathrm{C}$. After blocking endogenous peroxidases activity by treatment with $15 \%$ hydrogen peroxide for $20 \mathrm{~min}$, NovaRED horseradish peroxidase (HRP)-substrate kit was used for immunohisotchemical staining according to the manufacturer's instructions. Sections were kept in serum blocking for $1 \mathrm{hr}$, followed by overnight incubation with primary antibody at $4^{\circ} \mathrm{C}$. After washing with PBS, sections were incubated with biotinylated secondary antibody for $10 \mathrm{~min}$, followed by again a PBS wash and incubation with streptavidin conjugated HRP for $5 \mathrm{~min}$. Sections were washed and color development was carried out using a substrate/chromogen mixture, followed by counterstaining with hematoxylin. Stained sections were examined under Leica DM 2500 microscope using Leica QWin imaging software. 


\subsubsection{BrdU incorporation assay}

Cellular proliferation for hPASMCs and hPAECs was determined using colorimetric BrdU incorporation assay kit (Roche) according to manufacturer's instructions. The kit is based on the principle of detecting Br-deoxycitidine incorporated into DNA during DNA synthesis by using antiBrdU antibody conjugated with peroxidase enzyme. Rate of DNA synthesis is taken as a marker for cellular proliferation.

After defined period of culture, transiently transfected or inhibitor treated cells in 48well plate or 96well plate respectively, were incubated with BrdU labeling solution for $2 \mathrm{hrs}$. After the $2 \mathrm{hr}$ incubation, wells were washed with PBS and subsequently fixed with FixDenat solution for $30 \mathrm{~min}$. Fixed cells were then incubated with anti-BrdU-POD antibody for 90min, washed three times with PBS and further incubated with substrate solution until color development. Absorbance was measured at $370 \mathrm{~nm}$ with reference at $492 \mathrm{~nm}$ in ELISA plate reader (TECAN). Proliferation of cells was plotted as a function of absorbance at $370 \mathrm{~nm}$.

\subsubsection{Transformation and plasmid isolation}

In order to amplify the plasmids, they were transformed into competent bacterial cells by heat shock method and isolated using endofree Plasmid maxiprep kit (Qiagen).

$100 \mu \mathrm{l}$ aliquots of chemically competent XL-blue strain of E.coli cells were thawed on ice. To each aliquot, 20-50ng of plasmid DNA was added, mixed by gentle flicking and incubated on ice for $5 \mathrm{~min}$, followed by a heat shock at $42^{\circ} \mathrm{C}$ for $90 \mathrm{sec}$. Cells were placed on ice for $2 \mathrm{~min}$ and then incubated at $37^{\circ} \mathrm{C}$ for $1 \mathrm{hr}$ with $900 \mu \mathrm{l}$ of SOC medium. $100 \mu \mathrm{l}$ of the transformation mix was spread on LB agar plates containing ampicillin suitable for selecting transformed bacteria. Plates were incubated for $16 \mathrm{hrs}$ at $37^{\circ} \mathrm{C}$.

For plasmid isolation, single bacterial colony was picked from the LB agar plate with a sterile pipette tip and inoculated in $5 \mathrm{ml}$ of LB medium with ampicillin for $8 \mathrm{hrs}$ at $37^{\circ} \mathrm{C} .100 \mu \mathrm{l}$ of this culture was further inoculated in $250 \mathrm{ml}$ of ampicillin containing LB medium, grown at $37^{\circ} \mathrm{C}$ for $16 \mathrm{hrs}$ with shaking. Bacterial cells were harvested by centrifuging at $4500 \mathrm{rpm}$ for $20 \mathrm{~min}$ at $4^{\circ} \mathrm{C}$. Plasmids were isolated using Endo-free Plasmid Maxi kit, according to manufacturer's protocol.

Plasmid yield and purity were measured by Nanodrop and stored at $-20^{\circ} \mathrm{C}$ until further experiments. 


\subsubsection{Cell culture}

All the cell culture experiments were carried out in human pulmonary artery -smooth muscle cells (hPASMCs) and -endothelial cells (hPAECs). Cells were maintained at $37^{\circ} \mathrm{C}$ and $5 \% \mathrm{O}_{2}$ in humidified chamber and cultured in the medium provided from the company (EGM-2 for hPAECs and SmGM for hPASMCs). Cells were allowed to grow until $90-95 \%$ confluency and then subcultured. For, subculturing, cells were washed twice with DPBS and incubated with Trypsin/EDTA for 4 mins. Trypsin/EDTA was neutralized by addition of equal volume of FCS. Cells were centrifuged, resuspended in normal growth medium, counted and seeded into new culture dishes.

For freezing the cells, after trypsinization, cells were resuspended in FCS with 5\% DMSO. Then the cells were aliquoted in $1 \mathrm{ml}$ cryovials, which were subsequently frozen in an isopropanol box in $-80^{\circ} \mathrm{C}$. Next day, the cryovials were transferred to liquid nitrogen. When needed, cryovials containing the cells were thawed quickly at $37^{\circ} \mathrm{C}$ and seeded into a new culture dish. The following day, medium was changed to remove traces of DMSO.

\subsubsection{Transfection with over expression plasmid}

Transfection refers to the process of introducing foreign DNA or RNA into the mammalian cells. In this study, lipofection was used as the method of transfection. Lipofection refers to use of synthetic cationic lipid to facilitate the delivery of DNA into the cells. These cationic lipids tend to form liposomes in aqueous solution. Liposomes, thus formed, interact with negatively charged DNA to form lipid-DNA complexes. The complex fuses with the plasma membrane of cells, resulting in the uptake of DNA and further expression of the gene carried by the plasmid DNA.

hPAECs and hPASMCs were cultured to $70-80 \%$ confluence in 6 well plates (for protein isolation) or 48 well plates (for proliferation assay). Plasmid and lipofectamine were diluted in antibiotic free opti-MEM medium and mixed within $5 \mathrm{~min}$ at ratio of 1:2 (DNA in $\mu \mathrm{g}$ to transfection reagent in $\mu l$ ). The mixture was incubated at RT for $30 \mathrm{~min}$ and added to the cells that were cultured in antibiotic free transfection medium (opti-MEM medium + basal growth medium with $0.1 \%$ FCS). After $5 \mathrm{hrs}$, medium was changed to the normal growth medium upto $48 \mathrm{hrs}$, followed by protein isolation or proliferation assay. 


\subsubsection{RNA interference}

Cells were transfected with siRNA using X-tremeGENE siRNA transfection reagent (Roche) according to manufacturer's protocol. hPAECs and hPASMCs were cultured to $50-60 \%$ confluence. siRNA and transfection reagent were diluted in antibiotic free opti-MEM medium and mixed within 5 min at ratio of 1:5 (siRNA in $\mu \mathrm{g}$ to transfection reagent in $\mu$ ). The mixture was incubated at RT for $20 \mathrm{~min}$ and added to the cells that were cultured in antibiotic free transfection medium (opti-MEM medium + basal growth medium with $0.1 \%$ FCS). After 5hrs, medium was changed to the normal growth medium after $48 \mathrm{hrs}$, followed by protein isolation, proliferation assay or apoptosis assay.

\subsubsection{In vitro apoptosis assay}

hPAECs were assessed for apoptosis using In Situ cell death detection kit (Roche) according to manufacturer's instructions. The method is based on labeling double stranded DNA breaks (hallmark of cellular apoptosis) in individual cells by using terminal transferase (TdT), which incorporates fluorescein dUTP at 3' OH ends of genomic DNA.

hPAECs were grown in 8 well chamber slides, transfected with siRNA and 48hrs later, washed and fixed with $4 \%$ para formaldehyde for $1 \mathrm{hr}$. Fixed cells were permeabilized by adding $0.1 \%$ sodium citrate solution for $2 \mathrm{~min}$ on ice and incubated with labeling solution for $1 \mathrm{hr}$ at $37^{\circ} \mathrm{C}$. Slides were washed thrice with PBS, incubated with nuclear stain, Draq5 for $10 \mathrm{~min}$ and mounted with anti-fade mounting medium. Slides were analyzed for fluorescence using Leica fluorescence microscope.

\subsubsection{7 $\mathrm{Bax} / \mathrm{Bcl}-2$ ratio}

The ratio of Bax (an apoptosis promoter) to $\mathrm{Bcl}-2$ (an apoptosis inhibitor) protein is used widely to determine the susceptibility of cells towards apoptosis, under given conditions. Higher ratio is indicative of increased apoptosis while lower ratio is indicative of cell survival. In order, to determine the ratio, western blots were carried out using antibodies against Bax and $\mathrm{Bcl}-2$ proteins.

Standardization of protein loading was achieved as follows: (i) protein measurements of all samples were performed using the DcBioRad assay as described above (section 3.2.1.1) and equal

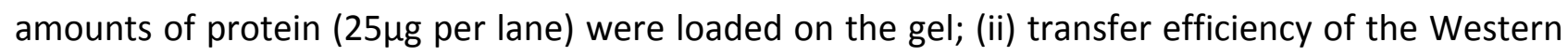


blots was checked by staining the membranes with $0.5 \%$ Ponceau Red in $1 \%$ acetic acid; (iii) detection of Bax and $\mathrm{Bcl}-2$ was performed separately on the same membrane. Thus, this procedure is valid to determine the protein ratios in the same sample (on the same lane of the gel). Quantitative analysis of the Bax and $\mathrm{Bcl}-2$ protein expression was performed using multi gauge software (Fujifilm) and band density values are expressed in arbitary units (A.U.).

\subsubsection{Statistical analysis}

All data are expressed as Mean \pm standard error of mean (SEM). Statistical comparisons of samples were performed by Student's t test for comparing two groups or by one-way ANOVA followed by the Newman-Keuls post-hoc test for multiple comparisons. Difference with $p<0.05$ between the groups was considered significant. 


\section{RESULTS}

\subsection{Regulation of Notch receptors and ligands in monocrotaline (MCT) induced PH rats}

\subsubsection{Hemodynamics and right heart hypertrophy in MCT rats}

Increase in right ventricular systolic pressure (RVSP) and ratio of right ventricular to left ventricular plus septal weight $(\mathrm{RV} / \mathrm{LV}+\mathrm{S})$ are taken as index of severity of pulmonary hypertension. Monocrotaline (MCT) treatment led to a marked increase in RVSP after 3 weeks $(42.3 \pm 5.33$ $\mathrm{mmHg}), 4$ weeks $(81.1 \pm 9.90 \mathrm{mmHg})$ and 5 weeks $(88.7 \pm 7.76 \mathrm{mmHg})$, when compared to saline injected control animals $(28.3 \pm 2.16 \mathrm{mmHg}$ ) (Figure $4.1 \mathrm{~A})$ without a significant difference in systemic arterial pressure (SAP) (Figure 4.1B). The ratio of right ventricular to left ventricular plus septal weight $(R V / L V+S)$, was significantly increased after $M C T$ administration from $0.29 \pm 0.01$ in control animals to $0.63 \pm 0.04$ in 5 weeks MCT rats (Figure $4.1 \mathrm{C}$ ).

A)

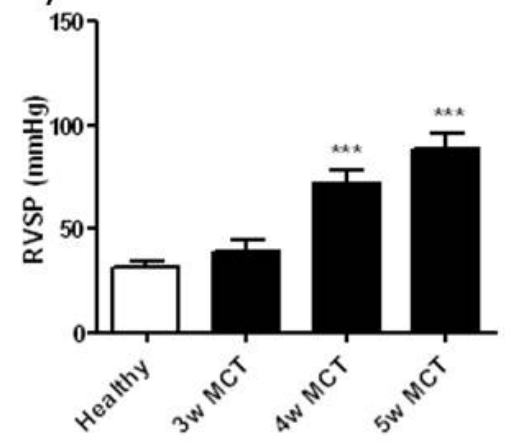

B)

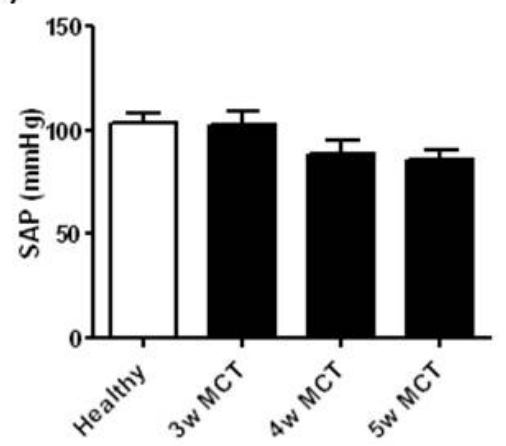

C)

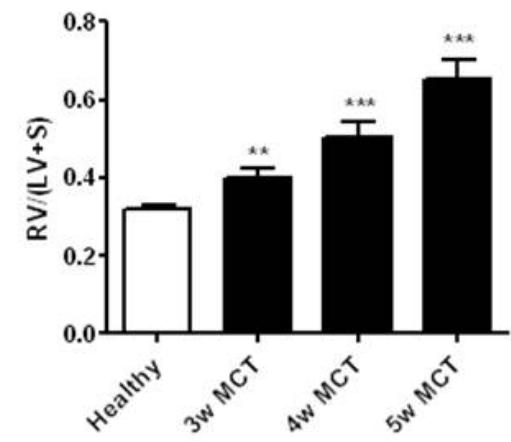

Figure 4.1 Hemodynamic measurements and right heart hypertrophy in Monocrotaline induced PH rats. Physiological measurements were taken 3 weeks ( 3 w MCT), 4 weeks (4w MCT) and 5 weeks (5w MCT) after MCT injection (A) Right ventricular systolic pressure (RVSP), (B) measurement of RV hypertrophy $[R V /(L V+S)]$ and (C) systemic arterial pressure (SAP). ${ }^{*} p<0.05,{ }^{* *} p<0.01, * * * p<0.001$ Vs Healthy, $(n=6)$.

\subsection{2 mRNA expression of Notch receptors and ligands in lung homogenates}

The mRNA expression profile of canonical Notch signaling pathway receptors (Notch1, 2, 3 and 4) and ligands (Jagged1 and 2, DLL1, 3 and 4) was analyzed in lungs from MCT induced PH rats after 3-, 4- and 5- weeks of MCT injection as well as in healthy rats. Among Notch receptors, Notch1 and Notch4 showed a marked decrease in mRNA expression in lungs from 3-, 4- and 5weeks MCT treated rats as compared to the control lungs while Notch3 exhibited an increase in MCT rats (Figure 4.2A). Out of the five ligands, only DLL4 exhibited a strong downregulation in mRNA expression in MCT lungs (Figure 4.2B). Other receptors and ligands did not show significant changes (data not shown). 
A)
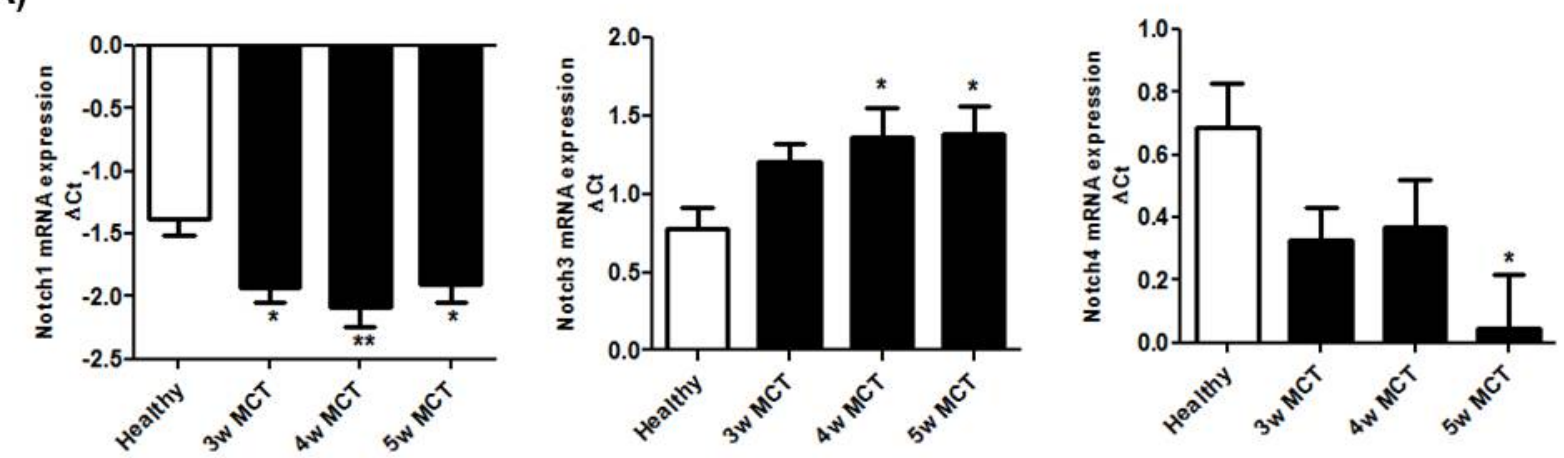

B)
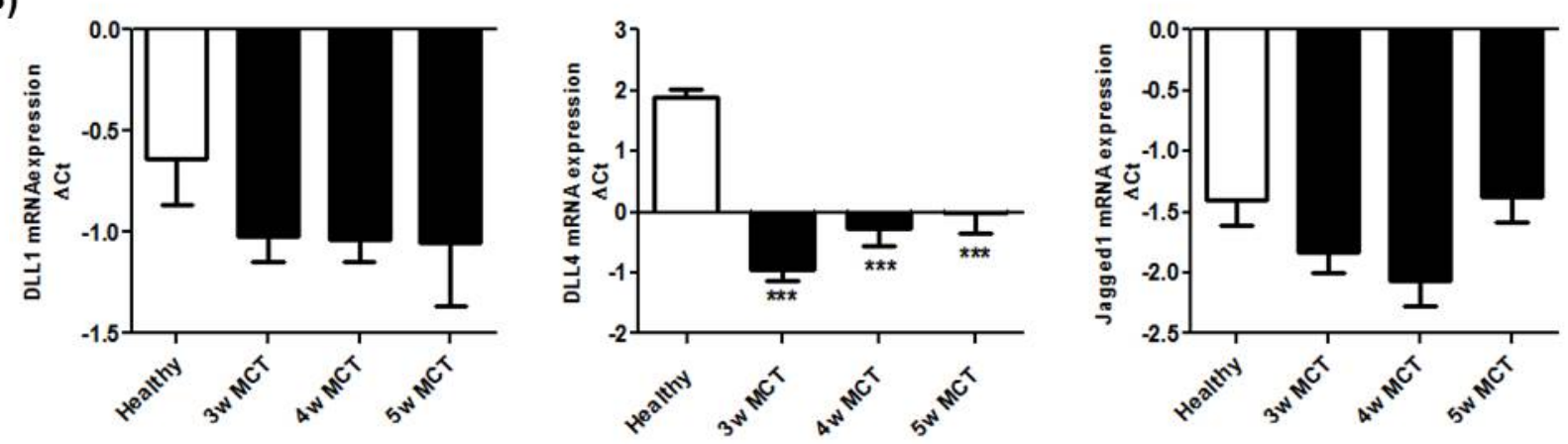

Figure 4.2 mRNA expression of Notch receptors and ligands in lung homogenates of Monocrotaline (MCT) induced PH rats. mRNA expression of Notch (A) receptors and (B) ligands in MCT induced PH rat lungs after 3 weeks ( 3 w MCT), 4 weeks ( 4 w MCT) and 5 weeks ( 5 w MCT) of MCT injection and healthy animals. Regulation was analyzed by real-time PCR with the Ct method $(n=5) .{ }^{*} p<0.05,{ }^{* *} p<0.01,{ }^{* * *} p<0.001$ Vs Healthy.

\subsubsection{Protein expression of Notch receptors and ligands in lung homogenates}

Protein expression profile was further investigated in the lungs of MCT rats 3-, 4- and 5weeks after MCT injection and compared with healthy rat lungs. Western blot analysis followed by quantification (normalized to GAPDH) demonstrated a marked decrease in protein levels of Notch receptors 1, 3 and 4 (Figure 4.3A and C) and ligands; DLL1, DLL4 and Jagged1 (Figure 4.3B and D) in MCT rat lungs as compared to healthy rat lungs. 

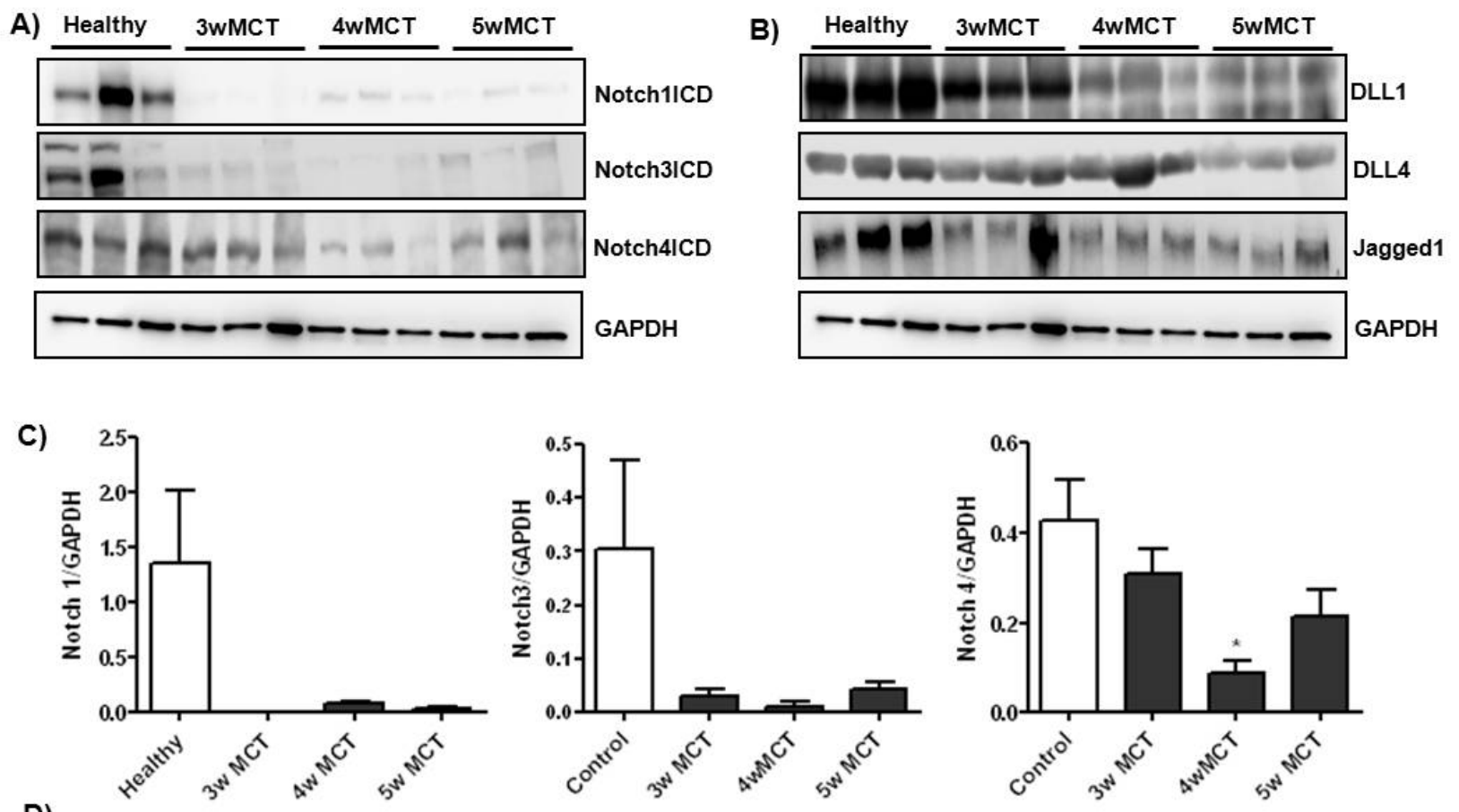

D)
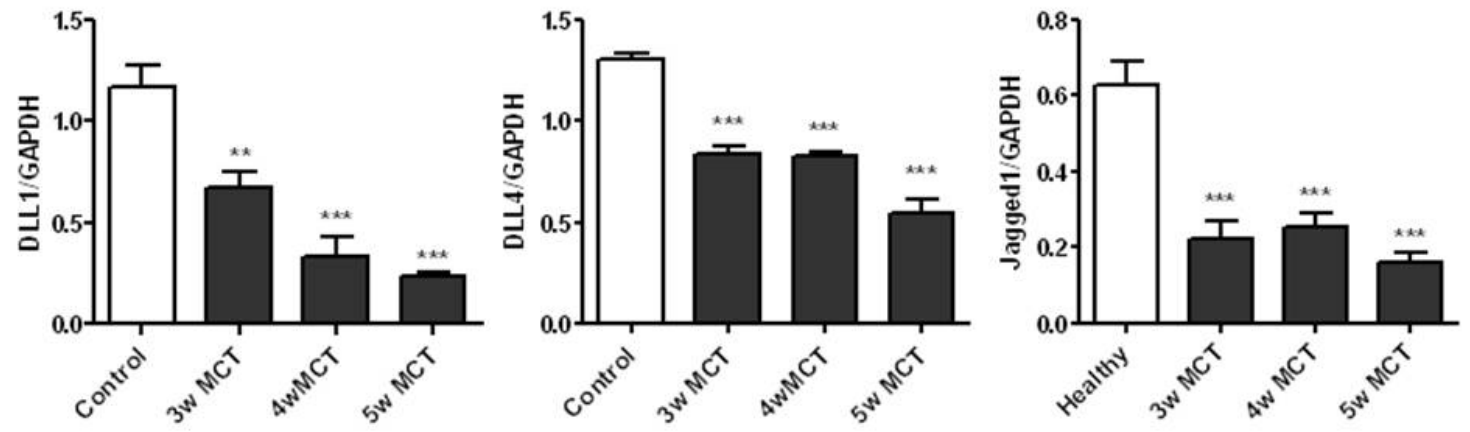

Figure 4.3 Protein expression of Notch receptors and ligands in lung homogenates of Monocrotaline (MCT) induced PH rats. Western blot analysis of Notch (A) receptors and (B) ligands in MCT induced PH rat lungs after 3 weeks ( 3 w MCT), 4 weeks ( 4 w MCT) and 5 weeks (5w MCT) of MCT injection and healthy animals, followed by the densitometry. Quantification of the immunoblots for (C) Notch receptors and (D) Notch ligands. GAPDH was taken as the loading control. ${ }^{*} p<0.05,{ }^{* *} p<0.01,{ }^{* * *} p<0.001$ Vs Healthy.

\subsection{Regulation of Notch receptors and ligands in hypoxia (Hox) induced PH mice}

\subsubsection{Hemodynamics and right heart hypertrophy in Hox mice}

Hypoxia exposure caused a marked increase in right ventricular systolic pressure after 3 weeks $(33 \pm 1.29 \mathrm{mmHg}$ ) and 5 weeks $(35 \pm 0.91 \mathrm{mmHg})$, when compared to normoxic mice $(26 \pm 0.40$ $\mathrm{mmHg}$ (Figure 4.4A). The ratio of right ventricular to left ventricular plus septal weight (RV/LV+S), was significantly increased after hypoxia exposure from $0.23 \pm 0.13$ in control animals to $0.36 \pm 0.014$ in 5 weeks hypoxic mice (Figure 4.4B). No significant changes were observed systemic arterial pressure (Figure 4.4C). 
A)

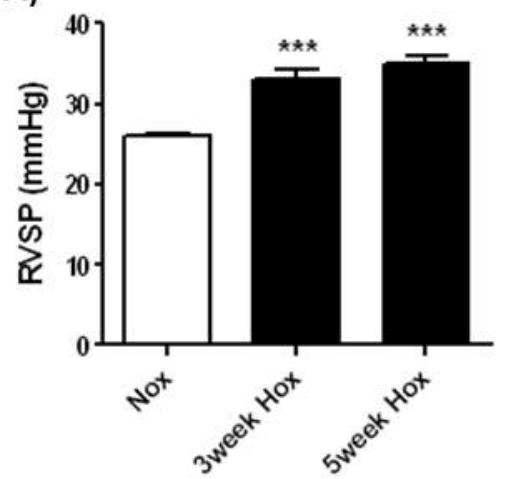

B)

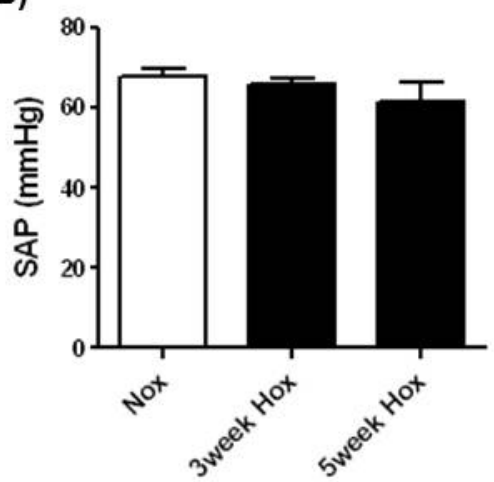

C)

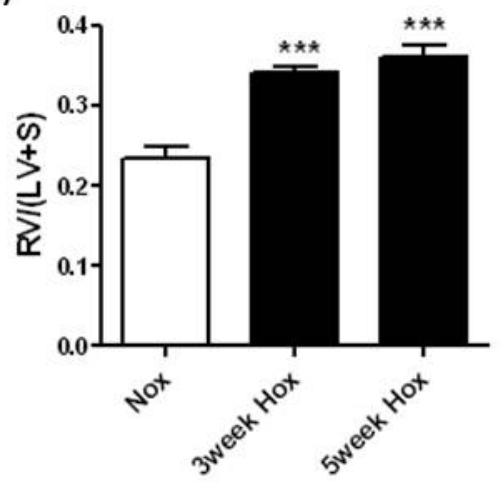

Figure 4.4 Hemodynamic measurements and right heart hypertrophy in Hypoxia (HOX) induced PH mice. Physiological measurements were taken 3 weeks ( 3 week Hox) and 5 weeks ( 5 w Hox) of hypoxia exposure. (A) Right ventricular systolic pressure (RVSP), (B) systemic arterial pressure (SAP) and (C). measurement of RV hypertrophy $[\mathrm{RV} /(\mathrm{LV}+\mathrm{S})] * \mathrm{p}<0.05, * * \mathrm{p}<0.01, * * * \mathrm{p}<0.001$ Vs NOX, $(\mathrm{n}=6)$.

\subsection{2 mRNA expression of Notch receptors and ligands in lung homogenates}

Expression of Notch receptors and ligands was investigated by qRT-PCR in lungs from mice exposed to hypoxia for 3- and 5-weeks and normoxic mice. Notch3 expression was increased and DLL4 expression was decreased significantly in 3- and 5-weeks hypoxia exposed mice lungs (Figure 4.5A) in comparison with normoxic mice. Apart, no significant changes were observed in the expression of other receptors and ligands (data not shown).

A)
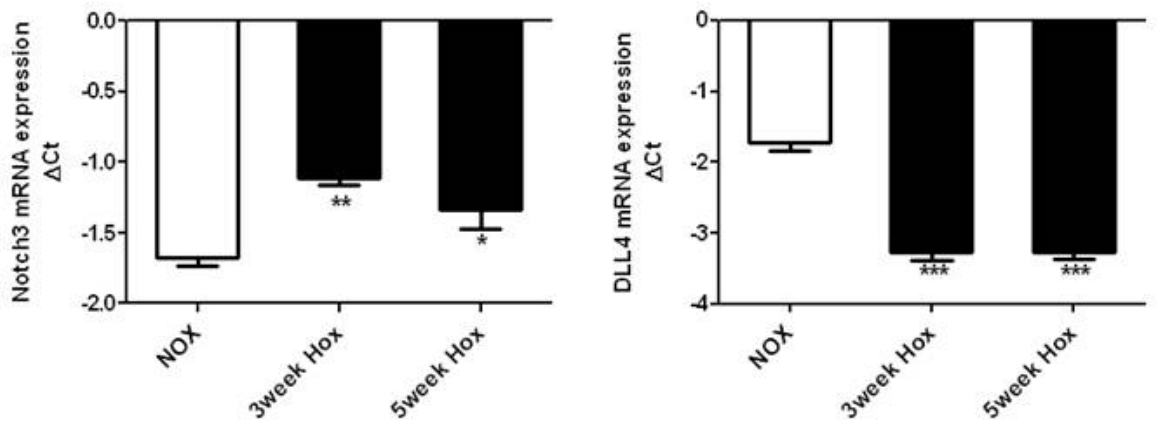

B)

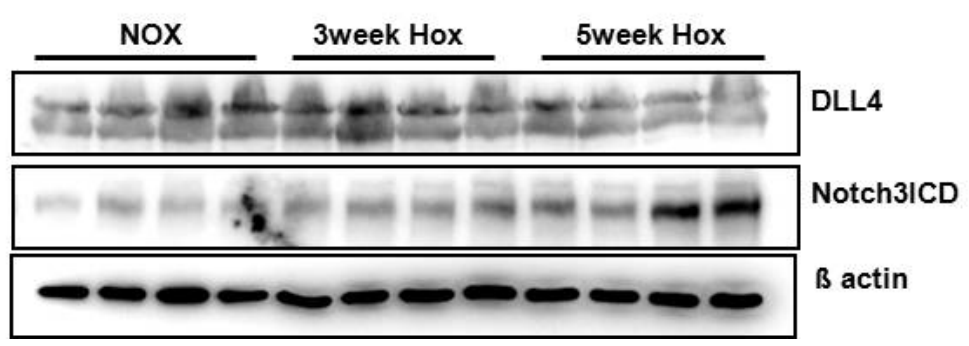


C)
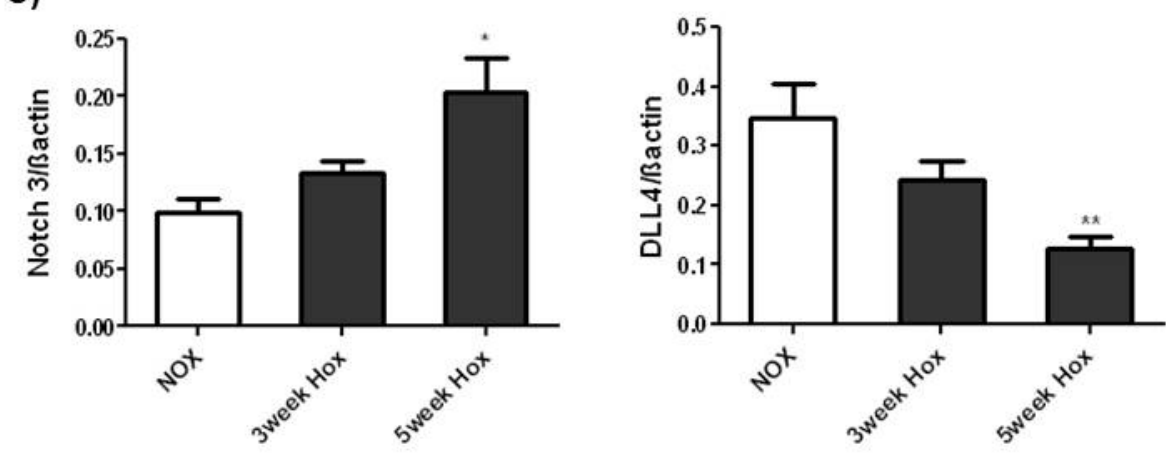

Figure 4.5 Expression of Notch receptors and ligands in lung homogenates of Hox induced PH mice. (A) mRNA expression of Notch3 and DLL4 in lung homogenates of Hypoxia after 3 weeks (3 week Hox) and 5 weeks ( 5 week Hox) of hypoxia exposure and healthy normoxic (NOX) mice. Regulation at mRNA level was analyzed by real-time PCR with the Ct method $(n=4)$. (B) Protein levels of Noth3ICD and DLL4 as analyzed by western blotting, followed by (C) densitometric analysis of the blots. ßactin was taken as the loading control. ${ }^{*} p<0.05, * * p<0.01,{ }^{* * *} p<0.001$ Vs NOX.

\subsubsection{Protein expression of Notch receptors and ligands in lung homogenates}

In agreement with the mRNA expression, Notch3ICD protein levels were increased while DLL4 levels were decreased in Hox mice in comparison with NOX mice (Figure 4.5B and C).

\subsection{Regulation of Notch receptors and ligands in Hypoxia+Su5416 (Hox+Su5416) induced PAH rats}

\subsubsection{Hemodynamics and right heart hypertrophy in Hox+Su5416 rats}

Compared with saline injected normoxia exposed rats $(35.0 \pm 0.84 \mathrm{~mm} \mathrm{Hg})$, Su5416 injection, followed by hypoxia exposure resulted in a marked increase in right ventricular systolic pressure $(65.0 \pm 2.32 \mathrm{~mm} \mathrm{Hg}$ ) (Figure 4.6A) without a significant difference in systemic arterial pressure (Figure 4.6B). The RV hypertrophy was significantly increased in Hox+Su5416 rats $(0.23 \pm 0.004$ to $0.60 \pm 0.01)$.
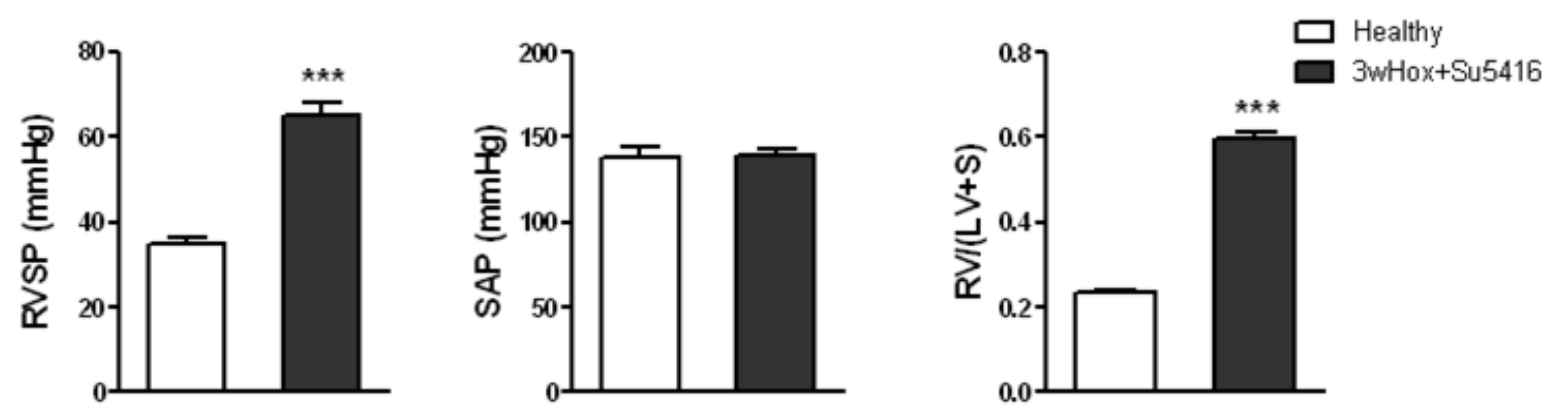
Figure 4.6 Hemodynamic measurements and right heart hypertrophy in Hypoxia+Su5416 induced PH rats. Physiological measurements were taken after 3 weeks (3wHox+Su5416) of hypoxia exposure. (A) Right ventricular systolic pressure (RVSP), (B) systemic arterial pressure (SAP) and (C) measurement of RV hypertrophy $[R V /(L V+S)] * p<0.05, * * p<0.01, * * * p<0.001$ Vs Healthy $(n=6)$.

\subsection{2 mRNA expression of Notch receptors and ligands in lung homogenates}

Expression of Notch receptors and ligands was investigated by qRT-PCR in lungs from rats exposed to hypoxia for 3 weeks after Su5416 injection and healthy rats. Notch1, Notch3, Notch4, Jagged1 and Jagged2 were strongly upregulated while DLL4 was downregulated in Hox+Su5416 rats as compared to healthy rats (Figure 4.7A).

\subsubsection{Protein expression of Notch receptors and ligands in lung homogenates}

Western blot analysis of Notch receptors and ligands revealed a marked increase in Notch1ICD and Notch3ICD protein levels in lungs from Hox+Su5416 rats in comparison to healthy rats (Figure 4.7B-C). Other receptors and ligands did not exhibit any significant changes (data not shown).

A)

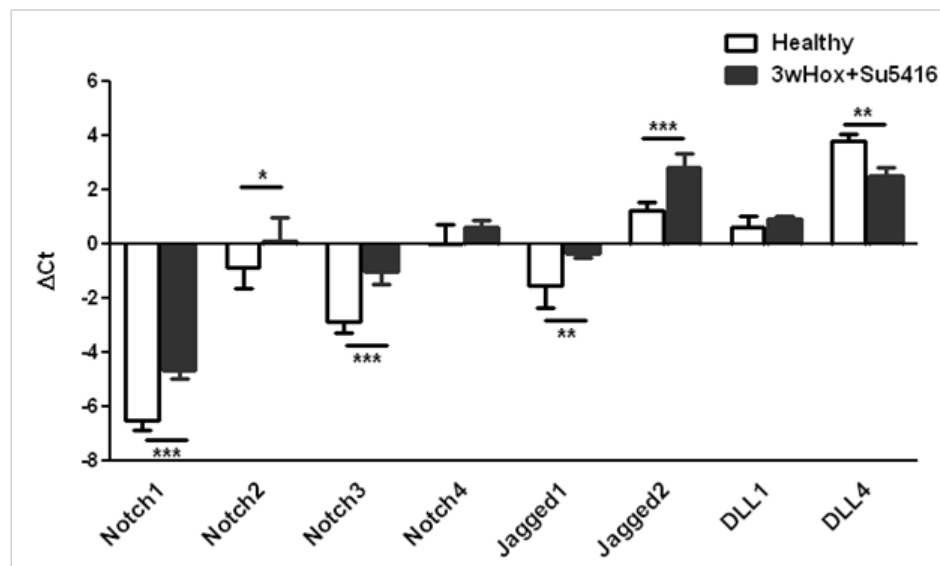

B)

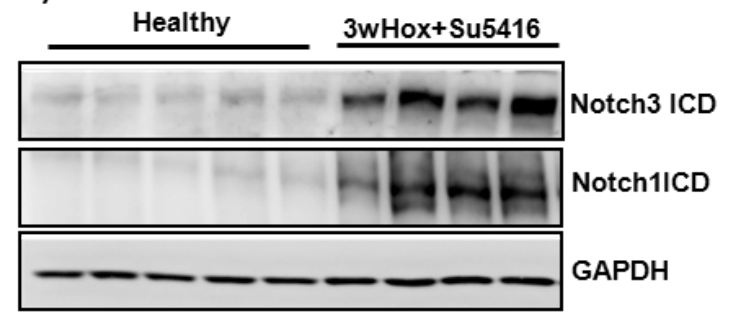

C)

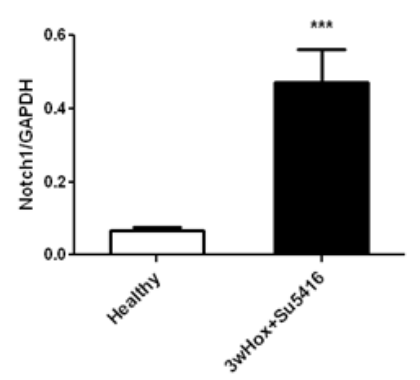

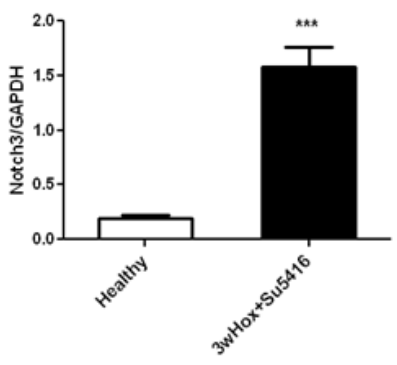

Figure 4.7 Expression of Notch receptors and ligands in Hypoxia+Su5416 rats lungs. (A) mRNA expression of Notch receptors and ligands in lung homogenates of Hypoxia+Su5416 rats after 3 weeks (3wHox+Su5416) of hypoxia exposure and control healthy rats. Regulation at mRNA level was analyzed by 
real-time PCR with the Ct method ( $n=6$ ) (B) Protein levels of Noth1ICD and Notch3ICD as analyzed by western blotting, followed by (C) densitometric analysis of the blots. GAPDH was taken as the loading control. ${ }^{*} p<0.005, * * p<0.01, * * * p<0.001$ Vs Healthy.

\subsection{Regulation of Notch receptors and ligands in IPAH patients}

\subsection{1 mRNA expression of Notch receptors and ligands in lung homogenates}

In order to define the mRNA expression of Notch receptors and ligands in lung homogenates from donors and patients with IPAH, qRT PCR was carried out. Among the Notch receptors, Notch 1, 3 and 4 were significantly upregulated in IPAH lungs. Among the ligands, Jagged 1 and 2 were increased in IPAH lungs as compared to Donor lungs (Figure 4.9A).

\subsubsection{Protein expression of Notch receptors and ligands in lung homogenates}

Protein expression analysis of Notch receptors and ligands in lung homogenates from patients with IPAH and donors revealed a significant increase in Notch1ICD levels in IPAH lungs, compared to donors as observed by western blot analysis (Figure 4.9B-C). Other receptors and ligands did not show significant changes (data not shown).

A)

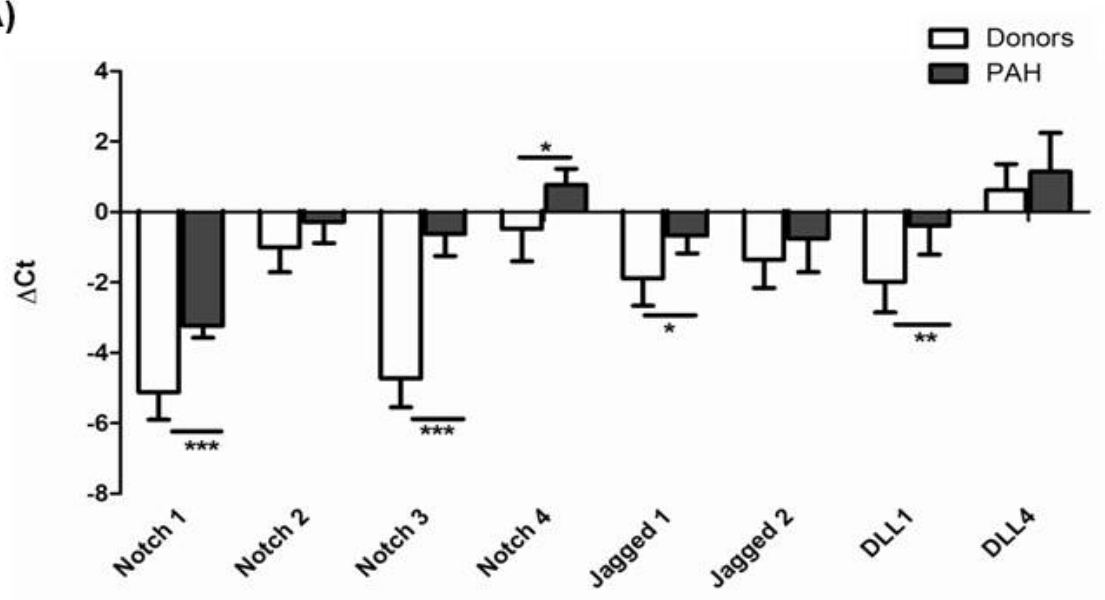


B)

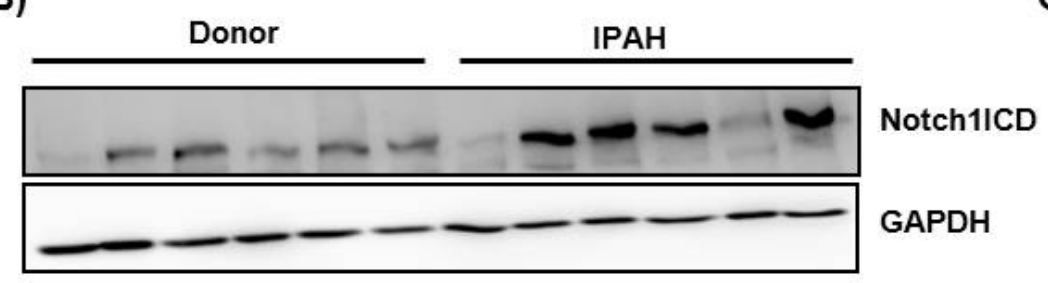

C)

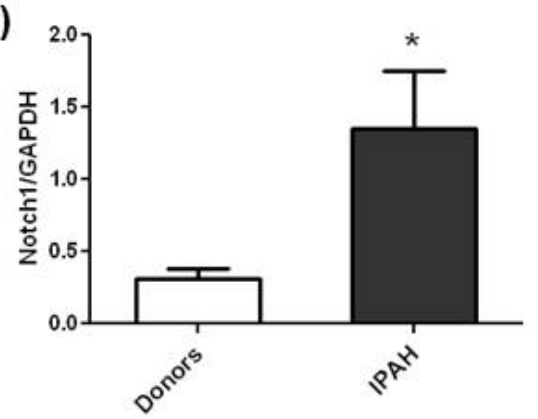

Figure 4.8 Expression of Notch receptors and ligands in human donors and IPAH lungs. (A) mRNA expression of Notch receptors and ligands in lung homogenates of donors and IPAH patients. Regulation at mRNA level was analyzed by real-time PCR using Ct method $(n=7)(B)$ Western blot analysis of Notch1ICD, followed by (C) its densitometric analysis. GAPDH was taken as the loading control. ${ }^{*} p<0.05,{ }^{* *} p<0.01$, $* * * p<0.001$ Vs donors.

\subsection{3 mRNA and protein expression of Notch1 in pulmonary arteries}

To further investigate whether the upregulation of Notch1 observed in lung homogenates from IPAH patients is representative of pulmonary vasculature, Notch1 expression was checked in pulmonary arteries isolated from IPAH and Donor lungs by qRT PCR and western blot. There was no significant change observed in mRNA expression of Notch1 between donor and IPAH (Figure 4.10A). However, at protein level, we observed a substantial increase in Notch1ICD expression in IPAH pulmonary arteries as compared to donors (Figure 4.10B-C).

A)

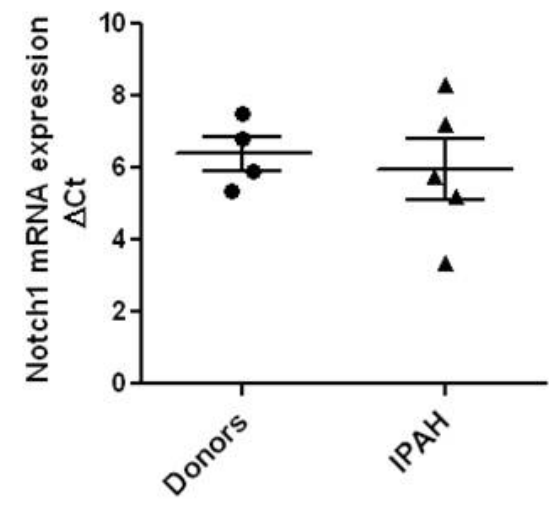

B)

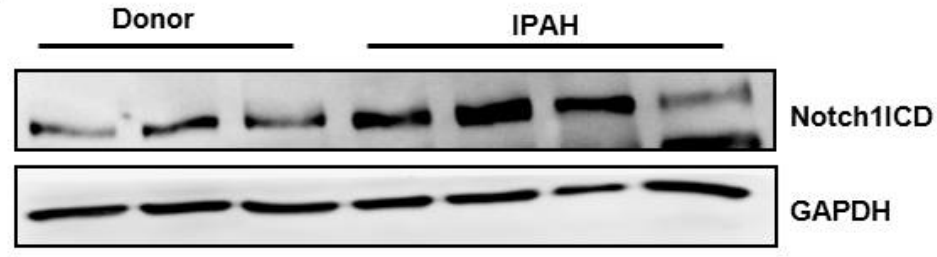

C)

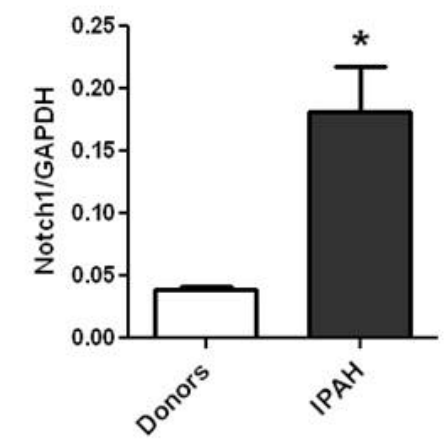

Figure 4.9 Expression of Notch receptors and ligands in Human donors and IPAH pulmonary arteries. (A) mRNA expression of Notch1 in pulmonary arteries of donors and IPAH patients. Regulation at mRNA level 
was analyzed by real-time PCR using Ct method ( $n=3-4)$ (B) Western blot analysis of Notch1ICD, followed by (C) its densitometric analysis. GAPDH was taken as the loading control. ${ }^{*} p<0.05$ Vs donors.

\subsection{Localization of Notch1 in IPAH and donor lungs}

In order to determine the localization of Notch1 in pulmonary vessels, immunostaining of Notch1 was carried out in donor and IPAH human lungs. Only a weak expression of Notch1 was detected in pulmonary arteries of donor lungs (Figure 4.10A-F). In contrast, a strong immunoreactivity of Notch1 was observed in pulmonary arteries of IPAH lung tissues. The expression was confined to the intimal layer, as revealed by serial section staining with vonwillebrand factor (vWF), an endothelial marker and alpha smooth muscle actin ( $\alpha$-actin), a smooth muscle marker (Figure 4.10G-R). 

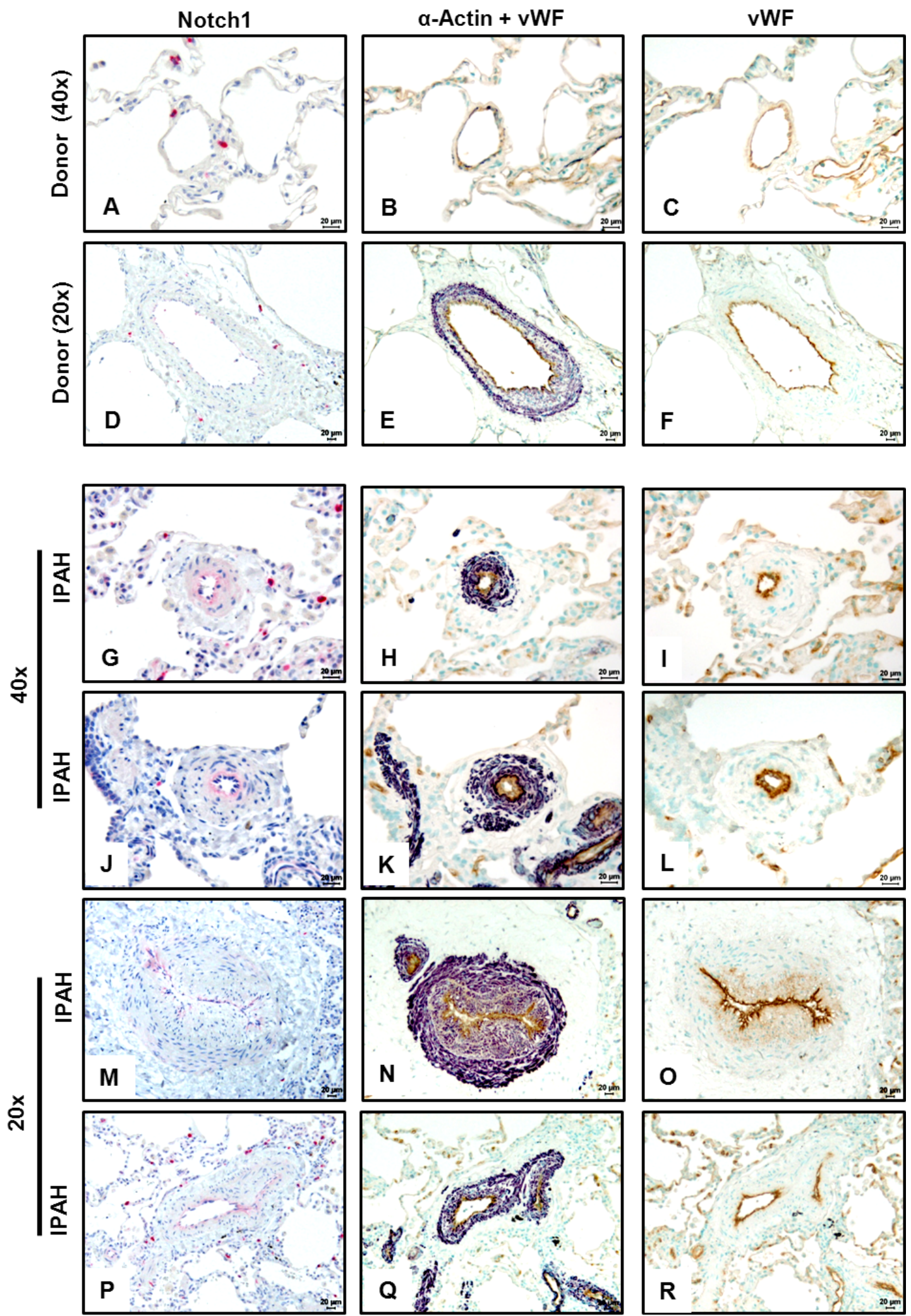

Figure 4.10 Immuno-localization of Notch1 in lungs from IPAH patients and donors. Representative immunostaining microphotographs of the human lung sections from donors (A-F) and IPAH patients (G-R). Staining was undertaken for Notch1, von Willebrand-factor (brown; endothelial cells) and alpha smooth muscle actin (purple; smooth muscle cells). Scale bar: $20 \mu \mathrm{m}$.

\subsection{Effect of Notch1 knockdown on cellular proliferation and apoptosis in hPAECs}


In vitro studies were performed in hPAECs using Notch1 siRNA to investigate the functional role of Notch1 upregulation observed in the initial screening studies.

\subsubsection{Transient Notch1 knockdown by siRNA}

Effective knock down of Notch1 was achieved by using 20nM siRNA in hPAECs. Cells transfected with scrambled siRNA did not show any change in Notch1 expression. Densitometric analysis of the western blot for Notch1ICD showed 85\% knockdown efficiency (Figure 4.11A-B), 48hrs after siRNA transfection. Further, decrease in expression of Hes1, Notch target gene at mRNA level by Notch1 siRNA confirmed functional knock down (Figure 4.11C). In addition, expression of other Notch receptors (Notch2 and Notch4) was analyzed to examine specificity of knockdown. Western blot analysis revealed no effect of Notch1 siRNA transfection on Notch2ICD and Notch4ICD expression (Figure 4.11D).

A)

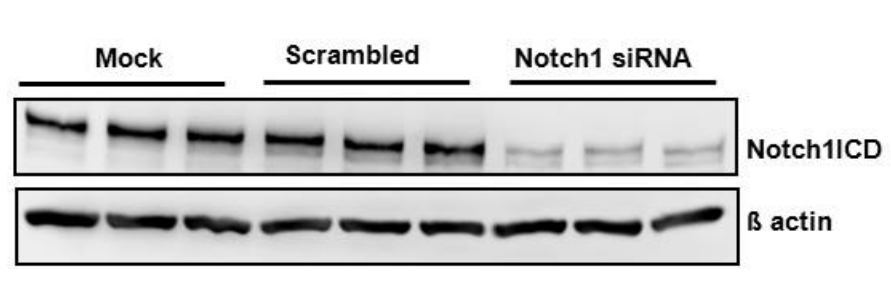

B)

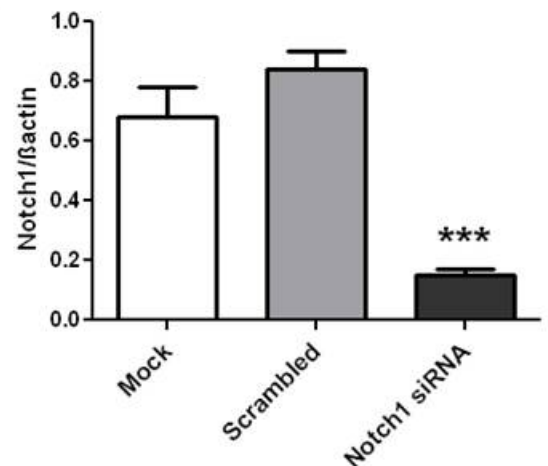

C)

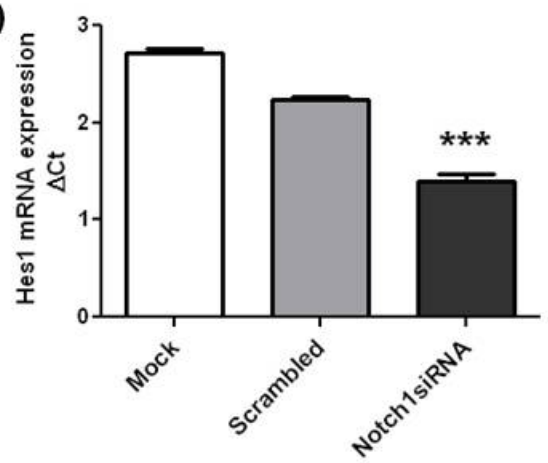

D)

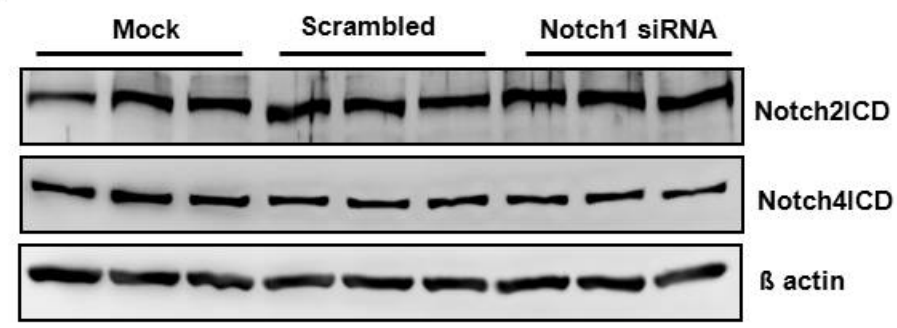

Figure 4.11 Knockdown of Notch1 in hPAECs. hPAECs were transiently transfected with $20 \mathrm{nM}$ of siscramble or si-Notch1 for $48 \mathrm{hrs}$. hPAECs treated with transfection reagent alone is the Mock. Protein expression of Notch1ICD 48hrs after si-Notch1 transfection, as shown by (A) western blot and (B) densitometric analysis of the blot. (C) mRNA expression of Hes1 as measured by real time PCR, 48hrs after si-Notch1 transfection. All values are expressed as $\triangle \mathrm{Ct}$. (D) Notch2ICD and Notch4ICD protein levels $48 \mathrm{hrs}$ after si-Notch1 transfection. Bactin was taken as the loading control. $* * *<0.001$ Vs Mock. $n=3$ in each group. 


\subsubsection{Notch1 knockdown attenuates hPAECs proliferation}

With standardized siRNA knockdown conditions, proliferation of hPAECs was examined 48hrs after Notch1 siRNA transfection using BrdU assay. Importantly, Notch1 knock down decreased ( $40 \%)$ growth medium induced proliferation as compared to scrambled siRNA transfected hPAECs (Figure 4.12A).

A)

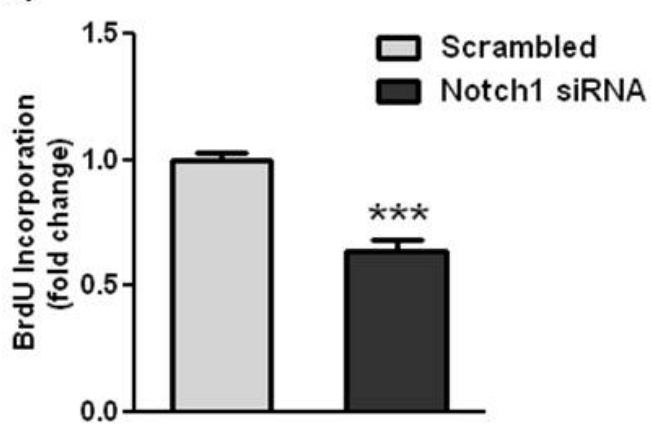

B)

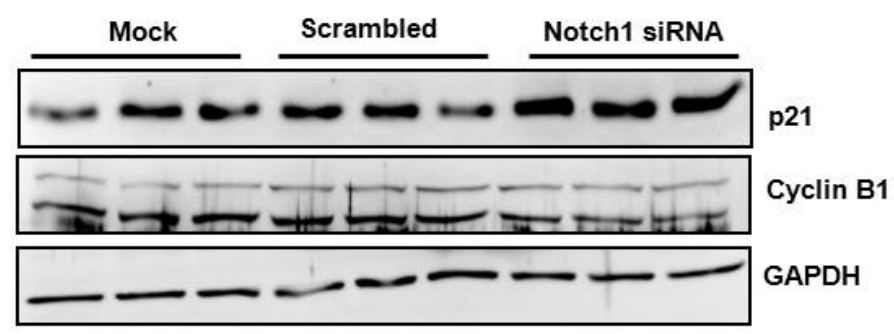

C)
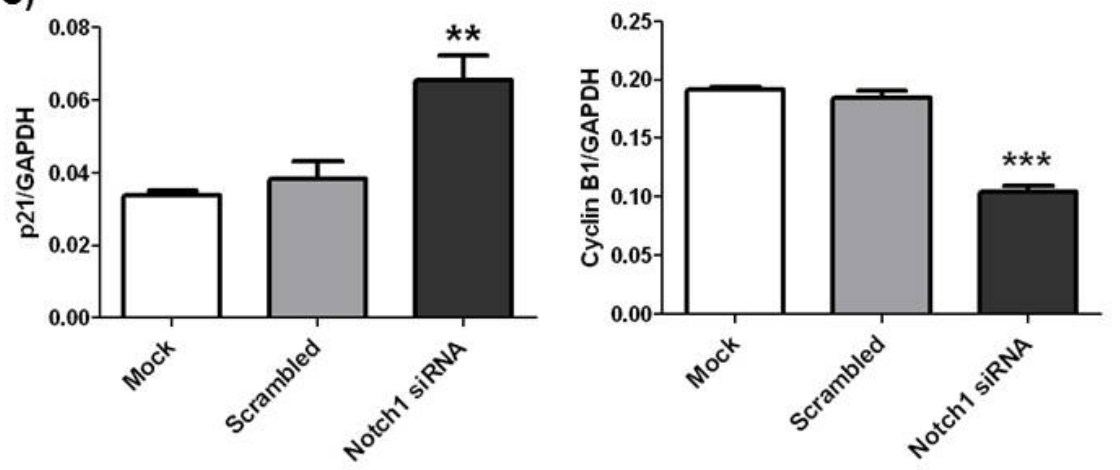

Figure 4.12 Notch1 knockdown attenuates proliferation in hPAECs. (A) hPAECs proliferation as measured by BrdU incorporation, 48hrs after si-Notch1 transfection. ${ }^{* * *} p<0.001$ Vs si-scramble, $(n=5)$. (B) Effect of si-Notch1 on cell cycle regulators was analyzed by western blotting with antibodies against p21 and cyclin B1, followed by (C) densitometric analysis of the blots. GAPDH was taken as the loading control. ${ }^{*} p<0.05$, $* * * p<0.001$ Vs Mock. $\mathrm{n}=3$ in each group.

\subsubsection{Inhibition of Notch1 modulates cell cycle markers}

Cyclins such as cyclinD1, cyclinB1 and cyclin dependent kinase inhibitors (CDKIs) like p21, p27 are very important regulators of cell cycle and hence, proliferation. Therefore, we attempted to study whether any of these cell cycle regulators are modulated by Notch1 knockdown. Western blots, followed by quantification indicated an upregulation of p21 and downregulation of cyclinB1 after si-Notch1 treatment as compared to scrambled siRNA (Figure 4.12B-C). 


\subsubsection{Notch1 knockdown promotes apoptosis in hPAECs}

To determine whether Notch1 plays a role in apoptosis of endothelial cells, TUNEL assay was performed in hPAECs, 48hrs after si-Notch1 transfection. si-Notch1 transfected cells were significantly more sensitive to spontaneous apoptosis as knock down of Notch1 led to 20\% increase in number of apoptotic cells as compared to scrambled siRNA transfected cells (Figure4.13A-B). Additionally, western blots and densitometric quantification for $\mathrm{Bcl}-2$ and $\mathrm{Bax}$ proteins demonstrated a significant decrease in $\mathrm{Bcl}-2$ protein levels in si-Notch1 transfected cells (Figure 4.13C). This resulted in increase of Bax/Bcl-2 ratio (Figure 4.13D), indicative of increased apoptosis.

A)
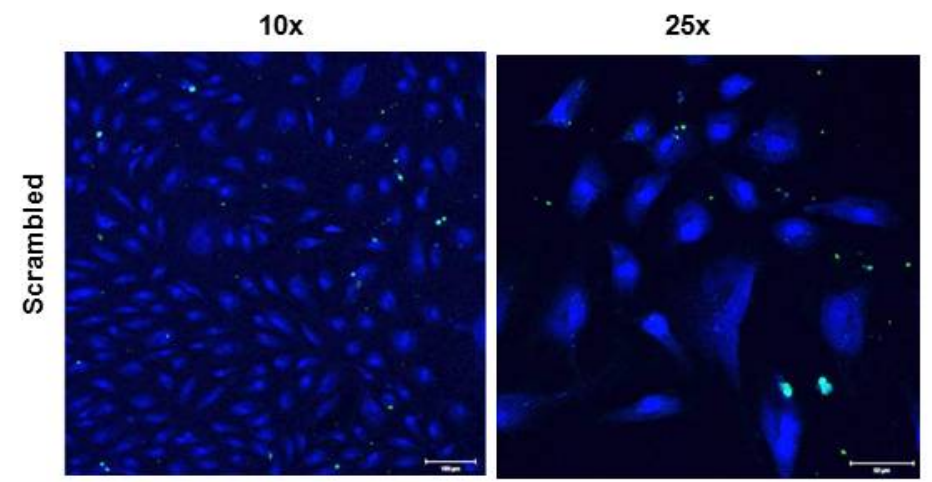

B)
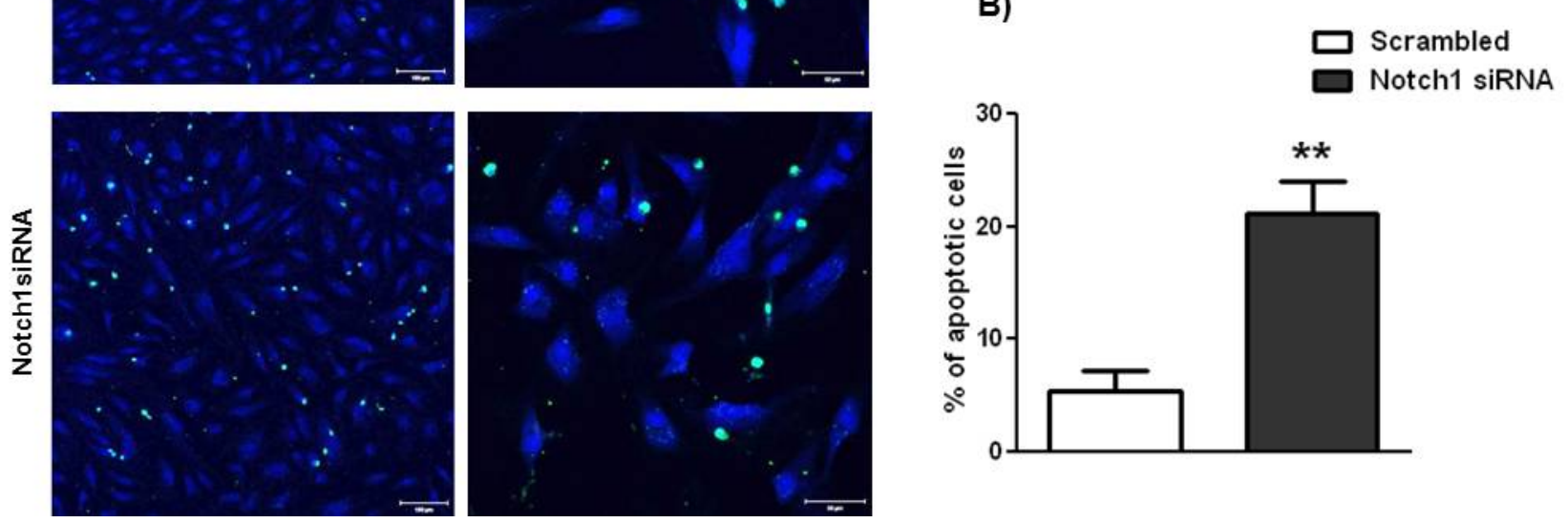
C)

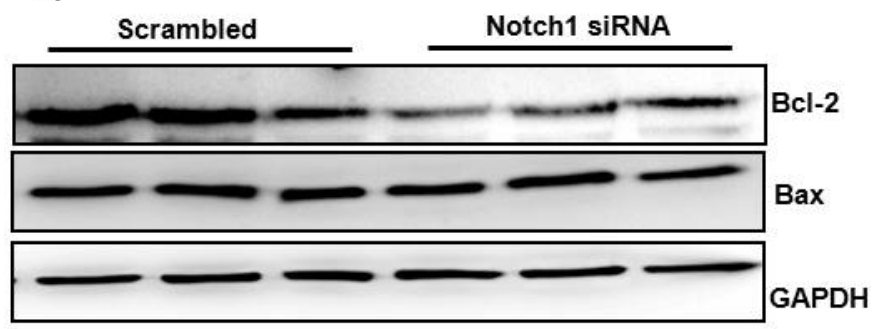

D)

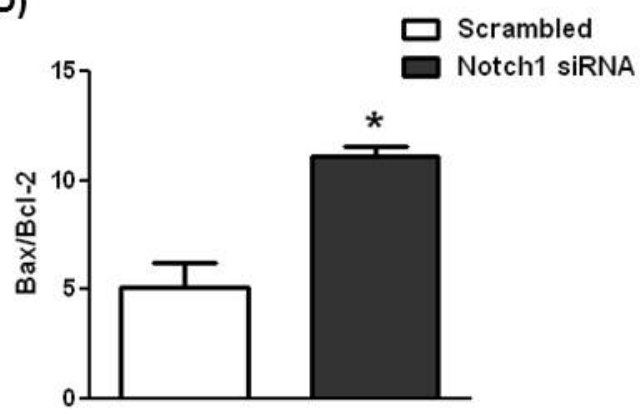

Figure 4.13 Notch1 knockdown induces apoptosis in hPAECs. (A) Representative images of scrambled and si-Notch1 transfected cells that were assessed for apoptosis using in situ cell death detection method (TUNEL assay). (B) Percentage of apoptotic (TUNEL positive) cells as counted using STEPanizer software (C) Effect of Notch1 knockdown was analyzed on apoptotic proteins by Western blotting with antibodies against Bcl-2 and Bax followed by (D) densitometric analysis of the blots. GAPDH was taken as the loading control. ${ }^{*} p<0.05,{ }^{* *} p<0.01$ Vs si-scramble. $n=3$ in each group.

\subsection{Effect of Notch1ICD over expression on cellular proliferation and apoptosis in human pulmonary artery endothelial cells (hPAECs)}

\subsubsection{Overexpression of Notch1ICD in hPAECs}

Transfection with a plasmid containing human Notch1ICD cDNA under CMV promoter (1 $1 \mu \mathrm{g}$ plasmid/well) was carried out to overexpress NotchICD in hPAECs. Same plasmid backbone with GFP under CMV promoter was used as a control plasmid. Western blot analysis using antibody against C-terminal of Notch1 showed that cleaved Notch1 band was strongly up regulated in Notch1ICD transfected cells as compared to control vector transfected cells (Figure 4.14A), 48hrs after transfection. Densitometric analysis confirmed an increase in N1ICD expression by two fold in hPAECs (Figure 4.14B). Transfection efficiency was monitored by observing the cells under microscope for GFP expression in control GFP plasmid transfected cells (Figure 4.14C). Functional overexpression of Notch1 was confirmed by significant increase in mRNA levels of downstream target gene, Hes1 (Figure 4.14D). 
A)

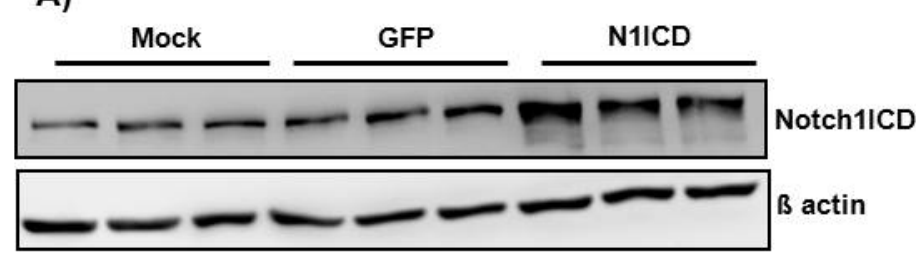

C)
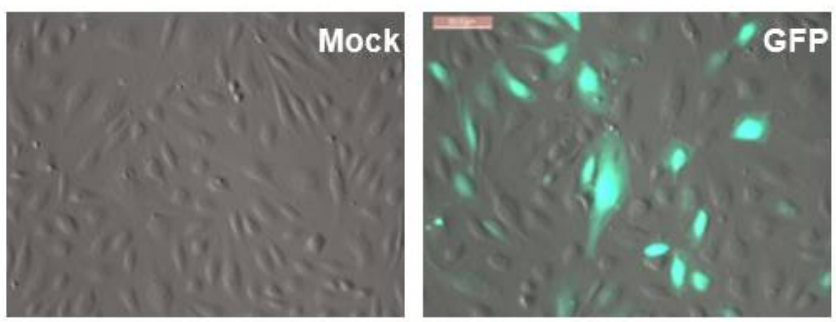

B)

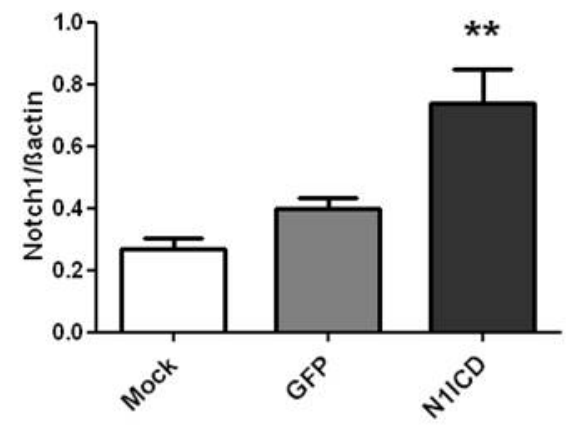

D)

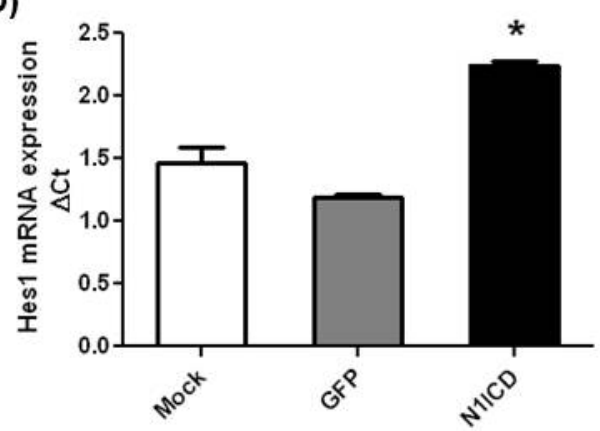

Figure 4.14 Over-expression of Notch1ICD in hPAECs. hPAECs were transiently transfected with $1 \mu \mathrm{g}$ (well/6-well plate) of empty vector or N1ICD plasmid for 48hrs. hPAECs treated with transfection reagent alone is the Mock. (A) Notch1ICD expression, 48hrs after plasmid transfection, as shown by the western blot and (B) densitometric analysis of the same. Bactin was taken as the loading control. (C) Representative images of mock and GFP transfected cells depicting the transfection efficiency. (D) mRNA expression of Hes1 as measured by real time PCR, $48 \mathrm{hrs}$ after si-Notch1 transfection. All values are expressed as $\Delta C \mathrm{Ct} .{ }^{*} \mathrm{p}$ $<0.05$ Vs Mock. $n=3$ in each group.

\subsubsection{Notch1 ICD overexpression increases proliferation of hPAECs}

Proliferation of hPAECs was examined $48 \mathrm{hr}$ after transfection of Notch1ICD and control plasmids, using BrdU incorporation assay. Growth medium stimulated proliferation of hPAECs was increased markedly by $77 \%$ as compared to control plasmid transfected cells (Figure 4.15A).

\subsubsection{Notch1 ICD over-expression modulates cell cycle markers}

In order to check if Notch1 constitutive activity modulated cell cycle regulators, similar to its knockdown, we carried out western blots to check the expression of cell cycle markers, $48 \mathrm{hrs}$ after transfection. Densitometric analysis of the immunoblots demonstrated a significant downregulation in p21 expression while CyclinB1 showed a slight increase in Notch1ICD transfected cells (Figure 4.15B-C). 
A)

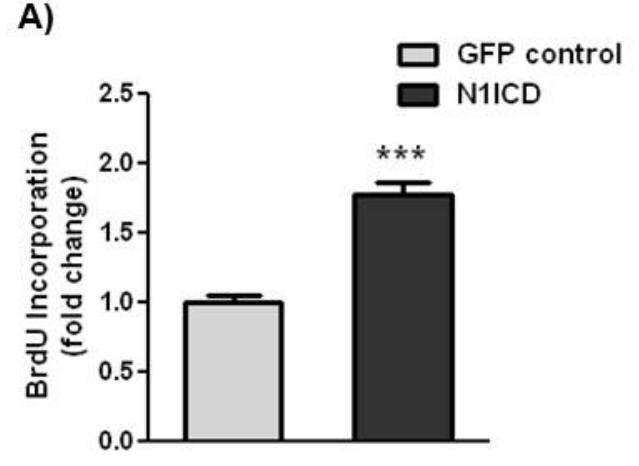

B)

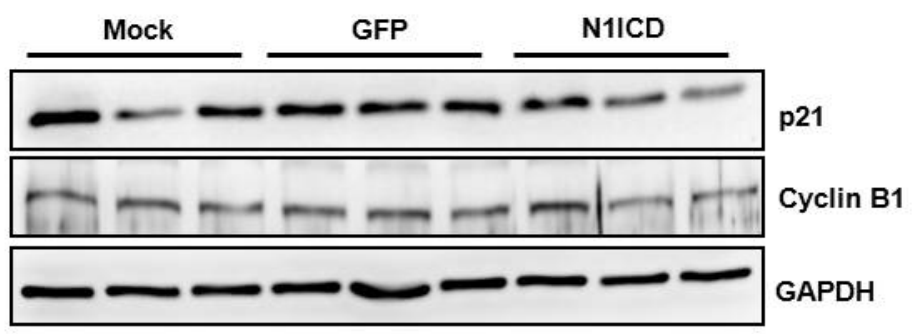

C)
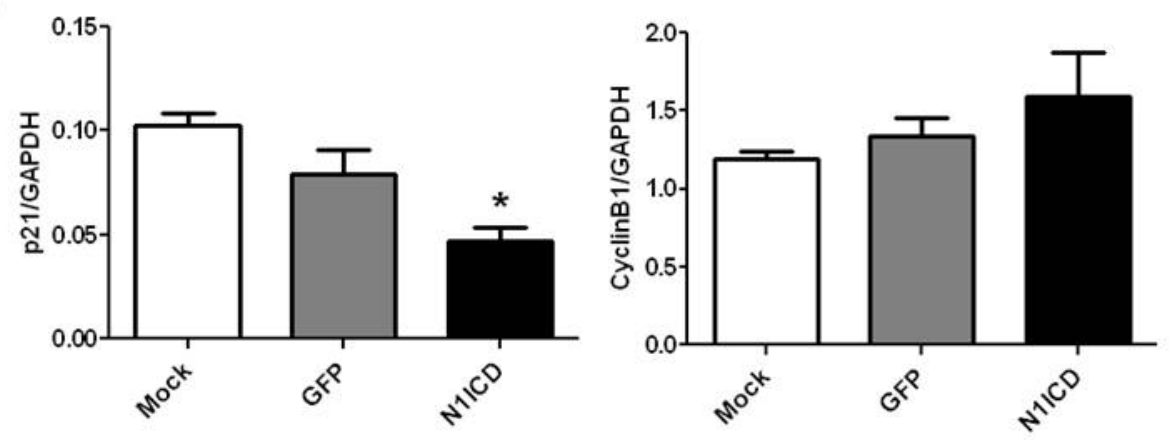

Figure 4.15 Notch1ICD overexpression increases hPAECs proliferation. (A) hPAECs proliferation as measured by BrdU incorporation, 48hrs after N1ICD plasmid transfection. ${ }^{* * *} \mathrm{p}<0.001$ Vs empty vector, $\mathrm{n}=$ 5. (B) Effect of N1ICD overexpression was analyzed on cell cycle regulators by Western blotting with antibodies to p21 and cyclin B1 followed by (C) densitometric analysis of the blots. GAPDH was taken as the loading control. ${ }^{* *} \mathrm{p}<0.05$ Vs Mock. $\mathrm{n}=3$ in each group.

\subsubsection{Notch1 mediates anti-apoptotic effect on hPAECs}

Treatment of hPAECs with Notch1ICD expression plasmid for 48hrs, led to an increase in expression of anti-apoptotic protein, $\mathrm{Bcl}-2$ and a concomitant decrease in pro-apoptotic, Bax protein as observed by western blotting (Figure 4.16A). This led to a significant decrease in $\mathrm{Bax} / \mathrm{Bcl}-2$ ratio (Figure $4.16 \mathrm{~B}$ ) in Notch1ICD transfected cells as compared to empty vector, calculated by quantification of the blots. 
A)

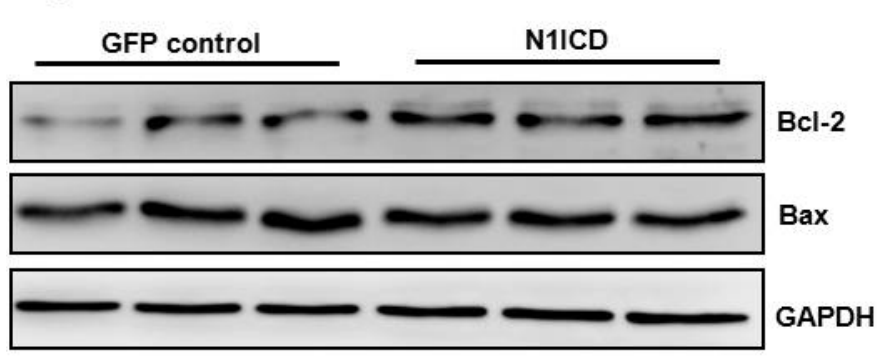

B)

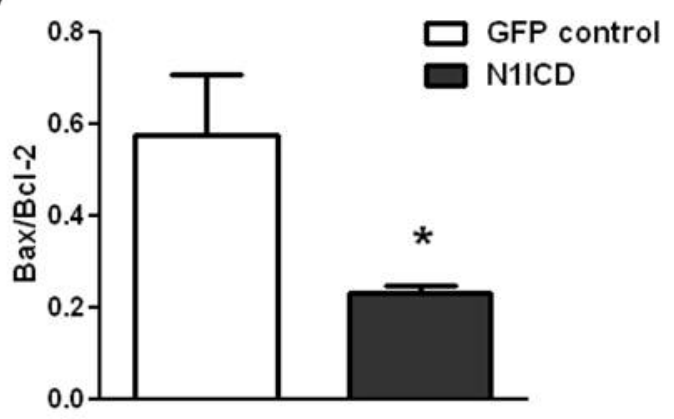

Figure 4.16 Notch1ICD overexpression promotes survival of hPAECs. (A) Effect of N1ICD overexpression was analyzed on apoptotic proteins by Western blotting with antibodies against Bcl-2 and Bax followed by (B) densitometric analysis of the blots. GAPDH was taken as the loading control. ${ }^{*} p<0.05$ Vs empty vector. $\mathrm{n}=3$ in each group.

4.7 Effect of Notch1 knock down and over-expression on proliferation of human pulmonary artery smooth muscle cells (hPASMCs)

In addition to endothelial cells, Notch1 is reportedly expressed in vascular smooth muscle cells. Hence, functional role of Notch1 in proliferation of hPASMCs was also assessed.

\subsubsection{Notch1 knock down has no effect on proliferation of hPASMCs}

$20 \mathrm{nM}$ of siRNA was sufficient to reduce the expression of Notch1 at protein level by $50 \%$ as demonstrated in the western blot for Notch1ICD and its subsequent quantification (Figure 4.17AB). Western blot analysis for Notch3ICD demonstrated that Notch1 siRNA is specific, as Notch1 siRNA transfection did not have any significant effect on expression of Notch3 (Figure 4.17C). Proliferation of hPASMCs was examined $48 \mathrm{hr}$ after si-Notch1 transfection using BrdU incorporation assay. Interestingly, there was no change observed in growth medium stimulated proliferation of hPASMCs after knockdown of Notch1 (Figure 4.17D). 

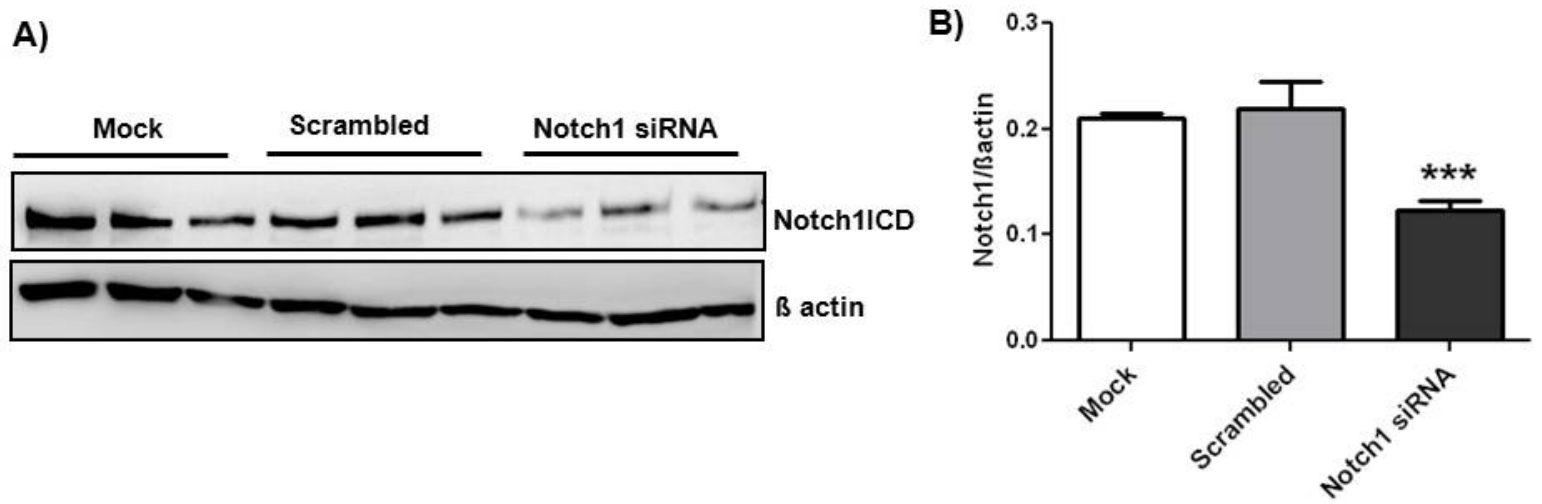

C)
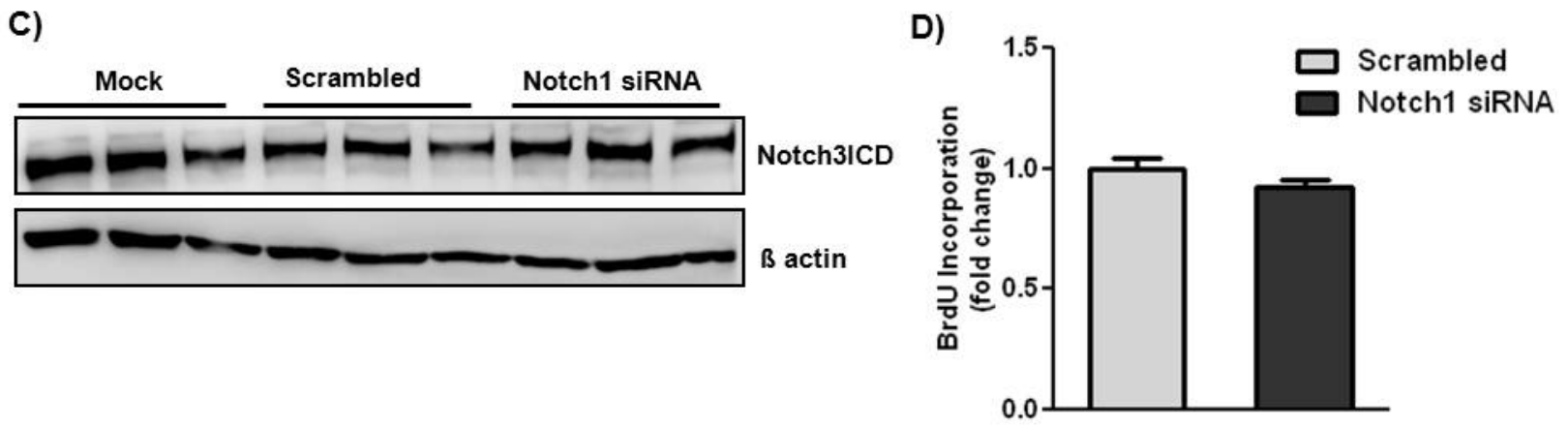

Figure 4.17 Knockdown of Notch1 in hPASMCs. hPASMCs were transiently transfected with 20nM of siscramble or si-Notch1 for 48hrs. hPASMCs treated with transfection reagent alone is the Mock. Notch1ICD expression, 48hrs after si-Notch1 transfection, as shown by (A) western blot and (B) densitometric analysis of the same. (C) Notch3ICD protein levels 48hrs after si-Notch1 transfection. $B$ actin was taken as the loading control. ***p $<0.001$ Vs Mock. $\mathrm{n}=3$ in each group. (D) hPASMCs proliferation as measured by BrdU incorporation, 48hrs after si-Notch1 transfection.

\subsubsection{Notch1 over-expression has no effect on proliferation of hPASMCs}

Transfection with a plasmid containing Notch1ICD cDNA under control of CMV promoter was carried out to obtain constitutive expression of Notch1ICD in hPASMCs. Transfection resulted in a $\sim 4$ fold increase in expression of Notch1ICD as demonstrated in western blot against Notch1ICD (Figure 4.18A-B). Constitutive expression of Notch1ICD did not have any effect on growth medium stimulated proliferation of hPASMCs as measured by BrdU incorporation assay (Figure 4.18C). 
A)

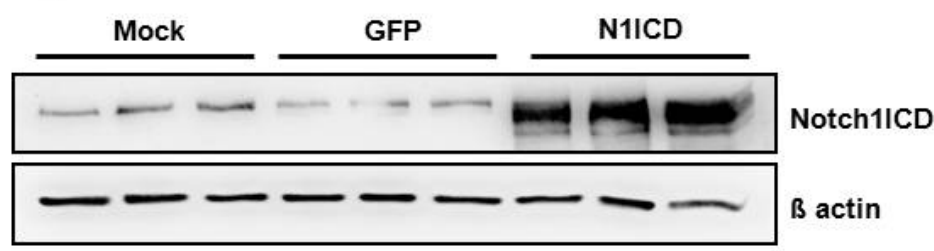

B)

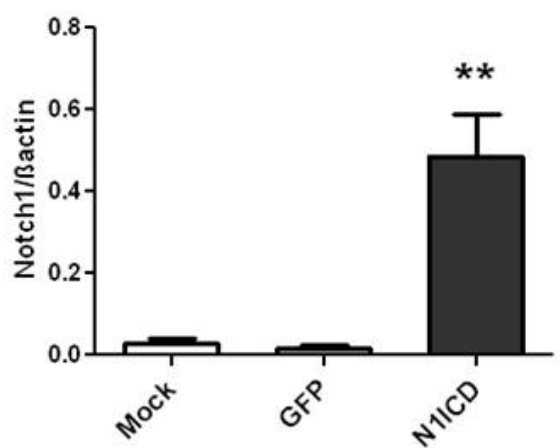

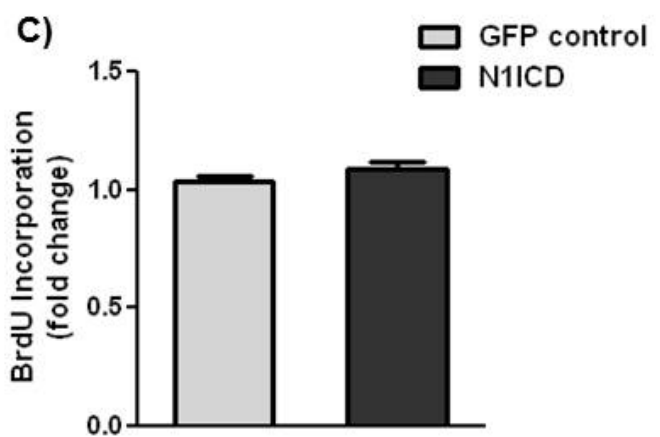

Figure 4.18 Overexpression of Notch1ICD in hPASMCs. hPASMCs were transiently transfected with $1.5 \mu \mathrm{g}$ (per well of 6-well plate) of empty vector or N1ICD plasmid for 48hrs. PASMCs treated with only transfection reagent is the Mock. (A) Notch1ICD expression, $48 \mathrm{hrs}$ after plasmid transfection, as shown by the western blot and (B) densitometric analysis of the same. Bactin was taken as loading control. ${ }^{* *} p<0.01$ Vs Mock. $n=3$ in each group. (C) hPASMCs proliferation as measured by BrdU incorporation, 48hrs after N1ICD plasmid transfection.

\subsection{Effect of pharmacological inhibition of Notch signaling using $\gamma$-secretase inhibitor, DBZ on hPAECs and hPASMCs}

\subsubsection{Inhibition of Notch signaling by $\gamma$-secretase inhibitor, DBZ}

To study the functional role of Notch signaling in hPAECs and hPASMCs, $y$-secretase inhibitor, DBZ was used to inhibit the endogenous notch activity. $\gamma$-secretase inhibitor binds and inhibits the enzyme complex, $\gamma$-secretase, responsible for cleavage of Notch receptors. Treatment with DBZ decreased $\mathrm{\gamma}$-secretase activity by $50 \%$ after $8 \mathrm{hrs}$ in hPAECs as observed by the reduced protein levels of Notch1ICD (Figure 4.19A-B). Furthermore, DBZ dose dependently decreased the expression of Hes1, downstream target gene of notch signaling pathway in both hPAECs (Figure 4.19C) and hPASMCs (Figure 4.19D). 
A)

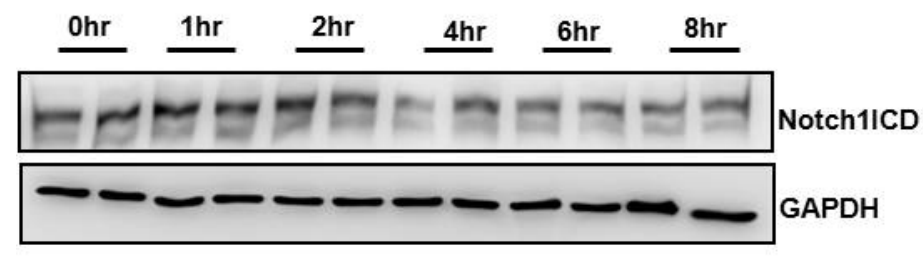

C)

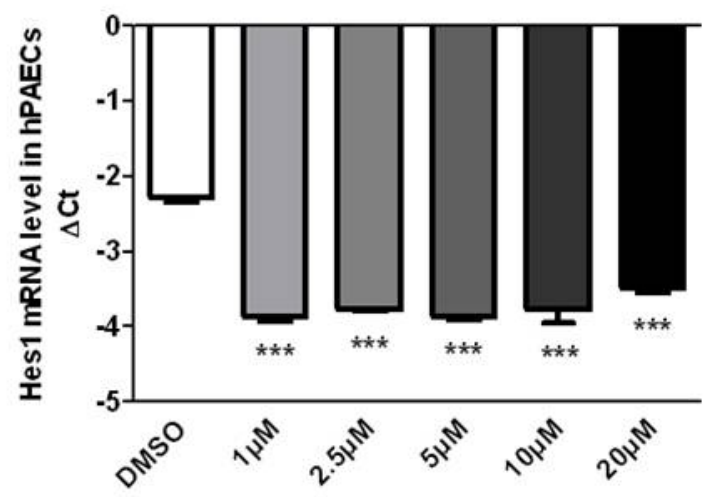

B)

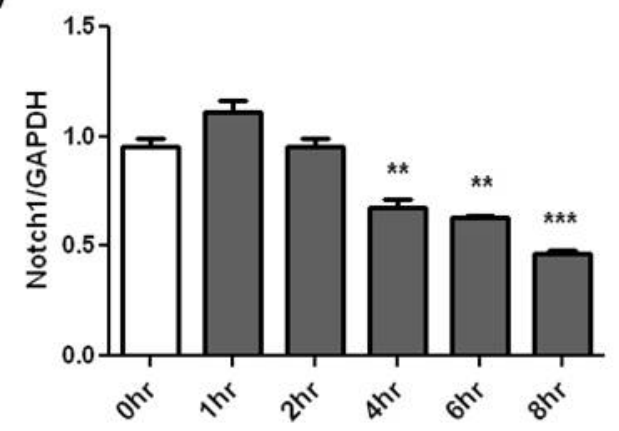

D)

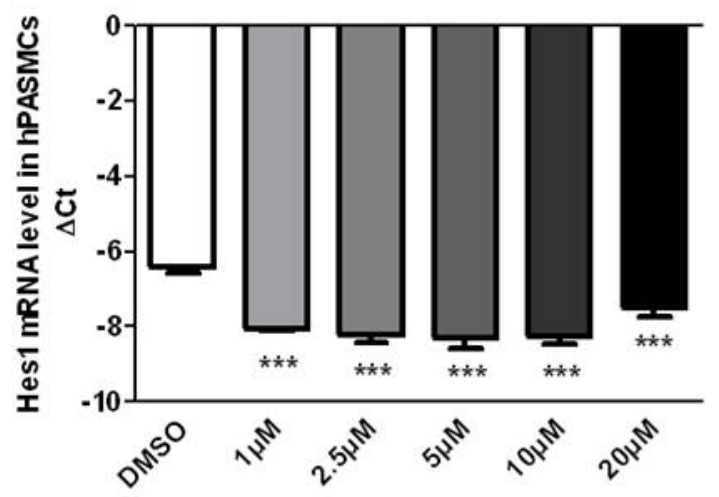

Figure $4.19 \gamma$ secretase inhibitor (DBZ) inhibits Notch signaling in hPASMCs and hPAECs. (A) Western blot analysis of Notch1ICD after treatment of hPAECs with DBZ with GAPDH as the loading control. (B) Quantification of the Western Blot. GAPDH was taken as the loading control. ${ }^{* *} \mathrm{p}<0.01,{ }^{* * *} \mathrm{p}<0.001 \mathrm{Vs}$. hr. mRNA expression of Hes1 in (C) hPAECs and (D) hPASMCs after 24 hrs treatment with DBZ as analyzed by real time PCR. All values are expressed as $\Delta$ Ct. ${ }^{* * *} p<0.001$ Vs DMSO control.

\subsubsection{DBZ attenuates proliferation of hPAECs}

We examined the effect of DBZ on proliferation of hPAECs by stimulating quiescent hPAECs with $5 \%$ FCS or VEGF $(10 \mathrm{ng} / \mathrm{ml})$ in presence/absence of DBZ using BrdU incorporation assay. Treatment with 5\% FCS resulted in a marked increase in proliferation of hPAECs (by $88 \%$ ) which was reduced to less than half (by $66 \%$ ) by $20 \mu \mathrm{M}$ DBZ (Figure $4.20 \mathrm{~A}$ ). Interestingly, proliferation induced by VEGF, a potent mitogen for hPAECs, was also reduced effectively by $20 \mu \mathrm{M}$ DBZ by $56 \%$ (Figure 4.20B). 
A)

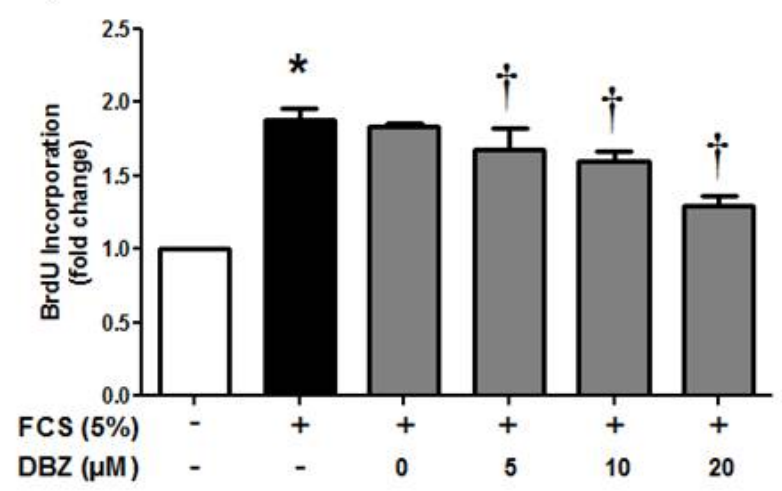

B)

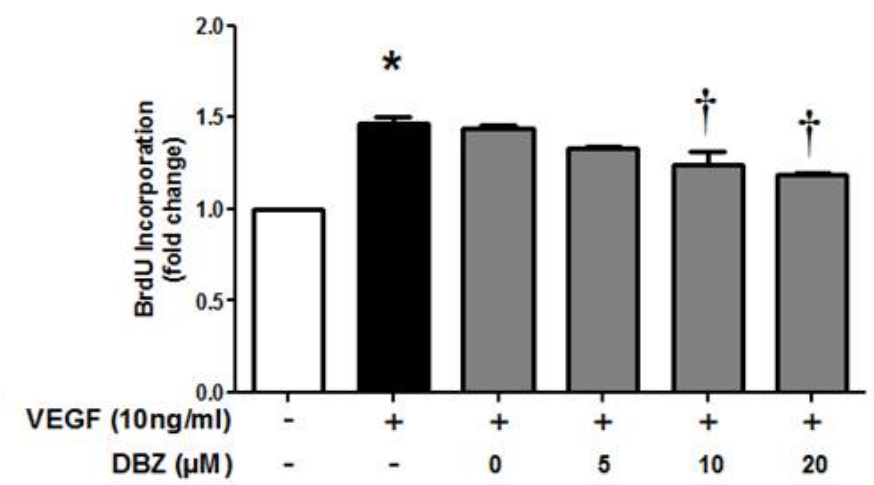

Figure 4.20 v secretase inhibitor (DBZ) attenuates proliferation in hPAECs. hPAECs were serum starved and stimulated with (A) $5 \%$ FCS or (B) $10 \mathrm{ng} / \mathrm{ml}$ VEGF in presence of various concentrations of DBZ or DMSO $(-)$ and measured for the changes in proliferation by BrdU incorporation. All data are expressed as fold increase of the non-stimulated group and represent means $\pm S E M, n=5 .{ }^{*}, p<0.05$ Vs non-stimulated; ${ }^{\dagger}$, $p<0.05$ Vs (A) FCS-treated group or (B) VEGF- treated group.

\subsubsection{DBZ inhibits proliferation of hPASMCs}

Effect of DBZ on proliferation of hPASMCs was also investigated using BrdU incorporation assay. Serum starved hPASMCs were stimulated with $5 \%$ FCS or PDGF-BB (10ng/ml) in presence/absence of DBZ. Interestingly, DBZ significantly decreased DNA synthesis in both, 5\% FCS (Figure 4.21A) and PDGF-BB (Figure 4.21B) stimulated hPASMCs in a dose-dependent manner with maximum inhibition observed at $20 \mu \mathrm{M}$ (by $86 \%$ for $5 \%$ FCS and $76 \%$ for PDGF-BB).

A)

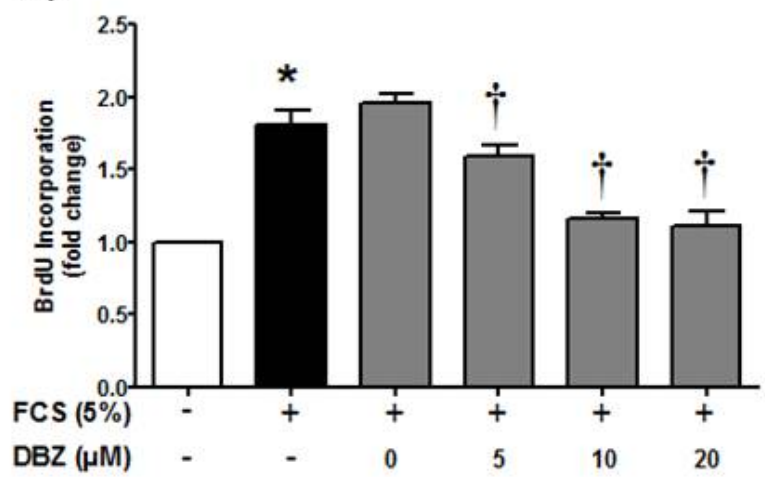

B)

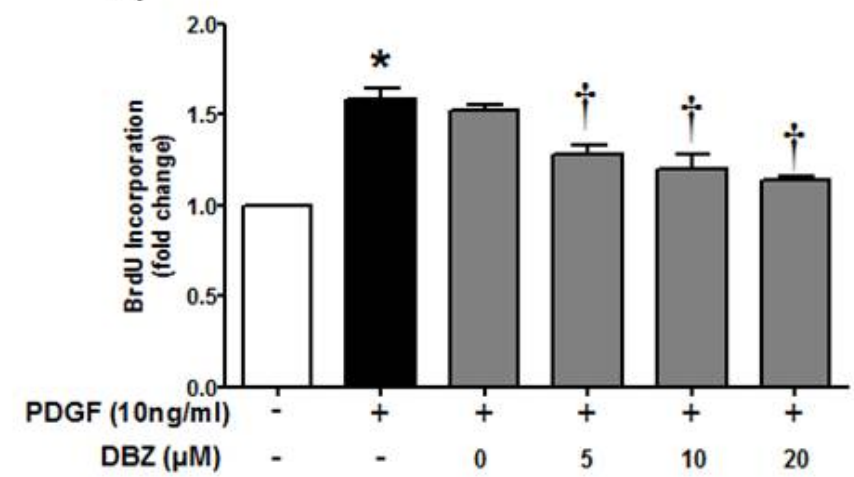

Figure $4.21 \gamma$ secretase inhibitor (DBZ) attenuates proliferation in hPASMCs. hPASMCs were serum starved and stimulated with (A) $5 \%$ FCS or (B) $10 \mathrm{ng} / \mathrm{ml}$ PDGF-BB in the presence of various concentrations of DBZ or DMSO (-) and measured for the changes in proliferation by BrdU incorporation. All data are expressed as fold increase of the non-stimulated group and represent means $\pm S E M, n=5 .{ }^{*}, p<0.05$ Vs nonstimulated; $t, \quad p<0.05$ Vs (A) FCS-treated group or (B) PDGF-BB treated group. 


\section{DISCUSSION}

Research carried out in past decade has very well established Notch signaling as an important player in various aspects of vascular development, homeostasis $[128,157,158]$ as well as vascular injury $[132,137,138]$. Indispensable involvement of Notch3 in arterial smooth muscle cell identity $[124,125]$ and Notch1 and Notch4 in arterial endothelial phenotype and angiogenesis [119], provides intriguing prospects of their involvement in the pathogenesis of pulmonary hypertension. Recently, Li et al have reported increased activation of Notch3 signaling in PASMCs from PAH patients. They further demonstrated Notch3 deletion protected mice against development of hypoxia induced PH [147]. Interestingly, this is the first and only study to date, suggesting a link between Notch signaling and PAH development. Notch receptors, other than Notch3, have also been implicated in vascular homeostasis and neointima formation after vascular injury $[138,139]$. Hence, it is important to study the expression pattern of the other Notch receptors and ligands and to further determine their role in pathophysiology of $\mathrm{PH}$.

\subsection{Expression of Notch receptors and ligands in IPAH patients}

A complete expression analysis at mRNA and protein levels was carried out in lungs of IPAH patients and donors. Profound changes were detected in Notch signaling molecules in lung homogenates of IPAH patients as compared to donors. Notch receptors (Notch1, 3 and 4) and ligands (Jagged1, DLL1) were significantly upregulated in IPAH lungs at the mRNA level (Figure 4.9A). Although, western blot analysis did not indicate significant differences in Notch3ICD, Notch4ICD, Jagged1 and DLL1 at protein levels (data not shown). This suggests a discrepancy between transcription and translation regulation of these mentioned proteins and should be investigated in future. Interestingly, a major increase was observed in Notch1ICD protein levels in IPAH lungs indicating an increased activation of Notch1 signaling pathway in lungs of IPAH patients as compared to donors (Figure 4.9B-C).

To further validate, that the changes observed in Notch1 were localized to pulmonary vasculature, Notch1 expression was analyzed in pulmonary arteries isolated from IPAH patients and donors. Indeed, increased protein expression of Notch1ICD was detected in arteries from IPAH patients. Though, there was no significant change observed in the mRNA expression (Figure 4.10). To our knowledge, this is the first study demonstrating an increased Notch1 activation in pulmonary vasculature of IPAH patients. 


\subsection{Expression of Notch receptors and ligands in experimental PH models}

In present investigation, established animal models of $\mathrm{PH}$ were employed to further study the role of Notch signaling in pathogenesis of $\mathrm{PH}$. To begin with, expression studies at mRNA and protein levels were performed in MCT induced PH rats. These studies demonstrated altered expression of Notch receptors and ligands in lungs of MCT induced PH rats. At the mRNA level, Notch3 exhibited a strong upregulation while Notch1, Notch4 and DLL4 were significantly decreased (Figure 4.2). MCT induced PH model is characterized by medial hypertrophy and the underlying mechanism of this model is thought to be MCT pyrole mediated endothelial damage, triggering release of growth factors and cytokines [149]. Notch4 and DLL4 are reported to be exclusively expressed in endothelial cells [158] and furthermore, Notch1 is abundantly expressed in endothelium [159], hence, their observed decrease in expression might be associated with endothelial damage. In accordance, a reduced protein expression of Notch1ICD, Notch4ICD and DLL4 was detected in MCT lungs. However, Notch3ICD western blot analysis revealed a decrease in MCT lungs as compared to the healthy rat lungs, contrary to its mRNA expression (Figure 4.3). Though not extensively investigated, Notch3 expression is also observed in macrophages [160] and interestingly, MCT model of $\mathrm{PH}$ is characterized by adventitial inflammation, particularly macrophage accumulation [20,150]. Hence, increase in Notch3 mRNA could represent the basal expression of infiltrating inflammatory cells, along with smooth muscle cells and not the activated Notch3 signaling (as no increase was detected at the level of Notch3ICD in protein blots). It is well established that notch receptor activation and cleavage is dependent on ligand availability and binding. Therefore, a decrease observed in Notch3ICD protein levels could be a direct outcome of the strong decrease observed in the protein levels of ligands (DLL4, DLL1 and Jagged1) in MCT lung homogenates.

The MCT model is recognized as the classical experimental $\mathrm{PH}$ model to study pathophysiological mechanisms and therapeutic options for the disease. However, it only represents the pathological features of group 1 (PAH) of the Dana Point classification of PH (Table 1). In order to analyze the contribution of notch signaling in $\mathrm{PH}$ due to different etiology, we further investigated expression of notch receptors and ligands in Chronic Hypoxia induced PH mice model. Chronic hypoxia model represents group 3 ( $\mathrm{PH}$ owing to lung diseases and/or hypoxia) of the Dana Point classification [151] and furthermore, addresses the aspect of interspecies differences. Screening studies carried out in hypoxia mice model indicated a substantial increase in 
Notch3 and downregulation in DLL4 expression at mRNA level in hypoxic mice lungs (Figure 4.5A). In agreement with these results, upregulation in Notch3ICD and DLL4 was also observed at the level of protein expression in hypoxic mice lungs (Figure 4.5B). Chronic Hypoxia exposure of mice is characterized by minimal vascular remodeling, depicted mainly by medial hypertrophy and no or negligible endothelial damage or neointima formation [148, 151]. Hence, the significant decrease observed in MCT model for some Notch receptors (Notch4 and Notch1) are not seen here. Interestingly, Notch pathway is known to intersect with hypoxic signaling. Using oxygen-induced retinopathy mice model, Liu et. al. have shown that Notch3 is induced in retinal vasculature upon hypoxic stimulation. On induction, it cooperates with HIF-1 $1 \alpha$ to regulate angiopoietin-2 expression contributing to neovascularization [161]. Additionally, Notch3 has been recently demonstrated to be involved in pathogenesis of chronic hypoxia induced PH in mice [147]. Our results exhibiting an upregulation of Notch3 expression and cleavage in the lungs of hypoxia exposed mice are in line with these findings.

MCT injection in rats and Hypoxia exposure of mice are the commonly employed models of $\mathrm{PH}$, mimicking medial hypertrophy observed in the disease condition [148, 151].Additional pathological changes observed in human pulmonary arterial hypertension, namely; intimal proliferation and plexiform lesions are absent in these models. Hence, they may not be able to recapitulate all the changes occurring in the human disease. A relatively new rat model, involving hypoxia exposure after Su5416 administration has been developed [155], capable of emulating formation of neointimal lesions. Expression analysis of Notch family in lung homogenates from Hypoxia+Su5416 rats presented a similar picture as observed in IPAH patients. At the mRNA level, many Notch receptors (Notch1, Notch2, and Notch3) and ligands (Jagged1, Jagged2) were increased while ligand DLL4 was downregulated in the Hox+Su5416 rats (Figure 4.7A). Western blots followed by densitometric quantification revealed an upregulation of only Notch3ICD and Notch1ICD proteins in lungs of Hox+Su5416 rats as compared to healthy rats (Figure 4.7B-C). Unlike MCT and chronic hypoxia exposure, Hox+Su5416 rats are reported to mirror the hyperproliferative endothelial cell etiology i.e. plexiform lesions, a hallmark of severe PAH. Hence, the heightened expression of Notch1 only observed in Hox+Su5416 rat lungs among the animal models, in agreement with the human data, hint towards its role in plexiform lesions. Plexiform lesions, consisting of hyperproliferative altered endothelial cells are generally observed at branching points of pulmonary arteries in patients with IPAH and are believed to be an outcome of 
increased shear stress $[162,163]$. Similarly, VEGFR2 blockade by Su5416 with hypoxia exposure is shown to mirror these shear stress conditions, leading to formation of plexiform lesions in rats [164]. Interestingly, an in vitro study has demonstrated increased activation of Notch1 in ES cellsderived VEGFR2 ${ }^{+}$cells, under shear stress [165], providing impetus to our hypothesis.

\subsection{Localization of Notch1 in IPAH lungs}

Unlike Notch3, exclusively expressed in SMCs, Notch1 exhibits a more widespread expression. Several reports have indicated Notch1 expression in endothelial cells regulating apoptosis and proliferation $[130,166]$. Additionally, Notch1ICD was also found to regulate SMC growth, apoptosis, and migration through CBF-1/RBP-Jk- dependent pathways [139, 167]. Immunohistochemical analysis of Notch1 in human lung tissues from donors and IPAH patients revealed localization of Notch1 in intimal layer of pulmonary arteries. In line with screening results, a stronger expression of Notch1 was observed in intima of IPAH lungs as compared to donor lungs.

Although well characterized in systemic vasculature $[127,158]$ and vascular injury [137, 138], Notch1 is poorly addressed in pulmonary vasculature. The significant increase observed in Notch1 expression and cleavage in lungs and pulmonary arteries of IPAH patients as well as lungs of Hox+Su5416 rats in our initial screening experiments suggest that Notch1 might contribute to pathogenesis of $\mathrm{PH}$. Based on these results, we carried out in vitro studies to analyze the functional role of Notch1 signaling in human pulmonary arterial endothelial cells.

\subsection{Influence of Notch1 on proliferation of hPAECs}

Interestingly, endothelial cells within plexiform lesions of patients with PPH expand in a monoclonal fashion, i.e., they arise from a single cell $[168,169]$. As monoclonal cell growth has been consistently demonstrated in cancer and in smooth muscle cells within atherosclerotic plaques, it was proposed that endothelial cell proliferation in PPH displays mechanistic features common to neoplastic cell growth [170]. Consistent with a neoplastic hypothesis of severe PAH, endothelial cells obtained from the pulmonary arteries of patients with IPAH have been characterized to be hyperproliferative and apoptosis resistant [171]. Notch1 aberrant activation has been indicated in a variety of cancers. Activated Notch1 signaling is shown to contribute to $\sim 50 \%$ of human T-cell acute lymphoblastic leukemia (T-ALL) cases through gain of function mutations in Notch1 gene [172] where it contributes to the hyper proliferative and survival of the 
cancerous cells [173]. Later, Notch signaling was further shown to be associated with various solid tumors, including breast cancer, colorectal cancer, non-small cell lung carcinoma (NSCLC), and melanoma [174]. Similarities between cancerous cells and altered endothelial cells of plexiform lesions and the established role of Notch1 as an oncogene, led us to examine its contribution in endothelial proliferation and apoptosis. Hence, in our studies, we examined the effect of Notch1 knock down and over activity on proliferation of endothelial cells in vitro.

In our studies, Notch1 downregulation, attained by specific siRNA mediated knock down, resulted in a $\sim 40 \%$ decrease in proliferation of hPAECs, measured by BrdU incorporation (Figure 4.12A). On the other hand, Notch1ICD over expression gave rise to a significant $~ 75 \%$ increase in proliferation of hPAECS (Figure 4.15A). Some earlier reports have implicated Notch1 in regulation of endothelial proliferation, however, with conflicting conclusions. In human aortic endothelial cells, Notch1ICD over expression was shown to inhibit proliferation of the cells via repression of MAPK and PI3K/Akt pathway [175]. Another study also revealed induction of endothelial cell cycle arrest by Notch1 and Notch4 activation with the aid of p21 repression [130]. Contradictory to these reports, Takeshita et. al. demonstrated that Notch1 is essential for VEGF induced proliferation, migration and survival of endothelial cells [166]. Using transgenic mice (N1+//ecN1 $1^{+/} / \mathrm{N}^{-/-}$), authors showed that Notch1, but not Notch4 is critical for VEGF induced post ischemic angiogenic response. In accordance with this report, another study linked reduced Notch1 cleavage with decreased proliferating cell nuclear antigen (PCNA) levels in endothelial cells, when cocultured with Jagged1 deficient vSMCs [176].

Our study supports the positive role of Notch1 signaling in proliferation of pulmonary artery endothelial cells. Cell proliferation is tightly regulated by expression and activation of cell cycle regulators, cyclins, cyclin dependent kinases (Cdks) and Cdk inhibitors (Cdkls). Cdk inhibitors like p21 and p27 have negative effects on cell cycle machinery by binding to cyclin-Cdk complexes and inhibiting them [177]. To explore the mechanism involved in Notch1 mediated regulation of cell proliferation, the expression of cell cycle proteins was examined. The cell cycle markers, namely p21 and cyclinB1 were found to be modulated by Notch1 knockdown and overexpression in hPAECs. We observed a marked increase in protein levels of cell cycle inhibitor, p21 and significant decrease in mitogenic protein, cyclinB1 with Notch1 knockdown (Figure 4.12B-C). Notch1ICD over expression was associated with decrease in the expression of p21 as detected by 
immunoblotting (Figure 4.15B-C). Though, cyclinB1 expression did not show any significant change with Notch1 over expression. p21, a well-known Cdkl, has been described as a direct target of RBPJK dependent Notch1 signaling [109, 130], where authors demonstrated repression of p21 promoter activity under Notch1 activation in HUVECs. Our results indicate towards a role of Notch1 in hPAECs cell cycle regulation via direct effect on p21 expression.

\subsection{Effect of Notch1 on survival of hPAECs}

Resistance to apoptosis is another important characteristic of altered endothelial cells found in the plexiform lesions of patients with PAH [171]. DNA isolated from microdissected endothelial cells within plexiform lesions of patients with PPH exhibit mutations within Bax gene, a proapoptotic member of $\mathrm{Bcl}-2$ family of proteins. Additionally, these endothelial cells have reduced expression of Bax protein [170]. Another study demonstrated decreased expression of antiapoptotic protein, $\mathrm{Bcl}-2$ in endothelial cells isolated from plexiform lesions [171]. These findings prompted us to analyze the effect of Notch1 knock down and overexpression on apoptosis of hPAECs. siRNA mediated knockdown of Notch1 displayed a small but significant (20\%) increase in number of TUNEL-positive apoptotic vascular cells compared to scrambled siRNA transfected cells (Figure 4.13A-B). At the same time, Bax/Bcl-2 ratio was utilized to substantiate the findings from TUNEL assay. It is known that Bax and $\mathrm{Bcl}-2$ regulate apoptosis downstream of p53 [178]. Bcl-2 blocks cell death following various stimuli, demonstrating an anti-apoptotic effect; however, overexpression of Bax has a pro-apoptotic effect and Bax also counters the antiapoptotic activity of $\mathrm{Bcl}-2[179,180]$. Many reports proposed and further, demonstrated that the ratio of $\mathrm{Bcl}-2$ to Bax might govern the sensitivity of cells to apoptotic stimuli [181, 182]. Interestingly, we were able to observe a significant decrease in $\mathrm{Bcl}-2$ protein levels and concomitant increase in Bax protein expression on treatment of hPAECs with Notch1 siRNA as compared to scrambled, thus, lowering $\mathrm{Bcl}-2 / \mathrm{Bax}$ ratio, indicative of increased apoptosis (Figure 4.13C-D). In agreement, Notch1ICD constitutive expression led to an increased protein expression of $\mathrm{Bcl}-2$, hence, an increased $\mathrm{Bcl}-2 / \mathrm{Bax}$ ratio (Figure 4.16A-B).

Previous studies have defined an anti-apoptotic role of Notch1 signaling in both animal and in vitro cell models [183], in the field of development and cancer. In endothelial cells, Notch4 has been shown to inhibit apoptosis, in response to inflammatory mediator, lipopolysaccharides (LPS) via RBP-JK-dependent and -independent pathways [129]. However, not much is known about involvement of Notch1 in endothelial apoptosis, in vascular injury. Our results suggest a role of 
Notch1 in promoting survival of hPAECs by regulating expression of $\mathrm{Bcl}-2$ and to some extent, Bax.

\subsection{Effect of Notch1 on proliferation of hPASMCs}

It has been previously demonstrated that activated Notch1 in SMCs mediated neointimal formation after carotid artery ligation by regulating proliferation, apoptosis and migration of these cells [139]. This led us to investigate the involvement of Notch1 in proliferation of hPASMCs in vitro. However, siRNA mediated Notch1 knock out did not show any effect on proliferation of hPASMCs (Figure 4.17D) and neither Notch1 constitutive activation via Notch1ICD overexpression effected proliferation as measured by BrdU incorporation (Figure 4.18C). One major reason for this observation could be that other Notch homologs are more important in regulating the proliferative potential of PASMCs. Indeed, Notch3 has been described to be essential for aberrant proliferation of PASMCs in IPAH patients [147].

It can be inferred from these results that despite expression of Notch1 in PASMCs, its upregulation seen in IPAH patients and Hox+Su5416 rats can be attributed to functional activity in endothelial cells. These findings provide substantial evidence to our hypothesis of involvement of Notch1 in formation of plexiform lesions by contributing to endothelial proliferation and survival.

\subsection{Pharmacological inhibition of Notch signaling inhibits hPASMCs and hPAECs proliferation}

Dysregulated Notch signaling has been indicated in numerous human diseases, including broad range of cancers [141]. This has led to rapid development of $\gamma$ secretase inhibitors (GSIs) as potent therapeutic option. Inhibition of Notch signaling achieved by GSIs has been extensively used in various studies utilizing cell lines and animal models for different cancers [146, 184, 185] establishing potential utility of GSI based treatments. Furthermore, GSIs are being evaluated in clinical trials for breast cancer [143]. Though it should be noted that GSI based therapy could also be potentially relevant for developmental, vascular and cardiac conditions associated with Notch pathway malfunction, including pulmonary hypertension.

In severe PAH, two of the major histological findings are medial wall thickening due to SMC proliferation and plexiform lesions consisting of hyperproliferative endothelial cells $[7,8,16]$. Several reports have shown that targeting the medial wall thickening can be beneficial for PAH and can even, reverse the remodeling observed in PAH patients and rodent models [33, 48, 82]. Plexiform lesions are known to be pathological hallmark of PAH of different etiologies since long 
$[186,187]$; however, targeting strategies couldn't be formulated due to inability of any classical animal model to depict this patho-histological feature. Development of Hox+Su5416 model, characterized by medial remodeling along with plexiform lesions [155], has led the investigators to develop strategies focusing on these lesions $[155,156]$.

Based on our results, we demonstrated that increased Notch1 expression and activity is associated with endothelial proliferation occurring in plexiform lesions. In order to examine the relevance of GSIs as a therapy in experimental PH, we used DBZ ( $\gamma$ secretase inhibitor, GSI) for our in vitro studies. Hence, the effect of Notch signaling inhibitor, DBZ was studied on proliferation of hPAECs. DBZ was able to significantly reduce growth factor induced proliferation of endothelial cells (Figure 4.20A). At the similar concentrations, it further effectively reduced VEGF (important growth factor for ECs) induced proliferation (Figure 4.20B). In fact, crosstalk between VEGF and Notch signaling has already been established. VEGF was shown to increase expression of Notch receptors and ligands in endothelial cells [131]. Additionally, VEGF induced postnatal angiogenesis involving endothelial proliferation and migration is mediated via $y$ secretase and Notch1 activation [166]. Data from recent work dissecting Notch3 signaling in PAH, revealed that the GSI, DAPT was able to reduce the proliferation of PASMCs obtained from patients with IPAH [147]. On similar line, we investigated the effect of DBZ on proliferation of hPASMCs. Interestingly, serum induced proliferation of hPASMCs was effectively reduced by DBZ (Figure 4.21A). Moreover, DBZ reduced PDGF induced proliferation of hPASMCs (Figure 4.21B). PDGF is a potent mitogen for SMCs [45] and known to be an important player in pathogenesis of PAH $[46,47]$. Effect of Notch signaling inhibitor on PDGF-BB induced proliferation could be either due to direct effect of Notch on cell proliferation markers or due to cross talk with PDGF signaling. Indeed, Notch3 is shown to regulate PDGF receptor $\beta$ expression in vSMCs [135].

As observed from our results, DBZ was convincingly able to reduce proliferation of both hPAECs and hPASMCs; hence, using GSI could provide a therapeutic option in experimental PH.

\subsection{Conclusion}

In our study, we demonstrated an upregulation of Notch1 expression and cleavage in lungs and pulmonary arteries from IPAH patients. Similar upregulation was also observed in lungs from Hypoxia+Su5416 experimental model of PH. These screening results were further supported by stronger Notch1 immunoreactivity observed in endothelial cells of pulmonary arteries in lungs 
from IPAH patients in comparison to donors.

In vitro loss of function studies using Notch1 siRNA showed a reduction in proliferation of hPAECs, accompanied by increased apoptosis of the cells. On the other hand, Notch1ICD constitutive activation led to significant increase in proliferation and promoted survival of hPAECs. Although this study did not identify the complete signaling pathway downstream of the Notch1ICD translocation, leading to proliferation and survival of human pulmonary arterial endothelial cells, however, we identified two major proteins, p21 and Bcl-2, modulated by Notch1 (Figure 5.1). Additionally, Notch1 knockdown or constitutive activation did not have any effect on proliferation of hPASMCs, supporting endothelial specific effects of Notch1. Last but not the least, inhibition of Notch signaling in vitro by GSI, DBZ, effectively attenuated proliferation in both, hPAECs and hPASMCs, providing us with a platform for evaluating GSIs as a therapeutic option in experimental $\mathrm{PH}$ in future.

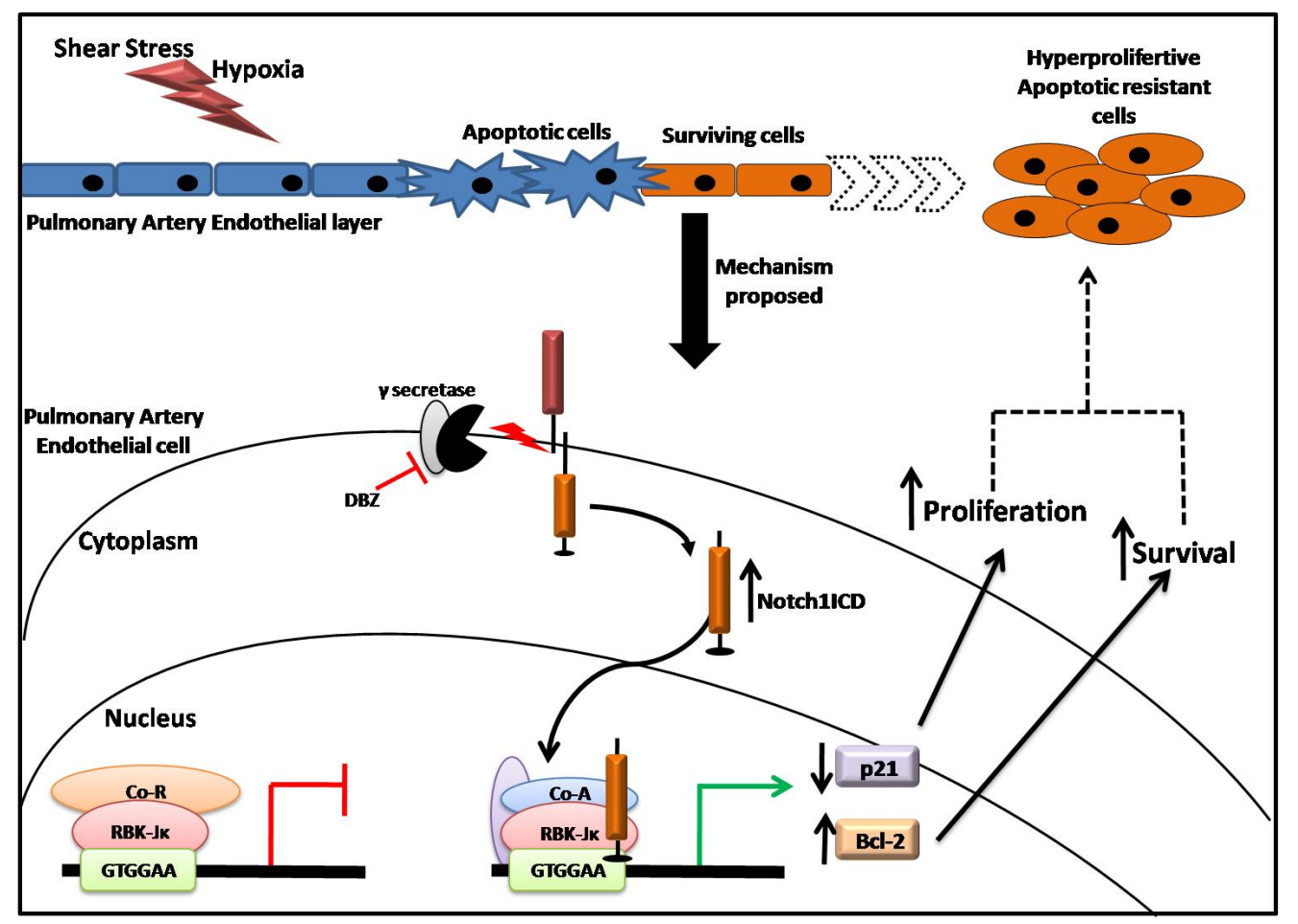

Figure5.1 Schematic representation of proposed role of Notch1 in endothelial proliferation and survival in $\mathrm{PH}$.

To the best of our knowledge, this is the first study demonstrating a role of Notch1 in vascular remodeling; essentially in endothelial proliferation and survival. Somatic mutations in BMPR2 rand BAX genes have been demonstrated in plexiform lesions in patients with IPAH [170], which may provide a growth advantage to altered endothelial cells. However, BMPRII mutations 
are observed only in $40-50 \%$ of familial PAH and not all plexiform lesions harbour somatic mutations. Hence, other molecular factors could be involved in acquiring hyperproliferative and antiapoptotic phenotype of endothelial cells in patients with severe $\mathrm{PH}$. Based on our studies, it is compelling to speculate that Notch1 could qualify as one such molecular mediator and hence, modulation of Notch1 signaling in the endothelium may be beneficial for patients with pulmonary hypertension.

\subsection{Future Outlook}

Taken together, our data puts forward a role of endothelial Notch1 signaling in vascular remodeling, specifically aberrant endothelial proliferation associated with $\mathrm{PAH}$. There are still certain areas that need future investigation. Further studies regarding the underlying cause of Notch1 upregulation in pulmonary artery endothelial cells are needed. It would be interesting to know whether the increase observed is a direct outcome of shear stress. It has not been proven though speculated that shear stress may lead to conformational changes in Notch receptors that increase their infinity towards proteases. Additionally, a possiblility of increased expression of Notch ligands leading to elevated notch cleavage and signaling cannot be neglected. It is further important to elucidate whether Notch1 directly has an effect on the expression of p21 and Bcl-2 or the effect is mediated via Hes and Hey family of genes (known target genes of Notch signaling pathway).

Most importantly, our findings need to be strengthened in vivo, in order to confirm a possibility of therapeutic targeting of Notch signaling in patients with $\mathrm{PAH}$. To test our concept in vivo, two approaches could be employed. To precisely evaluate the role of Notch1 in experimental model of $\mathrm{PH}$, Notch1 can be knocked down in Hox+Su5416 rats using adenoviral vectors carrying Notch1 shRNA. The effects of knockdown on pulmonary vascular remodeling can be evaluated by measuring various hemodynamic parameters. Another approach could be treatment of Hox+Su5416 rats with GSI, to analyze the effect of Notch inhibition on pulmonary vascular remodeling. Though GSIs inhibit the complete Notch signaling, they are the only available class of therapeutic inhibitors of Notch receptors. Generally, inhibition of complete Notch signaling via GSIs has been reported to result in off target gastrointestinal side effects. However, intratracheal nebulisation as a mode of delivery might provide a chance to target notch signaling in $\mathrm{PH}$ within acceptable levels of toxicity. In pulmonary hypertension, Notch3 has been previously reported to regulate proliferation of PASMCs in PAH [147] and we demonstrate importance of Notch1 in 
mediating endothelial proliferation and apoptosis. Hence, the potential use of blocking the Notch signaling by GSI treatment to target two important physio-pathological features of vascular remodeling in $\mathrm{PAH}$ is very exciting. 


\section{SUMMARY}

Pulmonary Hypertension is a disease condition characterized by elevated pulmonary vascular resistance which is an outcome of excessive vascular remodeling in pulmonary arteries involving deregulated proliferation of cells in all three layers of the vessels; intima, media as well as adventitia. New therapeutic options targeting vascular remodeling process are being developed and some are curently under clinical trials. Current evidence strongly supports involvement of Notch signaling in vascular homeostasis and injury. Recently, the impact of Notch3 and its target gene, Hes5 on increased proliferation of PASMCs in PAH patients has been described. However, the role of other Notch receptors and ligands has not been studied. This led us to investigate the expression and potential contribution of Notch signaling pathway in pathogenesis of $\mathrm{PH}$.

Interestingly, we observed an increased expression of Notch1 in lungs and pulmonary arteries from patients with IPAH. In line with this finding, increased Notch1 immunoreactivity was detected in intima of pulmonary arteries in lung tissues from IPAH patients as compared to donors. Furthermore, similar upregulation was observed in lungs from Hypoxia+Su5416 rats as compared to healthy rats. We demonstrate for the first time an upregulation of Notch1 in pulmonary hypertension patients. Additionally, absence of this increase in other two well established experimental models depicting only medial hypertrophy phenotype, monocrotaline rat model and chronic hypoxia mice model, led us to hypothesize that Notch1 may contribute substantially to pathogenesis of plexiform lesions.

In vasculature, Notch1 expression is observed in both smooth muscle cells and endothelial cells. We demonstrated a decrease in proliferation and increase in apoptosis of human pulmonary artery endothelial cells (hPAECs) with siRNA mediated Notch1 inhibition. In contrast, an increase in proliferation and survival of hPAECs was observed on constitutive Notch1 activation. These effects were associated with modulation of expression of cell cycle inhibitor, p21 and apoptosis inhibitor, Bcl-2. Interestingly, Notch1 inhibition or activation did not have any effect on proliferation of human pulmonary artery smooth muscle cells (hPASMCs), indicating, endothelial cell specific effect of Notch1 signaling in vasculature.

The anti-proliferative and pro-apoptotic effect of Notch1 signaling inhibition, observed in our results, puts forward Notch inhibition as a therapeutic option for treatment of pulmonary 
hypertension. Presently, $y$ secretase inhibitors (GSIs) are the only clinically available inhibitors targeting this pathway. GSIs inhibit the $\gamma$ secretase enzyme, responsible for cleavage of Notch receptors. In order to examine the relevance of GSIs as a therapy in experimental PH, we used DBZ (a commercially available GSI) for our in vitro studies. Inhibition of Notch signaling by employing DBZ effectively reduced expression of Hes-1 (target gene of Notch signaling pathway) and attenuated proliferation of both, hPAECs and hPASMCs.

Taken together, this study puts forward a role of Notch1 signaling in regulation of proliferation and apoptosis of endothelial cells which might contribute to formation of plexiform lesions observed in pulmonary hypertension. Nonetheless, our study needs to be further substantiated with in vivo studies using GSI in Hox+Su5416 rat model of PH. 


\section{ZUSAMMENFASSUNG}

Pulmonale Hypertonie (PH) ist eine Erkrankung der Lungengefäße, die durch einen erhöhten pulmonalen Gefäßwiderstand charakterisiert ist. Strukturelle Umbauprozesse in den pulmonalen Gefäßen finden in allen drei Gefäßschichten -Intima, Media und Adventitia- statt ist und sind durch dysregulierte Proliferation/Apoptose von verschiedenen Gefäß-Zellen ausgezeichnet. Neue therapeutische Optionen, die den Prozess des Gefäßumbaus aufhalten oder umzukehren, befinden sich momentan in der Entwicklung oder in klinischen Studien. Der derzeitige Stand der wissenschaftlichen Erkenntnis legt eine Involvierung von NotchSignalübertragungswegen in die vaskulare Homöostase nahe. So konnte unlängst der Einfluss von Notch3 und dessen Zielgen Hes5 auf die erhöhte Proliferation von pulmonalarteriellen glatten Muskelzellen (PASMCS) in Patienten mit pulmonalarterieller Hypertonie (PAH) nachgewiesen werden. Der jeweilige Einfluss anderer Notch-Rezeptoren sowie deren Liganden wurde jedoch noch nicht beschrieben. In Folge dessen untersuchten wir die Expression und den potenziellen Beitrag von Notch-Signalwegen in der Pathogenese von PH.

Interessanterweise konnten wir eine erhöhte Expression von Notch1, sowohl in Lungen, als auch in Pulmonalarterien von IPAH-Patienten beobachten. In Übereinstimmung mit dieser Erkenntnis wurde eine erhöhte Notch1-Immunreaktivität in der Intima von Pulmonalarterien in Lungengewebe von IPAH-Patienten verglichen mit Donoren detektiert. Des Weiteren wurde eine ähnlich erhöhte Expression in Lungen von Ratten mit PH (Hypoxia+Su5416 Modell) verglichen mit gesunden Ratten beobachtet. Damit demonstrieren wir zum ersten Mal eine Hochregulation von Notch1 in Patienten mit pulmonaler Hypertonie. Zusätzlich lässt die Abwesenheit dieser Hochregulation in zwei weiteren gut etablierten experimentellen Modellen mit medialer Hypertrophie, dem Monocrotalin-injizierten Ratten-Model und dem Maus-Modell mit chronischer Hypoxie, die Hypothese zu, dass Notch1 substanziell zu der Pathogenese von plexiformen Läsionen beiträgt.

Im Gefäßsystem selbst wird Notch1-Expression sowohl in der glatten Muskulatur als auch in den Endothelzellen beobachtet. Wir konnten eine erniedrigte Proliferation und eine erhöhte Apoptoserate in humanen pulmonal-arteriellen Endothelzellen (hPAECs) nach erfolgter siRNAvermittelter Inhibition von Notch1 demonstrieren. Im Gegensatz dazu konnte eine erhöhte Proliferation und Überlebensrate von hPAECs nach konstitutiver Notch1-Aktivierung beobachtet werden. Diese Effekte waren mit modulierter Expression des Zellzyklus-Inhibitors p21 und des Apoptose-Inhibitors Bcl-2 assoziiert. Interessanterweise zeigten weder Notch1-Inhibition noch - 
Aktivierung einen Effekt auf die Proliferation von humanen pulmonal-arteriellen glatten Muskelzellen (hPASMCs), was einen Endothel-spezifischen Effekt von Notch1-Signalwegen im Gefäßsystem indiziert.

Die beobachteten anti-proliferativen und pro-apoptotischen Effekte nach Inhibition von Notch1-Signalwegen kennzeichnen die Notch1-Inhibition als therapeutische Option für pulmonale Hypertonie. Derzeitig sind $\gamma$-Sekretase-Inhibitoren (GSIs) die einzigen klinisch verfügbaren Inhibitoren für diesen Signalweg. GSIs inhibieren das Enzym y-Sekretase, das verantwortlich für die Spaltung von Notch-Rezeptoren ist. Um die Relevanz von GSIs als Therapie für experimentelle pulmonale Hypertonie zu untersuchen, haben wir den kommerziell verfügbaren GSI "DBZ“ in unseren in vitro Studien appliziert. Inhibition des Notch-Signalwegs durch die Anwendung von DBZ reduzierte effektiv die Expression von Hes1, das Zielgen des Notch-Signalwegs, und schwächte die Proliferation von sowohl hPAECs als auch von hPASMCs ab.

Zusammengefasst zeigt diese Studie die Rolle von Notch1-Signalwegen in Proliferation und Apoptose von Endothelzellen, die zur Entstehung von plexiformen Läsionen, wie sie bei der pulmonalen Hypertonie beobachtet werden, beitragen können. Gleichwohl muss unsere Studie weiter durch in vivo Studien mit Applikation von GSI in experimentellen Modellen der PH untermauert werden. 


\section{APPENDIX}

Table 1 App: List of primers used for real time PCRs

\begin{tabular}{|c|c|c|c|c|}
\hline Gene & Species & Primers & $\begin{array}{c}\text { Annealing } \\
\text { ('C) }\end{array}$ & Product Size \\
\hline \multirow[t]{2}{*}{ Notch1 } & Rat & F:GAGATGCTCCCAGCCAAGT & 60 & 174 \\
\hline & & R: CCATGGTCCACAACATAGCA & & \\
\hline \multirow[t]{2}{*}{ Notch2 } & Rat & F: GTGAAGATGCCCGCTCTG & 60 & 151 \\
\hline & & R: CTGTGCCGTTGTGGTAGGTA & & \\
\hline \multirow[t]{2}{*}{ Notch3 } & Rat & F: CCTTGCCGGAACGGTGCCAA & 60 & 184 \\
\hline & & R: CCGTGTAGCCTGGCGCACAA & & \\
\hline \multirow[t]{2}{*}{ Notch4 } & Rat & F: CTGGATTCGAGGGCAAGTTA & 60 & 194 \\
\hline & & R: GGGTACAGGTTCCATGGTTG & & \\
\hline \multirow[t]{2}{*}{ DLL1 } & Rat & F: GGAGAGAGGGGAGAAGATG & 60 & 108 \\
\hline & & R: CCGGTTTGTCACAATATCCA & & \\
\hline \multirow[t]{2}{*}{ DLL3 } & Rat & F: TGAAACCTGGAGAGAGCAGC & 60 & 147 \\
\hline & & R: GAGAAGTGCAACTCCCATGC & & \\
\hline \multirow[t]{2}{*}{ DLL4 } & Rat & F: GAATGTATCCCCCACAATGG & 60 & 158 \\
\hline & & R: CCACTGTTGGAGCAAGTTGA & & \\
\hline \multirow[t]{2}{*}{ Jagged1 } & Rat, Mouse & F: CAGGGTCTACGCCTGTCATC & 60 & 118 \\
\hline & & R: AGCAAAGTGTAGGACCTCGG & & \\
\hline \multirow[t]{2}{*}{ Jagged2 } & Rat, Mouse & F: GGATGTAATTTGCTCCACGG & 60 & 157 \\
\hline & & R: CCCAGTTGGTCTCACAGTCA & & \\
\hline \multirow[t]{2}{*}{ PBGD } & Rat & F: ATGTCCGGTAACGGCGGC & 58 & 135 \\
\hline & & R: CAAGGTTTTCAGCATCGCTAC & & \\
\hline \multirow[t]{2}{*}{ Notch1 } & Mouse & F: GAGATGCTCCCAGCCAAGT & 60 & 143 \\
\hline & & R: TCTTACACGGTGTGCTGAGG & & \\
\hline \multirow[t]{2}{*}{ Notch2 } & Mouse & F: TTACCTACCACAACGGCACA & 60 & 175 \\
\hline & & R: TCCTCTCCTGTGAATCCTGG & & \\
\hline \multirow[t]{2}{*}{ Notch3 } & Mouse & F: GGGTCCGTCGTGAGCTGGGT & 60 & 198 \\
\hline & & R: GCGGCTCTCCTCGCACATCC & & \\
\hline \multirow[t]{2}{*}{ Notch4 } & Mouse & F: TGCCAGGATAATGTGAACC & 60 & 175 \\
\hline & & R: AGGTACAGGTTCCGTGGTTG & & \\
\hline \multirow[t]{2}{*}{ DLL1 } & Mouse & F:AGGTTGCTCTGTGTTCTGCC & 60 & 169 \\
\hline & & R: CTCCCCTGGTTTGTCACAGT & & \\
\hline
\end{tabular}


APPENDIX

\begin{tabular}{|c|c|c|c|c|}
\hline \multirow[t]{2}{*}{ DLL3 } & \multirow[t]{2}{*}{ Mouse } & F: CCAGTAGCTGCCTGAACTCC & \multirow[t]{2}{*}{60} & \multirow[t]{2}{*}{168} \\
\hline & & R: CACATCGAAGCCCGTAGAAT & & \\
\hline \multirow[t]{2}{*}{ DLL4 } & \multirow[t]{2}{*}{ Mouse } & F: GTGCAATGAATGTATCCC & \multirow[t]{2}{*}{60} & \multirow[t]{2}{*}{185} \\
\hline & & R: TGCAGGTATAACCCTTTGGC & & \\
\hline \multirow[t]{2}{*}{ PBGD } & \multirow[t]{2}{*}{ Mouse } & F: CTTCTGCAGACACCAGGGGA & \multirow[t]{2}{*}{58} & \multirow[t]{2}{*}{104} \\
\hline & & R: GCCGTTTTCTTCCGCGGTT & & \\
\hline \multirow[t]{2}{*}{ Notch1 } & \multirow[t]{2}{*}{ Human } & F: GTGAGACCTGCCTGAATGG & \multirow[t]{2}{*}{60} & \multirow[t]{2}{*}{101} \\
\hline & & R: GTTGGGGTCCTGGCATC & & \\
\hline \multirow[t]{2}{*}{ Notch2 } & \multirow[t]{2}{*}{ Human } & F: ATGACTGCCCTAACCACAGG & \multirow[t]{2}{*}{60} & \multirow[t]{2}{*}{119} \\
\hline & & R: TTCATCCACATCCTCTGTGC & & \\
\hline \multirow[t]{2}{*}{ Notch3 } & \multirow[t]{2}{*}{ Human } & F: GTGTGCAAATGGAGGTCGTT & \multirow[t]{2}{*}{60} & \multirow[t]{2}{*}{149} \\
\hline & & R: CCACTGAACTCTGGCAGACA & & \\
\hline \multirow[t]{2}{*}{ Notch4 } & \multirow[t]{2}{*}{ Human } & F: CACGTGAACCCATGTGAGTC & \multirow[t]{2}{*}{60} & \multirow[t]{2}{*}{126} \\
\hline & & R: GCATCGAGTTCCTTTGAGC & & \\
\hline \multirow[t]{2}{*}{ DLL1 } & \multirow[t]{2}{*}{ Human } & F: AGAGGGCTGCTCCGTTTT & \multirow[t]{2}{*}{60} & \multirow[t]{2}{*}{123} \\
\hline & & R: CAGATCGGCTCTGTGCAGTA & & \\
\hline \multirow[t]{2}{*}{ DLL3 } & Human & F: GCTCGAGGACGAATGTGAG & 60 & 106 \\
\hline & & R: AGTCCAGCCCTCTAGGCATC & & \\
\hline DLL4 & Human & F: GCACTCCCTGGCAATGTACT & 60 & 145 \\
\hline & & R: CGACAGGTGCAGGTGTAGC & & \\
\hline Jagged1 & Human & F: AACACCTTCAACCTCAAGGC & 60 & 119 \\
\hline & & R: TCATTACTGGAATCCCACGC & & \\
\hline Jagged2 & Human & F: AGGGTGTAATTTGCTCCACG & 60 & 100 \\
\hline & & R: GGGTAGGGGACACACTCAT & & \\
\hline Hes1 & Human & F: GCAGATGACGGCTGCGCTGA & 60 & 195 \\
\hline & & R: GGCTGCCCGGGGTAGGTCAT & & \\
\hline PBGD & Human & F: CAGGAGTCAGACTGTAGG & 58 & 179 \\
\hline & & R: АCTCTCATCTTTGGGCTGTTTTTC & & \\
\hline
\end{tabular}

Table 2 App: List of primary antibodies

\begin{tabular}{clccc}
\hline Antibody & Source & $\begin{array}{c}\text { Dilution } \\
\text { WB }\end{array}$ & $\begin{array}{c}\text { Dilution } \\
\text { IHC/ICC }\end{array}$ & Company \\
\hline Notch1 & Rabbit & $1: 200$ & & Santa Cruz \\
$\boldsymbol{\alpha}$ smooth muscle & Rabbit & & $1: 300$ & Sigma \\
\hline
\end{tabular}




\begin{tabular}{|c|c|c|c|}
\hline actin & & & \\
\hline Notch2 & Rabbit & $1: 200$ & Santa Cruz \\
\hline Notch3 & Rabbit & $1: 200$ & Santa Cruz \\
\hline Notch4 & Rabbit & $1: 200$ & Santa Cruz \\
\hline DLL1 & Rabbit & $1: 200$ & Santa Cruz \\
\hline DLL3 & Rabbit & $1: 200$ & Santa Cruz \\
\hline DLL4 & Rabbit & $1: 200$ & Santa Cruz \\
\hline Jagged1 & Rabbit & $1: 200$ & Santa Cruz \\
\hline Jagged2 & Rabbit & $1: 200$ & Santa Cruz \\
\hline $\begin{array}{l}\text { Von-willibrand } \\
\text { factor }\end{array}$ & Mouse & $1: 300$ & Dako \\
\hline ß-actin & Mouse & $1: 5000$ & Sigma Aldrich \\
\hline GAPDH & Mouse & $1: 5000$ & Novus \\
\hline p21 & Mouse & $1: 1000$ & BD Transduction \\
\hline Cyclin D1 & Rabbit & $1: 200$ & Santa Cruz \\
\hline Cyclin B1 & Rabbit & $1: 200$ & Santa Cruz \\
\hline p27 & Mouse & $1: 200$ & Santa Cruz \\
\hline Bax & Rabbit & $1: 1000$ & Cell Signaling \\
\hline Bcl-2 & Rabbit & $1: 1000$ & Cell Signaling \\
\hline
\end{tabular}

Table3 App: List of secondary antibodies

\begin{tabular}{ccl}
\hline Antibody & $\begin{array}{c}\text { Dilution } \\
\text { WB }\end{array}$ & Company \\
\hline HRP conjugated anti-rabbit IgG & $1: 40000$ & Sigma Aldrich \\
HRP conjugated anti-mouse IgG & $1: 30000$ & Sigma Aldrich \\
\hline
\end{tabular}




\section{REFERENCES}

1. Simonneau, G., et al., Clinical classification of pulmonary hypertension. J Am Coll Cardiol, 2004. 43(12 Suppl S): p. 5S-12S.

2. Gaine, S.P. and L.J. Rubin, Primary pulmonary hypertension. Lancet, 1998. 352(9129): p. 719-25.

3. McLaughlin, V.V., A. Shillington, and S. Rich, Survival in primary pulmonary hypertension: the impact of epoprostenol therapy. Circulation, 2002. 106(12): p. 1477-82.

4. Dresdale, D.T., M. Schultz, and R.J. Michtom, Primary pulmonary hypertension. I. Clinical and hemodynamic study. Am J Med, 1951. 11(6): p. 686-705.

5. Fishman, A.P., Clinical classification of pulmonary hypertension. Clin Chest Med, 2001. 22(3): p. 385-91, vii.

6. Simonneau, G., et al., Updated clinical classification of pulmonary hypertension. J Am Coll Cardiol, 2009. 54(1 Suppl): p. S43-54.

7. Humbert, M., et al., Cellular and molecular pathobiology of pulmonary arterial hypertension. J Am Coll Cardiol, 2004. 43(12 Suppl S): p. 13S-24S.

8. Mandegar, M., et al., Cellular and molecular mechanisms of pulmonary vascular remodeling: role in the development of pulmonary hypertension. Microvasc Res, 2004. 68(2): p. 75-103.

9. Budhiraja, R., R.M. Tuder, and P.M. Hassoun, Endothelial dysfunction in pulmonary hypertension. Circulation, 2004. 109(2): p. 159-65.

10. Christman, B.W., et al., An imbalance between the excretion of thromboxane and prostacyclin metabolites in pulmonary hypertension. N Engl J Med, 1992. 327(2): p. 70-5.

11. Rich, S., Clinical insights into the pathogenesis of primary pulmonary hypertension. Chest, 1998. 114(3 Suppl): p. 237S-241S.

12. Johnson, S.R., J.T. Granton, and S. Mehta, Thrombotic arteriopathy and anticoagulation in pulmonary hypertension. Chest, 2006. 130(2): p. 545-52.

13. Schermuly, R.T., et al., Mechanisms of disease: pulmonary arterial hypertension. Nat Rev Cardiol, 2011. 8(8): p. 443-55.

14. Chaouat, A., E. Weitzenblum, and T. Higenbottam, The role of thrombosis in severe pulmonary hypertension. Eur Respir J, 1996. 9(2): p. 356-63.

15. Archer, S.L., E.K. Weir, and M.R. Wilkins, Basic science of pulmonary arterial hypertension for clinicians: new concepts and experimental therapies. Circulation, 2010. 121(18): p. 2045-66.

16. Tuder, R.M., et al., Pathology of pulmonary hypertension. Clin Chest Med, 2007. 28(1): p. 23-42, vii.

17. Yi, E.S., et al., Distribution of obstructive intimal lesions and their cellular phenotypes in chronic pulmonary hypertension. A morphometric and immunohistochemical study. Am J Respir Crit Care Med, 2000. 162(4 Pt 1): p. 1577-86.

18. Abe, K., et al., Formation of plexiform lesions in experimental severe pulmonary arterial hypertension. Circulation, 2010. 121(25): p. 2747-54.

19. Hopkins, N. and P. McLoughlin, The structural basis of pulmonary hypertension in chronic lung disease: remodelling, rarefaction or angiogenesis? J Anat, 2002. 201(4): p. 335-48.

20. Stenmark, K.R., et al., Role of the adventitia in pulmonary vascular remodeling. Physiology (Bethesda), 2006. 21: p. 134-45.

21. Durmowicz, A.G. and K.R. Stenmark, Mechanisms of structural remodeling in chronic pulmonary hypertension. Pediatr Rev, 1999. 20(11): p. e91-e102.

22. Olschewski, H., et al., Prostacyclin and its analogues in the treatment of pulmonary 
hypertension. Pharmacol Ther, 2004. 102(2): p. 139-53.

23. Geraci, M.W., et al., Pulmonary prostacyclin synthase overexpression in transgenic mice protects against development of hypoxic pulmonary hypertension. J Clin Invest, 1999. 103(11): p. 1509-15.

24. Hoshikawa, Y., et al., Prostacyclin receptor-dependent modulation of pulmonary vascular remodeling. Am J Respir Crit Care Med, 2001. 164(2): p. 314-8.

25. Wilkins, M.R., et al., Phosphodiesterase inhibitors for the treatment of pulmonary hypertension. Eur Respir J, 2008. 32(1): p. 198-209.

26. Giaid, A. and D. Saleh, Reduced expression of endothelial nitric oxide synthase in the lungs of patients with pulmonary hypertension. N Engl J Med, 1995. 333(4): p. 214-21.

27. Bowers, R., et al., Oxidative stress in severe pulmonary hypertension. Am J Respir Crit Care Med, 2004. 169(6): p. 764-9.

28. Zakrzewicz, D. and O. Eickelberg, From arginine methylation to ADMA: a novel mechanism with therapeutic potential in chronic lung diseases. BMC Pulm Med, 2009. 9: p. 5.

29. Perros, F., P. Dorfmuller, and M. Humbert, Current insights on the pathogenesis of pulmonary arterial hypertension. Semin Respir Crit Care Med, 2005. 26(4): p. 355-64.

30. Petkov, V., et al., Vasoactive intestinal peptide as a new drug for treatment of primary pulmonary hypertension. J Clin Invest, 2003. 111(9): p. 1339-46.

31. Giaid, A., et al., Expression of endothelin-1 in the lungs of patients with pulmonary hypertension. N Engl J Med, 1993. 328(24): p. 1732-9.

32. Li, H., et al., Enhanced endothelin-1 and endothelin receptor gene expression in chronic hypoxia. J Appl Physiol, 1994. 77(3): p. 1451-9.

33. Pullamsetti, S.S. and R.T. Schermuly, Endothelin receptor antagonists in preclinical models of pulmonary hypertension. Eur J Clin Invest, 2009. 39 Suppl 2: p. 3-13.

34. Hirata, Y., et al., Endothelin receptor subtype $B$ mediates synthesis of nitric oxide by cultured bovine endothelial cells. J Clin Invest, 1993. 91(4): p. 1367-73.

35. Naeije, R. and S. Eddahibi, Serotonin in pulmonary arterial hypertension. Am J Respir Crit Care Med, 2004. 170(3): p. 209-10.

36. Dempsie, Y. and M.R. MacLean, Pulmonary hypertension: therapeutic targets within the serotonin system. Br J Pharmacol, 2008. 155(4): p. 455-62.

37. Herve, P., et al., Increased plasma serotonin in primary pulmonary hypertension. Am J Med, 1995. 99(3): p. 249-54.

38. Eddahibi, S., et al., Attenuated hypoxic pulmonary hypertension in mice lacking the 5hydroxytryptamine transporter gene. J Clin Invest, 2000. 105(11): p. 1555-62.

39. Eddahibi, S., et al., Serotonin transporter overexpression is responsible for pulmonary artery smooth muscle hyperplasia in primary pulmonary hypertension. J Clin Invest, 2001. 108(8): p. 1141-50.

40. Geraci, M.W., et al., Gene expression patterns in the lungs of patients with primary pulmonary hypertension: a gene microarray analysis. Circ Res, 2001. 88(6): p. 555-62.

41. $\mathrm{Yu}, \mathrm{Y}$., et al., Enhanced expression of transient receptor potential channels in idiopathic pulmonary arterial hypertension. Proc Natl Acad Sci U S A, 2004. 101(38): p. 13861-6.

42. Lane, K.B., et al., Heterozygous germline mutations in BMPR2, encoding a TGF-beta receptor, cause familial primary pulmonary hypertension. Nat Genet, 2000. 26(1): p. 81-4.

43. Thomson, J.R., et al., Sporadic primary pulmonary hypertension is associated with germline mutations of the gene encoding BMPR-II, a receptor member of the TGF-beta family. J Med Genet, 2000. 37(10): p. 741-5.

44. Trembath, R.C., et al., Clinical and molecular genetic features of pulmonary hypertension in patients with hereditary hemorrhagic telangiectasia. N Engl J Med, 2001. 345(5): p. 325-34. 
45. Andrae, J., R. Gallini, and C. Betsholtz, Role of platelet-derived growth factors in physiology and medicine. Genes Dev, 2008. 22(10): p. 1276-312.

46. Perros, F., et al., Platelet-derived growth factor expression and function in idiopathic pulmonary arterial hypertension. Am J Respir Crit Care Med, 2008. 178(1): p. 81-8.

47. Balasubramaniam, V., et al., Role of platelet-derived growth factor in vascular remodeling during pulmonary hypertension in the ovine fetus. Am J Physiol Lung Cell Mol Physiol, 2003. 284(5): p. L826-33.

48. Schermuly, R.T., et al., Reversal of experimental pulmonary hypertension by PDGF inhibition. J Clin Invest, 2005. 115(10): p. 2811-21.

49. Tuder, R.M., et al., Expression of angiogenesis-related molecules in plexiform lesions in severe pulmonary hypertension: evidence for a process of disordered angiogenesis. J Pathol, 2001. 195(3): p. 367-74.

50. Jones, P.L., J. Crack, and M. Rabinovitch, Regulation of tenascin- $C$, a vascular smooth muscle cell survival factor that interacts with the alpha $v$ beta 3 integrin to promote epidermal growth factor receptor phosphorylation and growth. J Cell Biol, 1997. 139(1): p. 279-93.

51. Merklinger, S.L., et al., Epidermal growth factor receptor blockade mediates smooth muscle cell apoptosis and improves survival in rats with pulmonary hypertension. Circulation, 2005. 112(3): p. 423-31.

52. Benisty, J.I., et al., Elevated basic fibroblast growth factor levels in patients with pulmonary arterial hypertension. Chest, 2004. 126(4): p. 1255-61.

53. Izikki, M., et al., Endothelial-derived FGF2 contributes to the progression of pulmonary hypertension in humans and rodents. J Clin Invest, 2009. 119(3): p. 512-23.

54. Hassoun, P.M., et al., Inflammation, growth factors, and pulmonary vascular remodeling. J Am Coll Cardiol, 2009. 54(1 Suppl): p. S10-9.

55. McMurtry, M.S., et al., Gene therapy targeting survivin selectively induces pulmonary vascular apoptosis and reverses pulmonary arterial hypertension. J Clin Invest, 2005. 115(6): p. 1479-91.

56. Pullamsetti, S.S., et al., Inflammation, immunological reaction and role of infection in pulmonary hypertension. Clin Microbiol Infect, 2011. 17(1): p. 7-14.

57. Savale, L., et al., Impact of interleukin-6 on hypoxia-induced pulmonary hypertension and lung inflammation in mice. Respir Res, 2009. 10: p. 6.

58. Steiner, M.K., et al., Interleukin-6 overexpression induces pulmonary hypertension. Circ Res, 2009. 104(2): p. 236-44, 28p following 244.

59. Hagen, M., et al., Interaction of interleukin-6 and the BMP pathway in pulmonary smooth muscle. Am J Physiol Lung Cell Mol Physiol, 2007. 292(6): p. L1473-9.

60. Brock, M., et al., Interleukin-6 modulates the expression of the bone morphogenic protein receptor type II through a novel STAT3-microRNA cluster 17/92 pathway. Circ Res, 2009. 104(10): p. 1184-91.

61. Balabanian, K., et al., $C X(3) C$ chemokine fractalkine in pulmonary arterial hypertension. Am J Respir Crit Care Med, 2002. 165(10): p. 1419-25.

62. Sanchez, O., et al., Role of endothelium-derived CC chemokine ligand 2 in idiopathic pulmonary arterial hypertension. Am J Respir Crit Care Med, 2007. 176(10): p. 1041-7.

63. O'Callaghan, D.S., et al., Treatment of pulmonary arterial hypertension with targeted therapies. Nat Rev Cardiol, 2011. 8(9): p. 526-38.

64. Barst, R.J., et al., A comparison of continuous intravenous epoprostenol (prostacyclin) with conventional therapy for primary pulmonary hypertension. The Primary Pulmonary Hypertension Study Group. N Engl J Med, 1996. 334(5): p. 296-302. 
65. Voswinckel, R., et al., Inhaled treprostinil [corrected] for treatment of chronic pulmonary arterial hypertension. Ann Intern Med, 2006. 144(2): p. 149-50.

66. Olschewski, H., et al., Inhaled iloprost for severe pulmonary hypertension. N Engl J Med, 2002. 347(5): p. 322-9.

67. Barst, R.J., et al., Beraprost therapy for pulmonary arterial hypertension. J Am Coll Cardiol, 2003. 41(12): p. 2119-25.

68. Rubin, L.J., et al., Bosentan therapy for pulmonary arterial hypertension. N Engl J Med, 2002. 346(12): p. 896-903.

69. Barst, R.J., et al., Sitaxsentan therapy for pulmonary arterial hypertension. Am J Respir Crit Care Med, 2004. 169(4): p. 441-7.

70. Galie, N., et al., Ambrisentan for the treatment of pulmonary arterial hypertension: results of the ambrisentan in pulmonary arterial hypertension, randomized, double-blind, placebocontrolled, multicenter, efficacy (ARIES) study 1 and 2. Circulation, 2008. 117(23): p. 30109.

71. Iglarz, M., et al., Pharmacology of macitentan, an orally active tissue-targeting dual endothelin receptor antagonist. J Pharmacol Exp Ther, 2008. 327(3): p. 736-45.

72. Michelakis, E., et al., Oral sildenafil is an effective and specific pulmonary vasodilator in patients with pulmonary arterial hypertension: comparison with inhaled nitric oxide. Circulation, 2002. 105(20): p. 2398-403.

73. Galie, N., et al., Sildenafil citrate therapy for pulmonary arterial hypertension. N Engl J Med, 2005. 353(20): p. 2148-57.

74. Galie, N., et al., Tadalafil therapy for pulmonary arterial hypertension. Circulation, 2009. 119(22): p. 2894-903.

75. Leuchte, H.H., et al., Inhalation of vasoactive intestinal peptide in pulmonary hypertension. Eur Respir J, 2008. 32(5): p. 1289-94.

76. Evgenov, O.V., et al., NO-independent stimulators and activators of soluble guanylate cyclase: discovery and therapeutic potential. Nat Rev Drug Discov, 2006. 5(9): p. 755-68.

77. Schermuly, R.T., et al., Expression and function of soluble guanylate cyclase in pulmonary arterial hypertension. Eur Respir J, 2008. 32(4): p. 881-91.

78. Ghofrani, H.A., et al., Riociguat for chronic thromboembolic pulmonary hypertension and pulmonary arterial hypertension: a phase Il study. Eur Respir J, 2010. 36(4): p. 792-9.

79. Kuwano, K., et al., A long-acting and highly selective prostacyclin receptor agonist prodrug, 2-\{4-[(5,6-diphenylpyrazin-2-yl)(isopropyl)amino]butoxy\}-N-(methylsulfonyl)acetam ide (NS304), ameliorates rat pulmonary hypertension with unique relaxant responses of its active form, \{4-[(5,6-diphenylpyrazin-2-yl)(isopropyl)amino]butoxy\}acetic acid (MRE-269), on rat pulmonary artery. J Pharmacol Exp Ther, 2008. 326(3): p. 691-9.

80. Ghofrani, H.A., W. Seeger, and F. Grimminger, Imatinib for the treatment of pulmonary arterial hypertension. N Engl J Med, 2005. 353(13): p. 1412-3.

81. Souza, R., et al., Long term imatinib treatment in pulmonary arterial hypertension. Thorax, 2006. 61(8): p. 736.

82. Klein, M., et al., Combined tyrosine and serine/threonine kinase inhibition by sorafenib prevents progression of experimental pulmonary hypertension and myocardial remodeling. Circulation, 2008. 118(20): p. 2081-90.

83. Borggrefe, T. and F. Oswald, The Notch signaling pathway: transcriptional regulation at Notch target genes. Cell Mol Life Sci, 2009. 66(10): p. 1631-46.

84. Artavanis-Tsakonas, S., M.D. Rand, and R.J. Lake, Notch signaling: cell fate control and signal integration in development. Science, 1999. 284(5415): p. 770-6.

85. Kopan, R. and M.X. Ilagan, The canonical Notch signaling pathway: unfolding the activation 
mechanism. Cell, 2009. 137(2): p. 216-33.

86. Artavanis-Tsakonas, S., M.A. Muskavitch, and B. Yedvobnick, Molecular cloning of Notch, a locus affecting neurogenesis in Drosophila melanogaster. Proc Natl Acad Sci U S A, 1983. 80(7): p. 1977-81.

87. Kidd, S., T.J. Lockett, and M.W. Young, The Notch locus of Drosophila melanogaster. Cell, 1983. 34(2): p. 421-33.

88. Wharton, K.A., et al., Nucleotide sequence from the neurogenic locus notch implies a gene product that shares homology with proteins containing EGF-like repeats. Cell, 1985. 43(3 Pt 2): p. 567-81.

89. Ellisen, L.W., et al., TAN-1, the human homolog of the Drosophila notch gene, is broken by chromosomal translocations in T lymphoblastic neoplasms. Cell, 1991. 66(4): p. 649-61.

90. Weinmaster, G., V.J. Roberts, and G. Lemke, Notch2: a second mammalian Notch gene. Development, 1992. 116(4): p. 931-41.

91. Lardelli, M., J. Dahlstrand, and U. Lendahl, The novel Notch homologue mouse Notch 3 lacks specific epidermal growth factor-repeats and is expressed in proliferating neuroepithelium. Mech Dev, 1994. 46(2): p. 123-36.

92. Uyttendaele, H., et al., Notch4/int-3, a mammary proto-oncogene, is an endothelial cellspecific mammalian Notch gene. Development, 1996. 122(7): p. 2251-9.

93. Tien, A.C., A. Rajan, and H.J. Bellen, A Notch updated. J Cell Biol, 2009. 184(5): p. 621-9.

94. Bray, S.J., Notch signalling: a simple pathway becomes complex. Nat Rev Mol Cell Biol, 2006. 7(9): p. 678-89.

95. D'Souza, B., A. Miyamoto, and G. Weinmaster, The many facets of Notch ligands. Oncogene, 2008. 27(38): p. 5148-67.

96. Shimizu, K., et al., Mouse jagged1 physically interacts with notch2 and other notch receptors. Assessment by quantitative methods. J Biol Chem, 1999. 274(46): p. 32961-9.

97. Pintar, A., et al., The intracellular region of Notch ligands: does the tail make the difference? Biol Direct, 2007. 2: p. 19.

98. Jorissen, E. and B. De Strooper, Gamma-secretase and the intramembrane proteolysis of Notch. Curr Top Dev Biol, 2010. 92: p. 201-30.

99. Levy-Lahad, E., et al., Candidate gene for the chromosome 1 familial Alzheimer's disease locus. Science, 1995. 269(5226): p. 973-7.

100. De Strooper, B., et al., Deficiency of presenilin-1 inhibits the normal cleavage of amyloid precursor protein. Nature, 1998. 391(6665): p. 387-90.

101. Hansson, E.M., et al., Aph-1 interacts at the cell surface with proteins in the active gammasecretase complex and membrane-tethered Notch. J Neurochem, 2005. 92(5): p. 1010-20.

102. Wolfe, M.S., et al., Two transmembrane aspartates in presenilin-1 required for presenilin endoproteolysis and gamma-secretase activity. Nature, 1999. 398(6727): p. 513-7.

103. Tun, T., et al., Recognition sequence of a highly conserved DNA binding protein RBP-J kappa. Nucleic Acids Res, 1994. 22(6): p. 965-71.

104. Jarriault, S., et al., Signalling downstream of activated mammalian Notch. Nature, 1995. 377(6547): p. 355-8.

105. Fischer, A. and M. Gessler, Delta-Notch--and then? Protein interactions and proposed modes of repression by Hes and Hey bHLH factors. Nucleic Acids Res, 2007. 35(14): p. 458396.

106. Iso, T., L. Kedes, and Y. Hamamori, HES and HERP families: multiple effectors of the Notch signaling pathway. J Cell Physiol, 2003. 194(3): p. 237-55.

107. Satoh, Y., et al., Roles for c-Myc in self-renewal of hematopoietic stem cells. J Biol Chem, 2004. 279(24): p. 24986-93. 
108. Ronchini, C. and A.J. Capobianco, Induction of cyclin D1 transcription and CDK2 activity by Notch(ic): implication for cell cycle disruption in transformation by Notch(ic). Mol Cell Biol, 2001. 21(17): p. 5925-34.

109. Rangarajan, A., et al., Notch signaling is a direct determinant of keratinocyte growth arrest and entry into differentiation. EMBO J, 2001. 20(13): p. 3427-36.

110. Miele, L. and B. Osborne, Arbiter of differentiation and death: Notch signaling meets apoptosis. J Cell Physiol, 1999. 181(3): p. 393-409.

111. Fiuza, U.M. and A.M. Arias, Cell and molecular biology of Notch. J Endocrinol, 2007. 194(3): p. 459-74.

112. Fryer, C.J., J.B. White, and K.A. Jones, Mastermind recruits CycC:CDK8 to phosphorylate the Notch ICD and coordinate activation with turnover. Mol Cell, 2004. 16(4): p. 509-20.

113. Henrique, D., et al., Maintenance of neuroepithelial progenitor cells by Delta-Notch signalling in the embryonic chick retina. Curr Biol, 1997. 7(9): p. 661-70.

114. Radtke, F. and K. Raj, The role of Notch in tumorigenesis: oncogene or tumour suppressor? Nat Rev Cancer, 2003. 3(10): p. 756-67.

115. Kimble, J. and P. Simpson, The LIN-12/Notch signaling pathway and its regulation. Annu Rev Cell Dev Biol, 1997. 13: p. 333-61.

116. Artavanis-Tsakonas, S., K. Matsuno, and M.E. Fortini, Notch signaling. Science, 1995. 268(5208): p. 225-32.

117. Lowell, S., et al., Stimulation of human epidermal differentiation by delta-notch signalling at the boundaries of stem-cell clusters. Curr Biol, 2000. 10(9): p. 491-500.

118. Krebs, L.T., et al., Notch signaling is essential for vascular morphogenesis in mice. Genes Dev, 2000. 14(11): p. 1343-52.

119. Limbourg, F.P., et al., Essential role of endothelial Notch1 in angiogenesis. Circulation, 2005. 111(14): p. 1826-32.

120. Uyttendaele, $\mathrm{H}_{\text {., }}$ et al., Vascular patterning defects associated with expression of activated Notch4 in embryonic endothelium. Proc Natl Acad Sci U S A, 2001. 98(10): p. 5643-8.

121. Krebs, L.T., et al., Haploinsufficient lethality and formation of arteriovenous malformations in Notch pathway mutants. Genes Dev, 2004. 18(20): p. 2469-73.

122. Lawson, N.D., et al., Notch signaling is required for arterial-venous differentiation during embryonic vascular development. Development, 2001. 128(19): p. 3675-83.

123. Lawson, N.D., A.M. Vogel, and B.M. Weinstein, sonic hedgehog and vascular endothelial growth factor act upstream of the Notch pathway during arterial endothelial differentiation. Dev Cell, 2002. 3(1): p. 127-36.

124. Krebs, L.T., et al., Characterization of Notch3-deficient mice: normal embryonic development and absence of genetic interactions with a Notch1 mutation. Genesis, 2003. 37(3): p. 139-43.

125. Domenga, V., et al., Notch3 is required for arterial identity and maturation of vascular smooth muscle cells. Genes Dev, 2004. 18(22): p. 2730-5.

126. Joutel, A., et al., Notch3 mutations in CADASIL, a hereditary adult-onset condition causing stroke and dementia. Nature, 1996. 383(6602): p. 707-10.

127. Gridley, T., Notch signaling in the vasculature. Curr Top Dev Biol, 2010. 92: p. 277-309.

128. Karsan, A., The role of notch in modeling and maintaining the vasculature. Can J Physiol Pharmacol, 2005. 83(1): p. 14-23.

129. MacKenzie, F., et al., Notch4 inhibits endothelial apoptosis via RBP-Jkappa-dependent and independent pathways. J Biol Chem, 2004. 279(12): p. 11657-63.

130. Noseda, M., et al., Notch activation induces endothelial cell cycle arrest and participates in contact inhibition: role of p21Cip1 repression. Mol Cell Biol, 2004. 24(20): p. 8813-22. 
131. Liu, Z.J., et al., Regulation of Notch1 and Dll4 by vascular endothelial growth factor in arterial endothelial cells: implications for modulating arteriogenesis and angiogenesis. Mol Cell Biol, 2003. 23(1): p. 14-25.

132. Campos, A.H., et al., Determinants of Notch-3 receptor expression and signaling in vascular smooth muscle cells: implications in cell-cycle regulation. Circ Res, 2002. 91(11): p. 9991006.

133. Proweller, A., W.S. Pear, and M.S. Parmacek, Notch signaling represses myocardin-induced smooth muscle cell differentiation. J Biol Chem, 2005. 280(10): p. 8994-9004.

134. Gustafsson, M.V., et al., Hypoxia requires notch signaling to maintain the undifferentiated cell state. Dev Cell, 2005. 9(5): p. 617-28.

135. Jin, S., et al., Notch signaling regulates platelet-derived growth factor receptor-beta expression in vascular smooth muscle cells. Circ Res, 2008. 102(12): p. 1483-91.

136. Doi, H., et al., HERP1 inhibits myocardin-induced vascular smooth muscle cell differentiation by interfering with SRF binding to CArG box. Arterioscler Thromb Vasc Biol, 2005. 25(11): p. 2328-34.

137. Wang, W., et al., Coordinate Notch3-hairy-related transcription factor pathway regulation in response to arterial injury. Mediator role of platelet-derived growth factor and ERK. J Biol Chem, 2002. 277(26): p. 23165-71.

138. Lindner, V., et al., Members of the Jagged/Notch gene families are expressed in injured arteries and regulate cell phenotype via alterations in cell matrix and cell-cell interaction. Am J Pathol, 2001. 159(3): p. 875-83.

139. Li, Y., et al., Smooth muscle Notch1 mediates neointimal formation after vascular injury. Circulation, 2009. 119(20): p. 2686-92.

140. Sakata, Y., et al., Transcription factor CHF1/Hey2 regulates neointimal formation in vivo and vascular smooth muscle proliferation and migration in vitro. Arterioscler Thromb Vasc Biol, 2004. 24(11): p. 2069-74.

141. Wang, Z., Y. Li, and F.H. Sarkar, Notch signaling proteins: legitimate targets for cancer therapy. Curr Protein Pept Sci, 2010. 11(6): p. 398-408.

142. Rizzo, P., et al., Rational targeting of Notch signaling in cancer. Oncogene, 2008. 27(38): p. 5124-31.

143. Al-Hussaini, H., et al., Notch signaling pathway as a therapeutic target in breast cancer. Mol Cancer Ther, 2011. 10(1): p. 9-15.

144. Wong, G.T., et al., Chronic treatment with the gamma-secretase inhibitor $L Y-411,575$ inhibits beta-amyloid peptide production and alters lymphopoiesis and intestinal cell differentiation. J Biol Chem, 2004. 279(13): p. 12876-82.

145. Aste-Amezaga, M., et al., Characterization of Notch1 antibodies that inhibit signaling of both normal and mutated Notch1 receptors. PLoS One, 2010. 5(2): p. e9094.

146. Groth, C. and M.E. Fortini, Therapeutic approaches to modulating Notch signaling: Current challenges and future prospects. Semin Cell Dev Biol, 2012.

147. Li, X., et al., Notch3 signaling promotes the development of pulmonary arterial hypertension. Nat Med, 2009. 15(11): p. 1289-97.

148. Firth, A.L., J. Mandel, and J.X. Yuan, Idiopathic pulmonary arterial hypertension. Dis Model Mech, 2010. 3(5-6): p. 268-73.

149. Jasmin, J.F., et al., Effectiveness of a nonselective $E T(A / B)$ and a selective $E T(A)$ antagonist in rats with monocrotaline-induced pulmonary hypertension. Circulation, 2001. 103(2): p. 314-8.

150. Wilson, D.W., et al., Progressive inflammatory and structural changes in the pulmonary vasculature of monocrotaline-treated rats. Microvasc Res, 1989. 38(1): p. 57-80. 
151. Stenmark, K.R., et al., Animal models of pulmonary arterial hypertension: the hope for etiological discovery and pharmacological cure. Am J Physiol Lung Cell Mol Physiol, 2009. 297(6): p. L1013-32.

152. Mittal, M., et al., Hypoxia-dependent regulation of nonphagocytic NADPH oxidase subunit NOX4 in the pulmonary vasculature. Circ Res, 2007. 101(3): p. 258-67.

153. Schermuly, R.T., et al., Phosphodiesterase 1 upregulation in pulmonary arterial hypertension: target for reverse-remodeling therapy. Circulation, 2007. 115(17): p. 2331-9.

154. Pak, O., et al., Animal models of pulmonary hypertension: role in translational research. Drug Discovery Today: Disease Models, 2010. 7(3-4): p. 89-97.

155. Taraseviciene-Stewart, L., et al., Inhibition of the VEGF receptor 2 combined with chronic hypoxia causes cell death-dependent pulmonary endothelial cell proliferation and severe pulmonary hypertension. FASEB J, 2001. 15(2): p. 427-38.

156. Taraseviciene-Stewart, L., et al., A bradykinin antagonist and a caspase inhibitor prevent severe pulmonary hypertension in a rat model. Can J Physiol Pharmacol, 2002. 80(4): p. 269-74.

157. Iso, T., Y. Hamamori, and L. Kedes, Notch signaling in vascular development. Arterioscler Thromb Vasc Biol, 2003. 23(4): p. 543-53.

158. Hofmann, J.J. and M.L. Iruela-Arispe, Notch signaling in blood vessels: who is talking to whom about what? Circ Res, 2007. 100(11): p. 1556-68.

159. Villa, N., et al., Vascular expression of Notch pathway receptors and ligands is restricted to arterial vessels. Mech Dev, 2001. 108(1-2): p. 161-4.

160. Fung, E., et al., Delta-like 4 induces notch signaling in macrophages: implications for inflammation. Circulation, 2007. 115(23): p. 2948-56.

161. Liu, H., et al., Notch3 is critical for proper angiogenesis and mural cell investment. Circ Res, 2010. 107(7): p. 860-70.

162. Cool, C.D., et al., Three-dimensional reconstruction of pulmonary arteries in plexiform pulmonary hypertension using cell-specific markers. Evidence for a dynamic and heterogeneous process of pulmonary endothelial cell growth. Am J Pathol, 1999. 155(2): p. 411-9.

163. Jamison, B.M. and R.P. Michel, Different distribution of plexiform lesions in primary and secondary pulmonary hypertension. Hum Pathol, 1995. 26(9): p. 987-93.

164. Sakao, S., K. Tatsumi, and N.F. Voelkel, Endothelial cells and pulmonary arterial hypertension: apoptosis, proliferation, interaction and transdifferentiation. Respir Res, 2009. 10: p. 95.

165. Masumura, T., et al., Shear stress increases expression of the arterial endothelial marker ephrinB2 in murine ES cells via the VEGF-Notch signaling pathways. Arterioscler Thromb Vasc Biol, 2009. 29(12): p. 2125-31.

166. Takeshita, K., et al., Critical role of endothelial Notch1 signaling in postnatal angiogenesis. Circ Res, 2007. 100(1): p. 70-8.

167. Sweeney, C., et al., Notch 1 and 3 receptor signaling modulates vascular smooth muscle cell growth, apoptosis, and migration via a CBF-1/RBP-Jk dependent pathway. FASEB J, 2004. 18(12): p. 1421-3.

168. Lee, S.D., et al., Monoclonal endothelial cell proliferation is present in primary but not secondary pulmonary hypertension. J Clin Invest, 1998. 101(5): p. 927-34.

169. Rai, P.R., et al., The cancer paradigm of severe pulmonary arterial hypertension. Am J Respir Crit Care Med, 2008. 178(6): p. 558-64.

170. Yeager, M.E., et al., Microsatellite instability of endothelial cell growth and apoptosis genes within plexiform lesions in primary pulmonary hypertension. Circ Res, 2001. 88(1): p. E2- 
E11.

171. Masri, F.A., et al., Hyperproliferative apoptosis-resistant endothelial cells in idiopathic pulmonary arterial hypertension. Am J Physiol Lung Cell Mol Physiol, 2007. 293(3): p. L54854.

172. Weng, A.P., et al., Activating mutations of NOTCH1 in human $T$ cell acute lymphoblastic leukemia. Science, 2004. 306(5694): p. 269-71.

173. Pancewicz, J., et al., Notch signaling contributes to proliferation and tumor formation of human T-cell leukemia virus type 1-associated adult T-cell leukemia. Proc Natl Acad Sci U S A, 2010. 107(38): p. 16619-24.

174. Ranganathan, P., K.L. Weaver, and A.J. Capobianco, Notch signalling in solid tumours: a little bit of everything but not all the time. Nat Rev Cancer, 2011. 11(5): p. 338-51.

175. Liu, Z.J., et al., Inhibition of endothelial cell proliferation by Notch1 signaling is mediated by repressing MAPK and PI3K/Akt pathways and requires MAML1. FASEB J, 2006. 20(7): p. 1009-11.

176. Yang, K. and A. Proweller, Vascular smooth muscle Notch signals regulate endothelial cell sensitivity to angiogenic stimulation. J Biol Chem, 2011. 286(15): p. 13741-53.

177. LaBaer, J., et al., New functional activities for the p21 family of CDK inhibitors. Genes Dev, 1997. 11(7): p. 847-62.

178. Miyashita, T., et al., Tumor suppressor $p 53$ is a regulator of bcl-2 and bax gene expression in vitro and in vivo. Oncogene, 1994. 9(6): p. 1799-805.

179. Vaux, D.L., S. Cory, and J.M. Adams, Bcl-2 gene promotes haemopoietic cell survival and cooperates with c-myc to immortalize pre-B cells. Nature, 1988. 335(6189): p. 440-2.

180. Oltvai, Z.N., C.L. Milliman, and S.J. Korsmeyer, Bcl-2 heterodimerizes in vivo with a conserved homolog, Bax, that accelerates programmed cell death. Cell, 1993. 74(4): p. 60919.

181. Matsumoto, H., et al., Bax to BCl-2 ratio and Ki-67 index are useful predictors of neoadjuvant chemoradiation therapy in bladder cancer. Jpn J Clin Oncol, 2004. 34(3): p. 124-30.

182. Gazzaniga, P., et al., Bcl-2/bax mRNA expression ratio as prognostic factor in low-grade urinary bladder cancer. Int J Cancer, 1996. 69(2): p. 100-4.

183. Rangarajan, A., et al., Activated Notch1 signaling cooperates with papillomavirus oncogenes in transformation and generates resistance to apoptosis on matrix withdrawal through PKB/Akt. Virology, 2001. 286(1): p. 23-30.

184. Plentz, R., et al., Inhibition of gamma-secretase activity inhibits tumor progression in a mouse model of pancreatic ductal adenocarcinoma. Gastroenterology, 2009. 136(5): p. 1741-9 e6.

185. Curry, C.L., et al., Gamma secretase inhibitor blocks Notch activation and induces apoptosis in Kaposi's sarcoma tumor cells. Oncogene, 2005. 24(42): p. 6333-44.

186. Tuder, R.M., et al., Exuberant endothelial cell growth and elements of inflammation are present in plexiform lesions of pulmonary hypertension. Am J Pathol, 1994. 144(2): p. 27585.

187. Cool, C.D., et al., Pathogenesis and evolution of plexiform lesions in pulmonary hypertension associated with scleroderma and human immunodeficiency virus infection. Hum Pathol, 1997. 28(4): p. 434-42. 


\section{DECLARATION}

"I declare that I have completed this dissertation single-handedly without the unauthorized help of a second party and only with the assistance acknowledged therein. I have appropriately acknowledged and referenced all text passages that are derived literally from or are based on the content of published or unpublished work of others, and all information that relates to verbal communications. I have abided by the principles of good scientific conduct laid down in the charter of the Justus Liebig University of Giessen in carrying out the investigations described in the dissertation."

Giessen, December 2012

Swati Dabral 


\section{ACKNOWLEDGEMENTS}

First and foremost, I would like to thank my supervisor, Prof. Dr. Ralph Schermuly, for providing me with an opportunity to work in his lab and learn under his supervision. I am extremely grateful for your constant encouragement and positive attitude.

I am grateful to Prof. Dr. Dr. Thomas Braun for accepting me in the IMPRS graduate program and thus, allowing me to work in a scientifically stimulating environment. Also I would like to extend my sincere thanks to Prof. Dr. Werner Seeger for sharing his vast knowledge of pulmonary hypertension field and his valuable scientific inputs over the years.

I feel really honored to express my gratitude to, Dr. Soni S. Pullamsetti, for her scientific guidance and support. Thank you for your timely and valuable suggestions and unending patience. I owe my thanks to Dr. Rajkumar Savai, for sharing the know-how and all the helpful advises. I would also like to thank Uta Eule and Andreas Hecker for their support with animal experiments and Ewa Bieniek for the help with immunohistochemistry. Very special thanks to Anja Schmall for writing the German translation of my thesis summary.

I would specially like to express my gratitude to all my wonderful colleagues and friends. In particular, I would like to thank:

Aleksandra Tretyn, my lab mate and dear friend, for showing me around when I first came to the institute. I will always cherish our small talks over coffee breaks. Thank you for making these years fun and sharing the rollercoaster ride with me. Pavan K. Pamarthi, my closest friend during my Phd years. I had the best times with you discussing experimental troubleshootings, watching movies and playing badminton. I really miss our talks and your wise sayings.

My all other labmates, Yves Schymura, Svenja Tiede and Dr. Wiebke Janssen for making me feel an integral part of the lab. Thank you for creating a fun-working environment. Hamza Al-Tamari, for the positive spirit and all the interesting music you bring to the lab. Katharina Weidl, for help with anything, from tissue culture to planning "get togethers" for the lab.

Dr. Djuro Kosanovic, Oleg Pak and Dr. Diya Hasan, my friends, for all the nice grills, dinners and heart filled discussions. Life with you all is a big party.

Sumit, my brother, who has always been a big part of my life. Thanks for always believing in me. My husband, Abhishek, who has always been a heartbeat away. You probably know me better than anybody. Thanks for always being on my side.

Maa and Papa, my most favorite persons in this world. I would never be the person I am, without your support and belief in me. I feel really lucky to have you both. 
Der Lebenslauf wurde aus der elektronischen Version der Arbeit entfernt.

The curriculum vitae was removed from the electronic version of the paper. 


\section{Scientific Contributions}

\section{Poster Presentations}

- Dabral S, Pullamsetti SS, Ghofrani HA, Weissmann N, Grimminger F, Seeger W, Schermuly RT. "Role of Notch Signaling in Pulmonary Arterial Hypertension" Notch Meeting, Athens, Greece, October 2011

- Dabral S, Pullamsetti SS, Ghofrani HA, Weissmann N, Grimminger F, Seeger W, Schermuly RT. "Role of Notch Signaling in Pulmonary Arterial Hypertension" American Thoracic Society (ATS), Denver, Colorado, May 2011

\section{Scientic Talks}

- Role of Notch Signaling in Pulmonary Hypertension Excellence Cluster for Cardiopulmonary system (ECCPS) Retreat, Bad Nauheim, May 2010

\section{Publications}

- Pullamsetti SS, Berghausen EM, Dabral S, Tretyn A, Butrous E, Savai R, Butrous G, Dahal BK, Brandes RP, Ghofrani HA, Weissmann N, Grimminger F, Seeger W, Rosenkranz S, Schermuly RT. Role of Src tyrosine kinases in experimental pulmonary hypertension, Arterioscler Thromb Vasc Biol. 2012 Jun;32 (6):1354-65.

- Viswanath G, Chatterjee S, Dabral S, Nanguneri SR, Divya G, Roy P, Anti-androgenic endocrine disrupting activities of chlorpyrifos and piperophos, J Steroid Biochem Mol Biol. 2010 May; 120(1):22-9

\section{Activities}

- PhD students representative at Max-Planck Institute for Heart and Lung Research, Oct 2010-2011

- Coordinator and Chair for Paper Presentation Session on "Cell and Molecular Biology" at "Cognizance", IIT Technical Fest, 2007 